\title{
Gushel-Mukai varieties: Classification and birationalities
}

\author{
Olivier Debarre and Alexander Kuznetsov
}

\begin{abstract}
We perform a systematic study of Gushel-Mukai varieties - quadratic sections of linear sections of cones over the Grassmannian $\operatorname{Gr}(2,5)$. This class of varieties includes Clifford general curves of genus 6, Brill-Noether general polarized K3 surfaces of genus 6, prime Fano threefolds of genus 6, and their higher-dimensional analogues.

We establish an intrinsic characterization of normal Gushel-Mukai varieties in terms of their excess conormal sheaves, which leads to a new proof of the classification theorem of Gushel and Mukai. We give a description of isomorphism classes of Gushel-Mukai varieties and their automorphism groups in terms of linear-algebraic data naturally associated with these varieties.

We carefully develop the relation between Gushel-Mukai varieties and EisenbudPopescu-Walter (EPW) sextics introduced earlier by Iliev-Manivel and O'Grady. We describe explicitly all Gushel-Mukai varieties whose associated EPW sextics are isomorphic or dual (we call them period partners or dual varieties, respectively). Finally, we show that in dimension 3 and higher, period partners or dual varieties are always birationally isomorphic.
\end{abstract}

\section{Introduction}

This article is devoted to the investigation of the geometry of a class of varieties which we call Gushel-Mukai varieties, or GM varieties for short, which are dimensionally transverse intersections of a cone over the Grassmannian $\operatorname{Gr}(2,5)$ with a linear space and a quadratic hypersurface, all defined over a field $\mathbf{k}$ of characteristic 0 .

Smooth GM varieties are isomorphic either to quadratic sections of a linear section of $\operatorname{Gr}(2,5) \subset \mathbf{P}^{9}$ (ordinary GM varieties) or to double covers of a linear section of $\operatorname{Gr}(2,5)$ branched along a quadratic section (special GM varieties). Their dimension is at most 6 .

GM varieties first appeared in the classification of complex Fano threefolds: Gushel showed in [Gus83] that any smooth prime complex Fano threefold of degree 10 is a GM variety. Mukai later extended in [Muk89] Gushel's results to higher-dimensional smooth Fano varieties of coin-

Received 17 April 2016, accepted in final form 1 January 2017.

2010 Mathematics Subject Classification 14J45, 14E07, 14E08, 14J30, 14J35, 14J40, 14J50, $14 J 60$.

Keywords: Fano varieties, EPW sextics, Lagrangian intersection, birational isomorphisms, Gushel-Mukai varieties, excess conormal sheaves, projective duality of quadrics.

This journal is (C) Foundation Compositio Mathematica 2018. This article is distributed with Open Access under the terms of the Creative Commons Attribution Non-Commercial License, which permits non-commercial reuse, distribution, and reproduction in any medium, provided that the original work is properly cited. For commercial re-use, please contact the Foundation Compositio Mathematica.

A. K. was partially supported by the Russian Academic Excellence Project "5-100", by the RFBR grants 15-0102164 and 15-51-50045, and by the Simons foundation. 


\section{O. Debarre and A. Kuznetsov}

dex 3, degree 10, and Picard number 1, to Brill-Noether general polarized K3 surfaces of degree 10, and to Clifford general curves of genus 6 (see Section 2.3 for the definitions).

In Section 2, we give an intrinsic characterization of normal GM varieties in terms of their twisted excess conormal sheaf (Theorem 2.3). In particular, we show that the (rational) map from a GM variety to $\operatorname{Gr}(2,5)$ is given by the sections of this rank 2 sheaf, hence is canonical. Developing this observation, we define GM data sets (Definition 2.5) as sets of linear-algebraic objects required to define a GM variety. We show that there is a functorial bijection (induced by an equivalence of appropriately defined groupoids) between the set of all normal polarized GM varieties and a suitable set of GM data (Theorem 2.9). We also describe the automorphism group of a normal polarized GM variety in terms of the associated GM data (Corollary 2.11).

Using this intrinsic characterization of GM varieties, we prove an extension of Mukai's classification results (Theorem 2.16): any normal, locally factorial, complex projective variety $X$ of dimension $n \geqslant 3$ with terminal singularities and $\operatorname{codim}(\operatorname{Sing}(X)) \geqslant 4$, together with an ample Cartier divisor class $H$ such that $K_{X} \sim_{\text {lin }}-(n-2) H, H^{n}=10$, and $\operatorname{Pic}(X)=\mathbf{Z} H$, is a GM variety.

In contrast with the smooth case, a singular GM variety may have arbitrarily high dimension; besides the two smooth types discussed above (ordinary and special), there are also quadric bundles of arbitrary dimensions over a linear section of $\operatorname{Gr}(2,5)$, whose discriminant locus is a quadratic section. However, if one restricts to locally complete intersection (lci) GM varieties, one gets the ordinary and special types only. In particular, their dimension is again at most 6, and the theory becomes very much parallel to that of smooth GM varieties.

We end Section 2 by studying geometric properties of GM varieties. We define the Grassmannian hull $M_{X}$ of a GM variety $X$ as the intersection of the cone over $\operatorname{Gr}(2,5)$ in which $X$ sits with the linear span of $X$, so that $X$ is a quadratic section of $M_{X}$. We show that when $X$ is ordinary with $\operatorname{dim}(X) \geqslant 3$ and $\operatorname{codim}(\operatorname{Sing}(X)) \geqslant 4$, the scheme $M_{X}$ is smooth, and when $X$ is special with $\operatorname{codim}(\operatorname{Sing}(X)) \geqslant 4$, the scheme $M_{X}$ is a cone over a smooth variety $M_{X}^{\prime}$ (Proposition 2.22). We also discuss the singularities of $M_{X}$ for smooth ordinary GM curves and surfaces. We study the special features of lci GM varieties, we explain the relations between the two types (ordinary and special), and we construct a (birational) involution on the class of lci GM varieties which exchanges ordinary and special varieties.

In Section 3, we discuss a relation between complex GM varieties and Eisenbud-PopescuWalter (EPW) sextics [EPW01, OGr06]. An EPW sextic is a hypersurface of degree 6 in the projectivization $\mathbf{P}\left(V_{6}\right)$ of a 6 -dimensional vector space $V_{6}$ which depends on the choice of a Lagrangian subspace $A \subset \bigwedge^{3} V_{6}$. EPW sextics have many wonderful properties which were thoroughly investigated by O'Grady. A relation between GM varieties and EPW sextics was discovered by Iliev-Manivel in [IM11]. They showed that if $V_{6}$ is the space of quadrics through an ordinary 5-dimensional GM variety $X$ in the projective embedding defined by the ample generator $H$ of $\operatorname{Pic}(X)$, one can construct a Lagrangian subspace $A(X) \subset \bigwedge^{3} V_{6}$ and a hyperplane $V_{5}(X) \subset V_{6}$; conversely, given a general Lagrangian subspace $A \subset \wedge^{3} V_{6}$ and a general hyperplane $V_{5} \subset V_{6}$, one can reconstruct a 5 -dimensional GM variety $X_{A, V_{5}}$.

We suggest an extension of the Iliev-Manivel construction which works for all lci GM varieties (of both types - ordinary and special - and in all dimensions). Formally, this is done by associating with an lci GM data set another set which we call an extended Lagrangian data set. This is a quadruple $\left(V_{6}, V_{5}, A, A_{1}\right)$, where $A \subset \wedge^{3} V_{6}$ is a Lagrangian subspace and $A_{1}$, which encodes the type of the GM data, is a Lagrangian subspace in a fixed 2-dimensional symplectic space. In Theorem 3.6, we show that when the base field $\mathbf{k}$ is quadratically closed $\left(\mathbf{k}=\mathbf{k}^{1 / 2}\right)$, 


\section{Gushel-Mukai VARIETIES}

there is a bijection between the set of isomorphism classes of lci GM data sets and the set of isomorphism classes of extended Lagrangian data sets.

Concatenating this construction with the construction in Section 2, we obtain a diagram

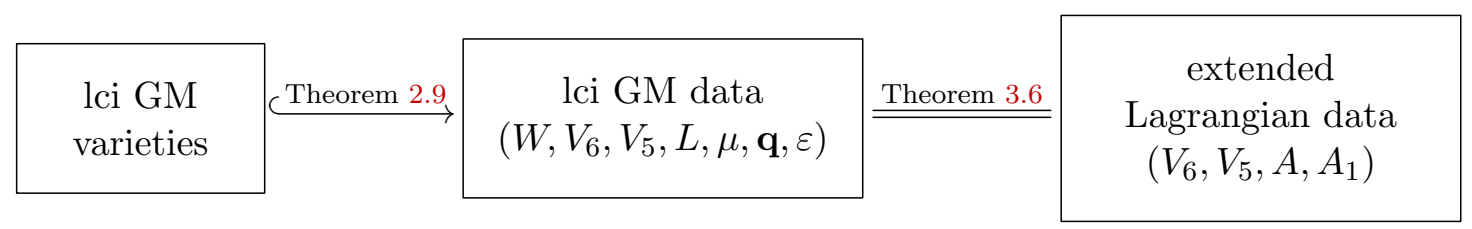

where the objects in the boxes are taken modulo isomorphisms. Restricting ourselves to ordinary GM varieties simplifies the picture: $A_{1}$ becomes redundant, so we can restrict to triples $\left(V_{6}, V_{5}, A\right)$ which we simply call Lagrangian data sets, the bijection becomes functorial and works over arbitrary fields, and the diagram above simplifies (see Theorem 3.10) to an inclusion

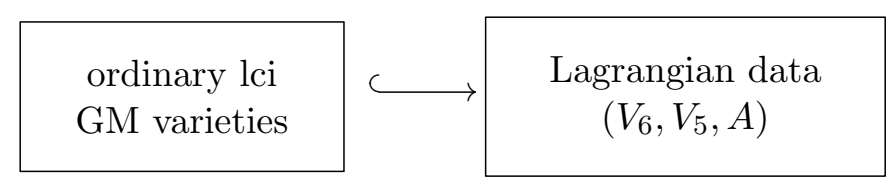

The correspondence between lci GM varieties and extended Lagrangian data sets has many remarkable properties. One of the most impressive is a criterion for the smoothness of the GM variety $X_{A, A_{1}, V_{5}}$ constructed from an extended Lagrangian data set $\left(V_{6}, V_{5}, A, A_{1}\right)$, which shows in particular that if $\operatorname{dim}\left(X_{A, A_{1}, V_{5}}\right) \geqslant 3$, smoothness is equivalent to an explicit property of the Lagrangian subspace $A \subset \bigwedge^{3} V_{6}$ (that it contains no decomposable vectors; see Section B.1) and does not depend on the hyperplane $V_{5} \subset V_{6}$ (Theorem 3.16).

We spend some time discussing the structure of lci GM varieties associated with the same Lagrangian subspace $A \subset \wedge^{3} V_{6}$, but with different choices of $A_{1}$ and hyperplane $V_{5} \subset V_{6}$. As was already mentioned, $A_{1}$ just encodes the type of the GM variety, and switching the type of $A_{1}$ results in switching the type of the GM variety. We show that for fixed $\left(A, A_{1}\right)$, the dimension of $X_{A, A_{1}, V_{5}}$ depends only on which stratum of the EPW stratification of the space $\mathbf{P}\left(V_{6}^{\vee}\right)$ the hyperplane $V_{5}$ belongs to (extending again the results of Iliev and Manivel).

If $\operatorname{dim}\left(X_{A, A_{1}, V_{5}}\right)=\operatorname{dim}\left(X_{A, A_{1}^{\prime}, V_{5}^{\prime}}\right)$, we say that the $\mathrm{GM}$ varieties $X_{A, A_{1}, V_{5}}$ and $X_{A, A_{1}^{\prime}, V_{5}^{\prime}}$ are period partners. One of the main results of this article is that smooth period partners of dimension $n \geqslant 3$ are birationally isomorphic (Theorem 4.7 and Corollary 4.16). In particular, no smooth GM varieties of dimension $n \geqslant 3$ are birationally rigid (Corollary 4.17).

We also introduce a notion of duality for GM varieties. Given a Lagrangian subspace $A$ in $\bigwedge^{3} V_{6}$, its orthogonal complement $A^{\perp} \subset \bigwedge^{3} V_{6}^{\vee}$ is also Lagrangian; the choice of a line $V_{1} \subset V_{6}$ (which can be considered as a hyperplane in $V_{6}^{\vee}$ ) and an arbitrary choice of $A_{1}^{\prime}$ allow us to construct a GM variety $X_{A^{\perp}, A_{1}^{\prime}, V_{1}}$. If $\operatorname{dim}\left(X_{A^{\perp}, A_{1}^{\prime}, V_{1}}\right)=\operatorname{dim}\left(X_{A, A_{1}, V_{5}}\right)$, we say that the GM varieties $X_{A^{\perp}, A_{1}^{\prime}, V_{1}}$ and $X_{A, A_{1}, V_{5}}$ are dual. Our second main result is that smooth dual GM varieties of dimension $n \geqslant 3$ are birationally isomorphic (Theorem 4.20).

The birational isomorphisms relating period partners are generalizations of conic transformations, and those relating dual varieties are generalizations of line transformations [DIM12]. In relation with birationalities for dual varieties, we define another interesting geometric object associated with GM varieties: a special hypersurface of degree 4 in $\operatorname{Gr}(3,6)$. Like the EPW sextic, this hypersurface is defined as a special Lagrangian intersection locus; the two play similar roles. We call the special hypersurface the EPW quartic and suspect that it may have interesting geometry. These quartics are investigated in more details in [IKKR17]. 


\section{O. Debarre and A. Kuznetsov}

For the reader's convenience, we gathered some of the material we need in the main body of this article in three appendices. In Appendix A, we introduce excess conormal sheaves for intersections of quadrics, discuss how they change under simple geometric operations (taking cones and hyperplane and quadratic sections), and compute them for the Grassmannians $\operatorname{Gr}(2,5)$ and $\operatorname{Gr}(2,6)$. In Appendix B, we recall the definition of EPW sextics and their main properties. Most of the results there are extracted from a series of articles of O'Grady. In Appendix C, we discuss the classical relation between the Lagrangian geometry of a symplectic vector space and the projective duality of quadrics. We also define a notion of isotropic reduction in this context which is very useful for the description of the various quadratic fibrations associated with Gushel-Mukai varieties.

One of the motivations for this research was an attempt to construct a moduli space for GM varieties. The unified and extended constructions we introduce here should be important ingredients to attack the moduli problem. We plan to do this in a forthcoming article.

In another, companion, article [DK16], we show that period partners of even dimensions have isomorphic primitive middle Hodge structures and describe this Hodge structure in terms of the associated EPW sextic.

In a joint work [KP16] of the second author with Alexander Perry, we discuss derived categorical aspects of GM varieties.

\subsection{Notation and conventions}

All schemes are separated of finite type over a field $\mathbf{k}$. For simplicity, we will always assume that $\mathbf{k}$ is a subfield of $\mathbf{C}$, although many of our results remain valid in positive characteristics. A kvariety is a geometrically integral scheme (separated of finite type) over $\mathbf{k}$. We usually abbreviate "local(ly) complete intersection" as "lci."

We write $\sim_{\text {lin }}$ for linear equivalence of divisors.

Given a vector space $V$, we denote by $V^{\vee}$ the dual space and, given a vector subspace $U \subset V$, we denote by $U^{\perp} \subset V^{\vee}$ its orthogonal complement, that is, the space of linear functions on $V$ vanishing on $U$.

Given a vector space $V$, we denote by $\mathbf{P}(V)$ its projectivization, that is, the variety of lines in $V$. Given a line $V_{1} \subset V$, we write $\left[V_{1}\right]$, or even $V_{1}$, for the corresponding point of $\mathbf{P}(V)$. More generally, we denote by $\operatorname{Gr}(k, V)$ the Grassmannian of $k$-dimensional subspaces in $V$ and, given a subspace $V_{k} \subset V$, we write $\left[V_{k}\right]$, or even $V_{k}$, for the corresponding point of the Grassmannian. Analogously, we denote by $\mathrm{FI}\left(k_{1}, \ldots, k_{r} ; V\right)$ the flag variety of chains of subspaces in $V$ of dimensions $k_{1}<\cdots<k_{r}$. Finally, given a vector bundle $\mathscr{E}$ on a scheme $X$, we denote by $\mathbf{P}_{X}(\mathscr{E})$ its projectivization, that is, the scheme of lines in fibers of $\mathscr{E}$ over $X$.

Let $W$ be a vector space, and let $X \subset \mathbf{P}(W)$ be a subscheme. For a point $x \in X$, we write $\mathbf{T}_{X, x}$ for the embedded tangent space of $X$ at $x$. For any vector space $K$, we denote by $\mathrm{C}_{K} X \subset \mathbf{P}(W \oplus K)$ the cone over $X$ with vertex $\mathbf{P}(K)$ and by $\mathrm{C}_{K}^{\circ} X$ the punctured cone, that is, $\mathrm{C}_{K}^{\circ} X=\mathrm{C}_{K} X \backslash \mathbf{P}(K)$.

Given closed subschemes $X_{1}, X_{2} \subset \mathbf{P}(W)$, we say that the intersection $X_{1} \cap X_{2}$ is dimensionally transverse if $\operatorname{Tor}_{>0}\left(\mathscr{O}_{X_{1}}, \mathscr{O}_{X_{2}}\right)=0$ (this condition is also known as Tor-independence). When $X_{1}$ and $X_{2}$ are both Cohen-Macaulay, this condition is equivalent to

$$
\operatorname{codim}_{x}\left(X_{1} \cap X_{2}\right)=\operatorname{codim}_{x}\left(X_{1}\right)+\operatorname{codim}_{x}\left(X_{2}\right)
$$

for any closed point $x \in X_{1} \cap X_{2}$ (where the codimensions are in $\mathbf{P}(W)$ ). When $X_{2} \subset \mathbf{P}(W)$ is 


\section{GuSHEL-MuKaI VARIETIES}

a hypersurface, the intersection $X_{1} \cap X_{2}$ is dimensionally transverse if and only if no associated point of $X_{1}$ is contained in $X_{2}$.

\section{The geometry of Gushel-Mukai varieties}

\subsection{Intrinsic characterization of GM varieties}

In the introduction, we defined a GM variety as a dimensionally transverse intersection of a cone over $\operatorname{Gr}(2,5)$ with a linear subspace and a quadratic hypersurface.

More precisely, let $V_{5}$ be a $\mathbf{k}$-vector space of dimension 5 , and consider the Plücker embedding $\operatorname{Gr}\left(2, V_{5}\right) \subset \mathbf{P}\left(\bigwedge^{2} V_{5}\right)$. Let $K$ be an arbitrary k-vector space. Consider the cone

$$
\mathrm{C}_{\mathrm{K}} \mathrm{Gr}\left(2, V_{5}\right) \subset \mathbf{P}\left(\bigwedge^{2} V_{5} \oplus K\right)
$$

with vertex $\mathbf{P}(K)$, and choose a vector subspace $W \subset \bigwedge^{2} V_{5} \oplus K$ and a subscheme $Q \subset \mathbf{P}(W)$ defined by one quadratic equation (possibly zero).

Definition 2.1. The scheme

$$
X=\mathrm{C}_{\mathrm{K}} \mathrm{Gr}\left(2, V_{5}\right) \cap \mathbf{P}(W) \cap Q
$$

is called a GM intersection. A GM intersection $X$ is a GM variety if $X$ is geometrically integral and

$$
\operatorname{dim}(X)=\operatorname{dim}(W)-5 \geqslant 1 .
$$

When $X$ is a GM variety, $Q$ is a quadratic hypersurface and $\mathrm{C}_{\mathrm{K}} \mathrm{Gr}\left(2, V_{5}\right) \cap \mathbf{P}(W)$ and $Q$ are Gorenstein subschemes of $\mathbf{P}(W)$; hence their intersection $X$ is dimensionally transverse. In particular, a GM variety is always Gorenstein.

A GM variety $X$ has a canonical polarization, the restriction $H$ of the hyperplane class on $\mathbf{P}(W)$; we will call $(X, H)$ a polarized $G M$ variety.

Definition 2.2. An isomorphism of polarized $G M$ varieties between $(X, H)$ and $\left(X^{\prime}, H^{\prime}\right)$ is a pair $(\phi, \psi)$, where $\phi: X \stackrel{\sim}{\longrightarrow} X^{\prime}$ is an isomorphism of varieties and $\psi: \mathscr{O}_{X}(H) \stackrel{\sim}{\longrightarrow} \phi^{*} \mathscr{O}_{X^{\prime}}\left(H^{\prime}\right)$ an isomorphism of line bundles. We consider the set of all polarized GM varieties as a groupoid, with this notion of isomorphism.

We denote by $\operatorname{Aut}(X, H)$ the group of automorphisms of a polarized GM variety and by $\operatorname{Aut}_{H}(X)$ its image in $\operatorname{Aut}(X)$. There is an exact sequence

$$
1 \rightarrow \mathbb{G}_{\mathrm{m}} \rightarrow \operatorname{Aut}(X, H) \rightarrow \operatorname{Aut}_{H}(X) \rightarrow 1,
$$

where the subgroup $\mathbb{G}_{\mathrm{m}} \subset \operatorname{Aut}(X, H)$ acts trivially on $X$ and by dilations on $\mathscr{O}_{X}(H)$.

The definition of a GM variety is not intrinsic. The following theorem gives an intrinsic characterization, at least for normal varieties (note that a GM variety is normal as soon as it is non-singular in codimension 1). A key ingredient is the excess conormal sheaf, which is defined over $\mathbf{k}$, for any variety which is an intersection of quadrics (see Appendix A).

Recall that a coherent sheaf $\mathscr{F}$ is $\operatorname{simple}$ if $\operatorname{Hom}(\mathscr{F}, \mathscr{F})=\mathbf{k}$.

Theorem 2.3. A normal polarized projective variety $(X, H)$ of dimension $n \geqslant 1$ is a polarized $G M$ variety if and only if all the following conditions hold:

(a) We have $H^{n}=10$ and $K_{X}=-(n-2) H$; in particular, $X$ is Gorenstein. 


\section{O. Debarre And A. KuZnetsov}

(b) The polarization $H$ is very ample and the vector space

$$
W:=H^{0}\left(X, \mathscr{O}_{X}(H)\right)^{\vee}
$$

has dimension $n+5$.

(c) The variety $X$ is an intersection of quadrics in $\mathbf{P}(W)$ and the vector space

$$
V_{6}:=H^{0}\left(\mathbf{P}(W), \mathscr{I}_{X}(2)\right) \subset \mathrm{S}^{2} W^{\vee}
$$

of quadrics through $X$ has dimension 6 .

(d) The twisted excess conormal sheaf $\mathscr{U}_{X}:=\mathscr{E}_{N}^{\vee}{ }_{X}^{\vee}(2 H)$ of $X$ in $\mathbf{P}(W)$ is simple.

Proof. We first prove that conditions (a)-(d) are satisfied by normal GM varieties. Let $X$ be such a variety, defined by $(2.1)$, with $\operatorname{dim}(X)=n$ and $\operatorname{dim}(W)=n+5$.

(a) Since the degree of $\mathrm{C}_{\mathrm{K}} \mathrm{Gr}\left(2, V_{5}\right)$ is 5 and the degree of $Q$ is 2, the dimensional transversality implies that the degree of $X$ is 10 . Let $\operatorname{dim}(K)=k$. The canonical class of $\mathrm{C}_{\mathrm{K}} \mathrm{Gr}\left(2, V_{5}\right)$ is $-(5+k) H$. On the other hand, the codimension of $W$ in $\bigwedge^{2} V_{5} \oplus K$ is $(10+k)-(n+5)=5+k-n$; hence the canonical class of $X$ is

$$
K_{X}=(-(5+k)+(5+k-n)+2) H=-(n-2) H .
$$

(b) The very ampleness of $H$ is clear. By (2.2), it is enough to show $H^{0}\left(X, \mathscr{O}_{X}(H)\right)=W^{\vee}$. We use the resolution

$$
0 \rightarrow \mathscr{O}(-5) \rightarrow V_{5}^{\vee} \otimes \mathscr{O}(-3) \rightarrow V_{5} \otimes \mathscr{O}(-2) \rightarrow \mathscr{O} \rightarrow \mathscr{O}_{\mathrm{CK}_{\mathrm{K}} \mathrm{Gr}\left(2, V_{5}\right)} \rightarrow 0
$$

of the cone $C_{K} \operatorname{Gr}\left(2, V_{5}\right)$ in $\mathbf{P}\left(\bigwedge^{2} V_{5} \oplus K\right)$. Restricting it to $\mathbf{P}(W)$, tensoring with the resolution $0 \rightarrow \mathscr{O}(-2) \rightarrow \mathscr{O} \rightarrow \mathscr{O}_{Q} \rightarrow 0$ of the quadric $Q$, and using the dimensional transversality of the intersection, we obtain the resolution

$$
\begin{aligned}
0 & \rightarrow \mathscr{O}(-7) \rightarrow\left(V_{5}^{\vee} \oplus \mathbf{k}\right) \otimes \mathscr{O}(-5) \rightarrow\left(V_{5} \otimes \mathscr{O}(-4)\right) \oplus\left(V_{5}^{\vee} \otimes \mathscr{O}(-3)\right) \\
& \rightarrow\left(V_{5} \oplus \mathbf{k}\right) \otimes \mathscr{O}(-2) \rightarrow \mathscr{O} \rightarrow \mathscr{O}_{X} \rightarrow 0
\end{aligned}
$$

of $X$ in $\mathbf{P}(W)$. Twisting it by $\mathscr{O}(1)$, we get $H^{0}\left(X, \mathscr{O}_{X}(H)\right)=H^{0}\left(\mathbf{P}(W), \mathscr{O}_{\mathbf{P}(W)}(1)\right)=W^{\vee}$ (since $\operatorname{dim}(W)=n+5 \geqslant 6$, the only other term that could contribute is the term $\mathscr{O}(-6)$ at the very beginning; the contribution is non-zero only for $n=1$, but it actually contributes to $\left.H^{1}\left(X, \mathscr{O}_{X}(H)\right)\right)$.

(c) The resolution (2.4) implies that $X$ is an intersection of quadrics. Furthermore, twisting the resolution by $\mathscr{O}(2)$, we see that $H^{0}\left(\mathbf{P}(W), \mathscr{I}_{X}(2 H)\right)=V_{5} \oplus \mathbf{k}$ is 6-dimensional.

(d) To prove that $\mathscr{U}_{X}$ is simple, we may assume $\mathbf{k}=\mathbf{C}$.

Assume $n=1$. By condition (a), we have $\operatorname{deg}\left(K_{X}\right)=10$, so $X$ is a smooth curve of genus 6 . Moreover, by conditions (b) and (c), its canonical class $K_{X}=H$ is very ample and its canonical model is an intersection of quadrics; hence $X$ is not hyperelliptic, nor trigonal, nor a plane quintic. Mukai constructs in [Muk93, Section 5] a stable vector bundle of rank 2 on $X$ and proves that it embeds $X$ into the cone $\operatorname{CGr}(2,5)$ over $\operatorname{Gr}(2,5)$ with vertex a point and that $X$ is an intersection $\operatorname{CGr}(2,5) \cap \mathbf{P}^{5} \cap Q$. A combination of Proposition A.7 and Lemmas A.4, A.5 and A.6 then shows that Mukai's bundle is isomorphic to the twisted excess conormal bundle $\mathscr{U}_{X}$, which is therefore stable, hence simple.

We finish the proof by induction on $n$ : assume $n \geqslant 2$, and let $X^{\prime} \subset X$ be a dimensionally transverse, normal hyperplane section of $X$, so that $X^{\prime}$ is a GM variety of dimension $n-1$ and $\mathscr{U}_{X^{\prime}}$ is simple by the induction hypothesis. Applying the functor $\operatorname{Hom}\left(\mathscr{U}_{X},-\right)$ to the exact 


\section{Gushel-Mukai VARIETIES}

sequence

$$
\left.0 \rightarrow \mathscr{U}_{X}(-H) \rightarrow \mathscr{U}_{X} \rightarrow \mathscr{U}_{X}\right|_{X^{\prime}} \rightarrow 0
$$

and using pullback-pushforward adjunction and the isomorphism $\left.\mathscr{U}_{X^{\prime}} \simeq \mathscr{U}_{X}\right|_{X^{\prime}}$ (Lemma A.5), we obtain an exact sequence

$$
0 \rightarrow \operatorname{Hom}\left(\mathscr{U}_{X}, \mathscr{U}_{X}(-H)\right) \rightarrow \operatorname{Hom}\left(\mathscr{U}_{X}, \mathscr{U}_{X}\right) \rightarrow \operatorname{Hom}\left(\mathscr{U}_{X^{\prime}}, \mathscr{U}_{X^{\prime}}\right)
$$

If $\operatorname{dim}\left(\operatorname{Hom}\left(\mathscr{U}_{X}, \mathscr{U}_{X}\right)\right)>1$, the simplicity of $\mathscr{U}_{X^{\prime}}$ implies $\operatorname{Hom}\left(\mathscr{U}_{X}, \mathscr{U}_{X}(-H)\right) \neq 0$. On the other hand, an analogous argument produces an exact sequence

$$
0 \rightarrow \operatorname{Hom}\left(\mathscr{U}_{X}, \mathscr{U}_{X}(-2 H)\right) \rightarrow \operatorname{Hom}\left(\mathscr{U}_{X}, \mathscr{U}_{X}(-H)\right) \rightarrow \operatorname{Hom}\left(\mathscr{U}_{X^{\prime}}, \mathscr{U}_{X^{\prime}}(-H)\right)=0,
$$

which implies $\operatorname{Hom}\left(\mathscr{U}_{X}, \mathscr{U}_{X}(-2 H)\right) \neq 0$. Iterating the argument, we see that for all $k \geqslant 0$, we have $\operatorname{Hom}\left(\mathscr{U}_{X}, \mathscr{U}_{X}(-k H)\right) \neq 0$. This is possible only if $\mathscr{U}_{X}$ has zero-dimensional torsion. But it has not since, by (A.1), it is a subsheaf of $V_{6} \otimes \mathscr{O}_{X}$ and $X$ is integral.

We now prove that conditions (a)-(d) are also sufficient.

Let $X$ be a normal projective variety with a Cartier divisor $H$ which satisfies properties (a)-(d) of Theorem 2.3. Consider the spaces $W$ and $V_{6}$ of respective dimensions $n+5$ and 6 , defined by conditions (b) and (c). Since $V_{6}$ is a space of quadrics in $W$, it comes with a map

$$
\mathbf{q}: V_{6} \rightarrow \mathrm{S}^{2} W^{\vee}
$$

Consider the twisted excess conormal sheaf $\mathscr{U}_{X}$. Its restriction $\mathscr{U}_{X_{\mathrm{sm}}}$ to the smooth locus $X_{\mathrm{sm}}$ of $X$ is locally free of rank 2. Since the rank of the conormal sheaf $\mathscr{N}_{X_{\mathrm{sm}} / \mathbf{P}(W)}^{\vee}$ is 4, we compute, using the exact sequence (A.1),

$$
\begin{aligned}
\operatorname{det}\left(\mathscr{U}_{X_{\mathrm{sm}}}\right) & \simeq \operatorname{det}\left(\mathscr{N}_{X_{\mathrm{sm}} / \mathbf{P}(W)}(-2 H)\right) \simeq \operatorname{det}\left(\mathscr{N}_{X_{\mathrm{sm}} / \mathbf{P}(W)}\right)(-8 H) \\
& \simeq \mathscr{O}_{X_{\mathrm{sm}}}\left(-8 H-\left.K_{\mathbf{P}(W)}\right|_{X_{\mathrm{sm}}}+K_{X_{\mathrm{sm}}}\right) \simeq \mathscr{O}_{X_{\mathrm{sm}}}(-8 H+(n+5) H-(n-2) H) \\
& \simeq \mathscr{O}_{X_{\mathrm{sm}}}(-H) .
\end{aligned}
$$

Set $L:=H^{0}\left(X_{\mathrm{sm}}, \wedge^{2} \mathscr{U}_{X_{\mathrm{sm}}}(H)\right)$; by the above isomorphism and the normality of $X$, this vector space is 1-dimensional, and we have a canonical isomorphism

$$
L \otimes \bigwedge^{2} \mathscr{U}_{X_{\mathrm{sm}}}^{\vee}=\mathscr{O}_{X_{\mathrm{sm}}}(H) .
$$

Dualizing the embedding $\mathscr{U}_{X_{\mathrm{sm}}} \hookrightarrow V_{6} \otimes \mathscr{O}_{X_{\mathrm{sm}}}$ and taking its wedge square, we get a surjection $\bigwedge^{2} V_{6}^{\vee} \otimes \mathscr{O}_{X_{\mathrm{sm}}} \rightarrow \bigwedge^{2} \mathscr{U}_{X_{\mathrm{sm}}}^{\vee}$. Taking into account (2.5), we obtain a linear map

$$
L \otimes \bigwedge^{2} V_{6}^{\vee} \rightarrow H^{0}\left(X_{\mathrm{sm}}, \mathscr{O}_{X_{\mathrm{sm}}}(H)\right)=H^{0}\left(X, \mathscr{O}_{X}(H)\right)=W^{\vee}
$$

(we again use the normality of $X$ to identify sections on $X$ and on $X_{\mathrm{sm}}$ ) and by duality a linear map $\mu: L \otimes W \rightarrow \bigwedge^{2} V_{6}$. This map can be factored through an injection $\bar{\mu}: L \otimes W \hookrightarrow \bigwedge^{2} V_{6} \oplus K$ for some vector space $K$. The maps

$$
X_{\mathrm{sm}} \hookrightarrow \mathbf{P}(W) \simeq \mathbf{P}(L \otimes W) \hookrightarrow \mathbf{P}\left(\bigwedge^{2} V_{6} \oplus K\right)-\rightarrow \mathbf{P}\left(\bigwedge^{2} V_{6}\right)
$$

and

$$
X_{\mathrm{sm}} \rightarrow \operatorname{Gr}\left(2, V_{6}\right) \hookrightarrow \mathbf{P}\left(\bigwedge^{2} V_{6}\right),
$$

where the map $X_{\mathrm{sm}} \rightarrow \operatorname{Gr}\left(2, V_{6}\right)$ is induced by the embedding $\mathscr{U}_{X_{\mathrm{sm}}} \hookrightarrow V_{6} \otimes \mathscr{O}_{X_{\mathrm{sm}}}$, are given by the same linear system; hence they agree. This shows $X \subset \mathrm{C}_{K} \operatorname{Gr}\left(2, V_{6}\right)$ and $X_{\mathrm{sm}} \subset \mathrm{C}_{K}^{\circ} \mathrm{Gr}\left(2, V_{6}\right)$, 


\section{O. Debarre and A. Kuznetsov}

so by Proposition A.3, we have a commutative diagram

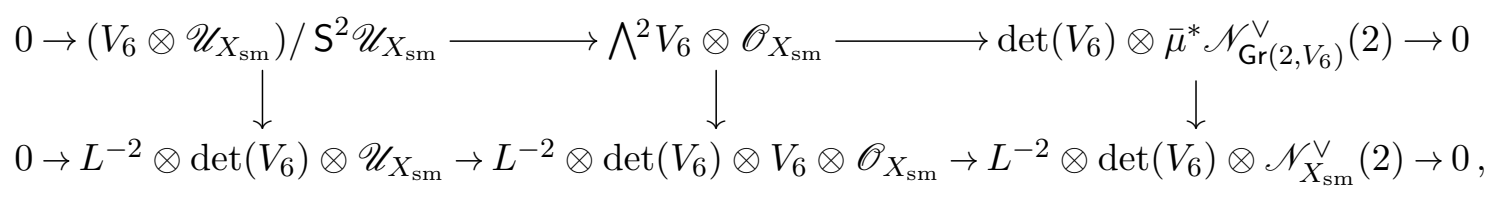

where the top row comes from the pullback to $X_{\mathrm{sm}}$ of the excess conormal sequence of $\operatorname{Gr}\left(2, V_{6}\right)$ (see Proposition A.8) and we twisted everything by $\operatorname{det}\left(V_{6}\right)$. The left vertical arrow induces a morphism $\lambda^{\prime}: V_{6} \otimes \mathscr{U}_{X_{\mathrm{sm}}} \rightarrow L^{-2} \otimes \operatorname{det}\left(V_{6}\right) \otimes \mathscr{U}_{X_{\mathrm{sm}}}$.

By condition (d), the sheaf $\mathscr{U}_{X}$ is simple. Therefore $\mathscr{U}_{X_{\mathrm{sm}}}$ is simple as well: the sheaf $\mathscr{U}_{X}$ is torsion free; hence any endomorphism of $\mathscr{U}_{X_{\mathrm{sm}}}$ extends to an endomorphism of $\mathscr{U}_{X}$. Therefore, $\lambda^{\prime}$ is given by a linear form

$$
\lambda: V_{6} \rightarrow L^{-2} \otimes \operatorname{det}\left(V_{6}\right) .
$$

Since $\lambda^{\prime}$ vanishes on $S^{2} \mathscr{U}_{X_{\mathrm{sm}}}$, the image of $\mathscr{U}_{X_{\mathrm{sm}}}$ in $V_{6} \otimes \mathscr{O}_{X_{\mathrm{sm}}}$ (via the sequence (A.1)) is contained in $\operatorname{Ker}(\lambda) \otimes \mathscr{O}_{X_{\mathrm{sm}}}$. Moreover, the middle vertical map in the commutative diagram above is given by $v_{1} \wedge v_{2} \mapsto \lambda\left(v_{1}\right) v_{2}-\lambda\left(v_{2}\right) v_{1}$.

Let us show that the form $\lambda$ is non-zero. If $\lambda=0$, the middle vertical map in the diagram is zero, which means that all the quadrics cutting out $C_{K} \operatorname{Gr}\left(2, V_{6}\right)$ contain $\mathbf{P}(W)$; in other words, $\mathbf{P}(W) \subset \mathrm{C}_{K} \operatorname{Gr}\left(2, V_{6}\right)$. Therefore, $\mathbf{P}(W)$ is a cone over $\mathbf{P}\left(W^{\prime}\right) \subset \operatorname{Gr}\left(2, V_{6}\right)$, with vertex a subspace of $K$. The map $X_{\mathrm{sm}} \rightarrow \operatorname{Gr}\left(2, V_{6}\right)$ induced by $\mathscr{U}_{X_{\mathrm{sm}}}$ therefore factors through $\mathbf{P}\left(W^{\prime}\right)$; that is, the vector bundle $\mathscr{U}_{X_{\mathrm{sm}}}$ is a pullback from $\mathbf{P}\left(W^{\prime}\right)$ of the restriction of the tautological bundle of $\operatorname{Gr}\left(2, V_{6}\right)$ to $\mathbf{P}\left(W^{\prime}\right)$. We show that this is impossible.

There are two types of linear spaces on $\operatorname{Gr}\left(2, V_{6}\right)$ : the first type corresponds to 2-dimensional subspaces containing a given vector and the second type to those contained in a given 3-subspace $V_{3} \subset V_{6}$. If $W^{\prime}$ is of the first type, the restriction of the tautological bundle to $\mathbf{P}\left(W^{\prime}\right)$ is isomorphic to $\mathscr{O} \oplus \mathscr{O}(-1)$; hence $\mathscr{U}_{X_{\mathrm{sm}}} \simeq \mathscr{O}_{X_{\mathrm{sm}}} \oplus \mathscr{O}_{X_{\mathrm{sm}}}(-H)$. In particular, it is not simple, which gives a contradiction. If $W^{\prime}$ is of the second type, the embedding $\mathscr{U}_{X} \rightarrow V_{6} \otimes \mathscr{O}_{X}$ factors through a subbundle $V_{3} \otimes \mathscr{O}_{X} \subset V_{6} \otimes \mathscr{O}_{X}$. Recall that $V_{6}$ is the space of quadrics passing through $X$ in $\mathbf{P}(W)$. Consider the scheme-theoretic intersection $M$ of the quadrics corresponding to the vector subspace $V_{3} \subset V_{6}$. Since the embedding of the excess conormal sheaf factors through $V_{3} \otimes \mathscr{O}_{X}$, the variety $X$ is the complete intersection of $M$ with the three quadrics corresponding to the quotient space $V_{6} / V_{3}$. But the degree of $X$ is then divisible by 8 , which contradicts the fact that it is 10 by condition (a).

Thus $\lambda \neq 0$ and $V_{5}:=\operatorname{Ker}(\lambda)$ is a hyperplane in $V_{6}$. It fits in the exact sequence

$$
0 \rightarrow V_{5} \rightarrow V_{6} \stackrel{\lambda}{\longrightarrow} L^{-2} \otimes \operatorname{det}\left(V_{6}\right) \rightarrow 0
$$

which induces a canonical isomorphism

$$
\varepsilon: \operatorname{det}\left(V_{5}\right) \stackrel{\sim}{\longrightarrow} L^{\otimes 2} .
$$

Moreover, the composition $\mathscr{U}_{X} \hookrightarrow V_{6} \otimes \mathscr{O}_{X} \stackrel{\lambda}{\longrightarrow} L^{\otimes 2} \otimes \operatorname{det}\left(V_{6}\right) \otimes \mathscr{O}_{X}$ vanishes on $X_{\mathrm{sm}}$, hence on $X$ as well. This shows that the embedding $\mathscr{U}_{X} \hookrightarrow V_{6} \otimes \mathscr{O}_{X}$ factors through $V_{5} \otimes \mathscr{O}_{X}$.

We now replace $V_{6}$ with $V_{5}$ and repeat the above argument. We get a linear map

$$
\mu: L \otimes W \rightarrow \bigwedge^{2} V_{5}
$$

and an embedding $\bar{\mu}: L \otimes W \hookrightarrow \bigwedge^{2} V_{5} \oplus K$ which induce embeddings $X \subset C_{K} \operatorname{Gr}\left(2, V_{5}\right)$ and 


\section{GuSHEL-MuKaI VARIETIES}

$X_{\mathrm{sm}} \subset \mathrm{C}_{K}^{\circ} \mathrm{Gr}\left(2, V_{5}\right)$. The commutative diagram

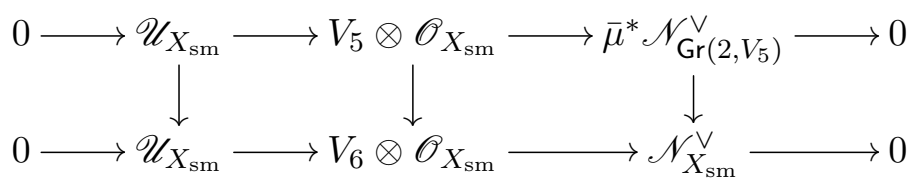

of Proposition A.3 (we use $\varepsilon$ to cancel out the twists by $\operatorname{det}\left(V_{5}^{\vee}\right)$ and by $L^{2}$ in the top and bottom rows) then shows that inside the space $V_{6}$ of quadrics cutting out $X$ in $\mathbf{P}(W)$, the hyperplane $V_{5}$ is the space of quadratic equations of $\operatorname{Gr}\left(2, V_{5}\right)$, that is, of Plücker quadrics.

As the Plücker quadrics cut out the cone $C_{K} G r\left(2, V_{5}\right)$ in $\mathbf{P}\left(\bigwedge^{2} V_{5} \oplus K\right)$, they cut out the intersection $\mathrm{C}_{\mathrm{K}} \operatorname{Gr}\left(2, V_{5}\right) \cap \mathbf{P}(W)$ in $\mathbf{P}(W)$. Since $X$ is the intersection of six quadrics by condition (c), we finally obtain

$$
X=\mathrm{C}_{\mathrm{K}} \operatorname{Gr}\left(2, V_{5}\right) \cap \mathbf{P}(W) \cap Q,
$$

where $Q$ is any non-Plücker quadric (corresponding to a point in $V_{6} \backslash V_{5}$ ), so $X$ is a GM variety.

A consequence of Theorem 2.3 is that being a GM variety is a geometric property. Recall that the base field $\mathbf{k}$ is by assumption a subfield of $\mathbf{C}$. We denote by $X_{\mathbf{C}}$ the extension of scalars from $\mathbf{k}$ to $\mathbf{C}$, and by $H_{\mathbf{C}}$ the induced polarization of $X_{\mathbf{C}}$.

Corollary 2.4. A normal polarized variety $(X, H)$ is a polarized $G M$ variety if and only if $\left(X_{\mathbf{C}}, H_{\mathbf{C}}\right)$ is.

Proof. One direction is clear from the definition, so we only have to check that if $\left(X_{\mathbf{C}}, H_{\mathbf{C}}\right)$ is $\mathrm{GM}$, so is $(X, H)$. We show that if the conditions of Theorem 2.3 are satisfied for $\left(X_{\mathbf{C}}, H_{\mathbf{C}}\right)$, they are satisfied for $(X, H)$ as well. The only property for which this is not completely obvious is the equality $K_{X}=-(n-2) H$, but that follows from the injectivity of the map $\operatorname{Pic}(X) \rightarrow \operatorname{Pic}\left(X_{\mathbf{C}}\right)$, which holds since $X$ is integral and projective.

We introduce some more terminology. Given a GM variety $X$ defined by (2.1), the twisted excess conormal sheaf $\mathscr{U}_{X}$ that was crucial for the proof of Theorem 2.3 will be called its Gushel sheaf. As we showed in the proof, the projection of $X$ from the vertex $\mathbf{P}(K)$ of the cone $C_{K} \operatorname{Gr}\left(2, V_{5}\right)$ defines a rational map $X \rightarrow \operatorname{Gr}\left(2, V_{5}\right)$, and the Gushel sheaf $\mathscr{U}_{X}$ is isomorphic (on the smooth locus of $X$ ) to the pullback under this map of the tautological vector bundle on $\operatorname{Gr}\left(2, V_{5}\right)$. The map $X \rightarrow \operatorname{Gr}\left(2, V_{5}\right)$ is thus determined by $\mathscr{U}_{X}$ and is canonically associated with $X$. We call this map the Gushel map of $X$.

\subsection{GM data sets}

In the course of the proof of Theorem 2.3, we associated with any normal polarized GM variety vector spaces $W, V_{6}, V_{5}, L$ and maps $\mathbf{q}, \mu, \varepsilon$. We axiomatize these as follows.

Definition 2.5. A $G M$ data set $\left(W, V_{6}, V_{5}, L, \mu, \mathbf{q}, \varepsilon\right)$ of dimension $n$ (over $\mathbf{k}$ ) consists of

- a k-vector space $W$ of dimension $n+5$,

- a k-vector space $V_{6}$ of dimension 6 ,

- a k-hyperplane $V_{5} \subset V_{6}$,

- a k-vector space $L$ of dimension 1 ,

- a k-linear map $\mu: L \otimes W \rightarrow \bigwedge^{2} V_{5}$, 


\section{O. Debarre And A. KuZnetsov}

- a k-linear map q: $V_{6} \rightarrow \mathrm{S}^{2} W^{\vee}$,

- a k-linear isomorphism $\varepsilon$ : $\operatorname{det}\left(V_{5}\right) \stackrel{\sim}{\rightarrow} L^{\otimes 2}$,

such that the following diagram commutes:

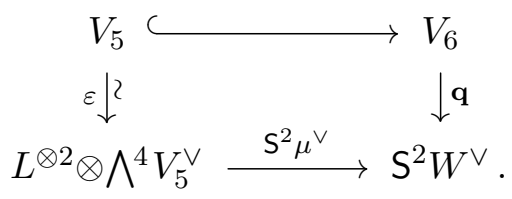

In other words, $\mathbf{q}(v)\left(w_{1}, w_{2}\right)=\varepsilon\left(v \wedge \mu\left(w_{1}\right) \wedge \mu\left(w_{2}\right)\right)$ for all $v \in V_{5}$ and $w_{1}, w_{2} \in W$.

Definition 2.6. An isomorphism of $G M$ data sets between GM data sets $\left(W, V_{6}, V_{5}, L, \mu, \mathbf{q}, \varepsilon\right)$ and $\left(W^{\prime}, V_{6}^{\prime}, V_{5}^{\prime}, L^{\prime}, \mu^{\prime}, \mathbf{q}^{\prime}, \varepsilon^{\prime}\right)$ is a triple of k-linear isomorphisms $\varphi_{W}: W \rightarrow W^{\prime}, \varphi_{V}: V_{6} \rightarrow V_{6}^{\prime}$, and $\varphi_{L}: L \rightarrow L^{\prime}$, such that

$$
\varphi_{V}\left(V_{5}\right)=V_{5}^{\prime}, \quad \varepsilon^{\prime} \circ \bigwedge^{5} \varphi_{V}=\varphi_{L}^{\otimes 2} \circ \varepsilon,
$$

and the following diagrams commute:
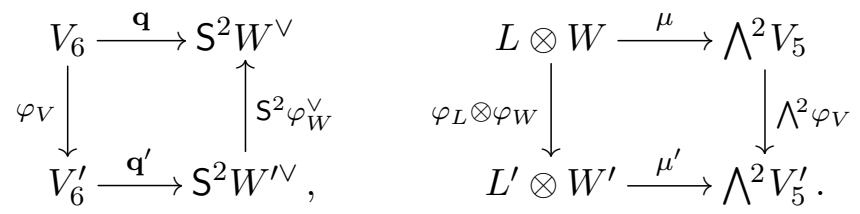

In particular, the automorphism group of a GM data set $\left(W, V_{6}, V_{5}, L, \mu, \mathbf{q}, \varepsilon\right)$ is the subgroup of $\mathrm{GL}(W) \times \mathrm{GL}\left(V_{6}\right) \times \mathbb{G}_{\mathrm{m}}$ of elements $\left(g_{W}, g_{V}, g_{L}\right)$ such that

$$
g_{V}\left(V_{5}\right)=V_{5}, \quad \operatorname{det}\left(\left.g_{V}\right|_{V_{5}}\right)=g_{L}^{2}, \quad\left(\mathrm{~S}^{2} g_{W}^{\vee}\right) \circ \mathbf{q} \circ g_{V}=\mathbf{q}, \quad\left(\bigwedge^{2} g_{V}\right) \circ \mu=\mu \circ\left(g_{L} \otimes g_{W}\right) .
$$

We consider the set of all polarized GM data sets as a groupoid, with this notion of isomorphism.

Lemma 2.7. The collection $\left(W, V_{6}, V_{5}, L, \mu, \mathbf{q}, \varepsilon\right)$ associated by Theorem 2.3 with a normal polarized $G M$ variety $(X, H)$ is a $G M$ data set. The association

$$
(X, H) \longmapsto\left(W, V_{6}, V_{5}, L, \mu, \mathbf{q}, \varepsilon\right)
$$

is a fully faithful functor from the groupoid of normal polarized GM varieties to the groupoid of GM data sets; that is, any isomorphism of polarized GM varieties induces an isomorphism of the associated GM data sets and vice versa. Moreover, this association works in families.

Proof. In the course of the proof of Theorem 2.3, we showed that the subspace $V_{5} \subset V_{6}$ of quadrics through $X$ cuts out $\mathrm{C}_{\mathrm{K}} \mathrm{Gr}\left(2, V_{5}\right) \cap \mathbf{P}(W)$ in $\mathbf{P}(W)$. Thus, these quadrics are restrictions to $\mathbf{P}(W)$ of the Plücker quadrics. This is equivalent to the commutativity of (2.7), so the constructed data forms a GM data set.

Let $(\phi, \psi)$ be an isomorphism of polarized GM varieties between $(X, H)$ and $\left(X^{\prime}, H^{\prime}\right)$. We denote by $\left(W^{\prime}, V_{6}^{\prime}, V_{5}^{\prime}, L^{\prime}, \mu^{\prime}, \mathbf{q}^{\prime}, \varepsilon^{\prime}\right)$ the GM data for $X^{\prime}$. The isomorphism $(\phi, \psi)$ induces an isomorphism between $W$ and $W^{\prime}$, and also between $V_{6}$ and $V_{6}^{\prime}$. Moreover, it induces an isomorphism between the excess conormal sheaves of $X$ and $X^{\prime}$, and an isomorphism between $L$ and $L^{\prime}$, compatible with the isomorphisms (2.5). These isomorphisms are compatible with the hyperplanes $V_{5}$ and with the maps $\mathbf{q}, \mu$, and $\varepsilon$, hence provide an isomorphism of the associated GM data sets. Moreover, the composition of isomorphisms of polarized GM varieties corresponds to the composition of the corresponding isomorphisms of GM data sets. This proves the functoriality. 


\section{Gushel-Mukai VARIETIES}

Conversely, an isomorphism of GM data sets includes an isomorphism $\varphi_{W}: \mathbf{P}(W) \rightarrow \mathbf{P}\left(W^{\prime}\right)$ which induces an isomorphism of polarized GM varieties between $X$ and $X^{\prime}$. This proves that the functor is fully faithful.

Finally, given a flat family $\mathscr{X} \rightarrow S$ of normal projective varieties with a Cartier divisor $H$ on $\mathscr{X}$ such that each fiber of the family satisfies the conditions of Theorem 2.3, the same construction provides vector bundles $\mathscr{W}, \mathscr{V}_{6}, \mathscr{V}_{5}$, and $\mathscr{L}$ on $S$ (in the definition of $\mathscr{V}_{6}, \mathscr{W}$, and $\mathscr{L}$, one should replace global sections with pushforwards to $S$ ) and morphisms q: $\mathscr{V}_{6} \rightarrow \mathrm{S}^{2} \mathscr{W}^{\vee}$, $\mu: \mathscr{L} \otimes \mathscr{W} \rightarrow \bigwedge^{2} \mathscr{V}_{5}$, and $\varepsilon: \operatorname{det}\left(\mathscr{V}_{5}\right) \stackrel{\sim}{\rightarrow} \mathscr{L}^{\otimes 2}$. This shows that the association works in families.

The following lemma characterizes the image of the functor. Let $\left(W, V_{6}, V_{5}, L, \mu, \mathbf{q}, \varepsilon\right)$ be a GM data set. For each vector $v \in V_{6}$, we have a quadratic form $\mathbf{q}(v) \in \mathrm{S}^{2} W^{\vee}$, and we denote by $Q(v) \subset \mathbf{P}(W)$ the subscheme it defines (a quadratic hypersurface when $\mathbf{q}(v) \neq 0$ ).

LEMma 2.8. If $\left(W, V_{6}, V_{5}, L, \mu, \mathbf{q}, \varepsilon\right)$ is a $G M$ data set of dimension $n$,

$$
X=X\left(W, V_{6}, V_{5}, L, \mu, \mathbf{q}, \varepsilon\right):=\bigcap_{v \in V_{6}} Q(v) \subset \mathbf{P}(W)
$$

is a GM intersection of dimension at least $n$. It is a GM variety if and only if $X$ is integral of dimension $n$.

Proof. Lift the map $\mu: L \otimes W \rightarrow \bigwedge^{2} V_{5}$ to an embedding $\bar{\mu}: L \otimes W \hookrightarrow \bigwedge^{2} V_{5} \oplus K$ for some $K$ (for example, one can take $K=\operatorname{Ker}(\mu))$. It follows from the commutativity of (2.7) that for $v \in V_{5}$, the quadrics $Q(v)$ are the restrictions to $\mathbf{P}(W)=\mathbf{P}(L \otimes W)$ of the cones (with vertex $\mathbf{P}(K)$ ) over the Plücker quadrics in $\mathbf{P}\left(\bigwedge^{2} V_{5}\right)$ with respect to the embedding $\bar{\mu}$. Since the intersection of the Plücker quadrics is $\operatorname{Gr}\left(2, V_{5}\right)$, this implies

$$
\bigcap_{v \in V_{5}} Q(v)=\mathrm{C}_{\mathrm{K}} \operatorname{Gr}\left(2, V_{5}\right) \cap \mathbf{P}(W)
$$

and, for any $v \in V_{6} \backslash V_{5}$, we have

$$
X=\mathrm{C}_{\mathrm{K}} \mathrm{Gr}\left(2, V_{5}\right) \cap \mathbf{P}(W) \cap Q(v) .
$$

This is a GM intersection, which is a GM variety if and only $X$ is integral of dimension $n$.

TheOREM 2.9. The constructions of Lemmas 2.7 and 2.8 are mutually inverse and define an equivalence of groupoids between

- the groupoid of all normal polarized GM varieties $(X, H)$ of dimension $n$ and

- the groupoid of all $G M$ data sets $\left(W, V_{6}, V_{5}, L, \mu, \mathbf{q}, \varepsilon\right)$ of dimension $n$ such that the GM intersection $X\left(W, V_{6}, V_{5}, L, \mu, \mathbf{q}, \varepsilon\right)$ is n-dimensional, integral, and normal.

This equivalence induces an injection from the set of isomorphism classes of normal polarized GM varieties into the set of isomorphism classes of GM data sets and also works in families.

Proof. Let $(X, H)$ be a $\mathrm{GM}$ variety, and let $\left(W, V_{6}, V_{5}, L, \mu, \mathbf{q}, \varepsilon\right)$ be the associated GM data. We saw during the proof of Theorem 2.3 that one can write $X$ as in (2.6). Comparing with (2.9), we obtain $X\left(W, V_{6}, V_{5}, L, \mu, \mathbf{q}, \varepsilon\right) \simeq X$. Conversely, start with a GM data set $\left(W, V_{6}, V_{5}, L, \mu, \mathbf{q}, \varepsilon\right)$, and set $X:=X\left(W, V_{6}, V_{5}, L, \mu, \mathbf{q}, \varepsilon\right)$. Applying to $X$ the construction of Theorem 2.3, one gets the original GM data set back. The functoriality and the fact that the equivalence works in families are clear from the construction. 


\section{O. Debarre and A. Kuznetsov}

Remark 2.10. The proof of Lemma 2.8 shows that if $X$ is a normal polarized GM variety, one can always assume $K=\operatorname{Ker}(\mu)$. Thus, a normal GM variety with GM data $\left(W, V_{6}, V_{5}, L, \mu, \mathbf{q}, \varepsilon\right)$ can be written as

for any $v \in V_{6} \backslash V_{5}$.

$$
X=\mathrm{C}_{\mathrm{Ker}(\mu)}\left(\operatorname{Gr}\left(2, V_{5}\right) \cap \mathbf{P}(\mu(W))\right) \cap Q(v)
$$

Recall that $\operatorname{Aut}\left(W, V_{6}, V_{5}, L, \mu, \mathbf{q}, \varepsilon\right)$ is a subgroup of $\mathrm{GL}(W) \times \mathrm{GL}\left(V_{6}\right) \times \mathbb{G}_{\mathrm{m}}$.

Corollary 2.11. Let $(X, H)$ be a normal polarized $G M$ variety, with associated GM data $\left(W, V_{6}, V_{5}, L, \mu, \mathbf{q}, \varepsilon\right)$. There exist an isomorphism

$$
\operatorname{Aut}(X, H) \simeq \operatorname{Aut}\left(W, V_{6}, V_{5}, L, \mu, \mathbf{q}, \varepsilon\right)
$$

of algebraic groups and an exact sequence

$$
1 \rightarrow \mathbb{G}_{\mathrm{m}} \rightarrow \operatorname{Aut}\left(W, V_{6}, V_{5}, L, \mu, \mathbf{q}, \varepsilon\right) \rightarrow \operatorname{Aut}_{H}(X) \rightarrow 1 .
$$

Proof. Follows from Theorem 2.9 and (2.3).

\subsection{Mildly singular GM varieties}

In this section, we give a simpler intrinsic characterization of mildly singular GM varieties which will allow us to extend the Gushel-Mukai classification.

We say that a smooth projective curve $X$ of genus 6 is Clifford general if $X_{\mathbf{C}}$ is not hyperelliptic, nor trigonal, nor a plane quintic.

Proposition 2.12. A smooth projective curve is a GM curve if and only if it is a Clifford general curve of genus 6. Equivalently, its canonical model is an intersection of six quadrics in $\mathbf{P}^{5}$.

Proof. This follows from Theorem 2.3 combined with the Enriques-Babbage theorem [ACGH85, Section III.3].

An analogous characterization holds for GM surfaces. Following Mukai ([Muk02, Definition 3.8] (with a misprint in the English translation), [JK04, Definition 10.1], [GLT15, Definition 1.3]), we say that a complex polarized $\mathrm{K} 3$ surface $(S, H)$ is Brill-Noether general if

$$
h^{0}(S, D) h^{0}(S, H-D)<h^{0}(S, H)
$$

for all divisors $D$ on $S$ not linearly equivalent to 0 or $H$. When $H^{2}=10$, this is equivalent, by [JK04, Proposition 10.5], to the fact that $|H|$ contains a Clifford general smooth curve (by [GL87], all smooth curves in $|H|$ are then Clifford general).

Proposition 2.13. A smooth projective surface $X$ is a GM surface if and only if $X_{\mathbf{C}}$ is a BrillNoether general polarized K3 surface of degree 10.

Proof. By Corollary 2.4, we may assume $\mathbf{k}=\mathbf{C}$. If $X$ is a smooth GM surface, we have $K_{X}=0$ by Theorem 2.3(a), and the resolution (2.4) implies $H^{1}\left(X, \mathscr{O}_{X}\right)=0$; hence $X$ is a $\mathrm{K} 3$ surface. Moreover, a general hyperplane section of $X$ is a GM curve, hence a Clifford general curve of genus 6; hence $X$ is Brill-Noether general. The other direction is proved in Theorem 2.16 below.

If $X$ is a GM variety, any integral hyperplane section of $X$ is also a GM variety. We are going to show that the converse is also true under some additional assumptions. We start with the following result. 


\section{Gushel-Mukai VARIETIES}

LEMma 2.14. If $(X, H)$ is a normal polarized GM variety, it is projectively normal; that is, the canonical maps

$$
\mathrm{S}^{m} H^{0}\left(X, \mathscr{O}_{X}(H)\right) \rightarrow H^{0}\left(X, \mathscr{O}_{X}(m H)\right)
$$

are surjective for all $m \geqslant 0$.

Proof. Take any integer $m$, and consider the twist

$$
\begin{aligned}
0 & \rightarrow \mathscr{O}_{\mathbf{P}(W)}(m-7) \rightarrow \mathscr{O}_{\mathbf{P}(W)}(m-5)^{\oplus 6} \rightarrow \mathscr{O}_{\mathbf{P}(W)}(m-4)^{\oplus 5} \oplus \mathscr{O}_{\mathbf{P}(W)}(m-3)^{\oplus 5} \\
& \rightarrow \mathscr{O}_{\mathbf{P}(W)}(m-2)^{\oplus 6} \rightarrow \mathscr{O}_{\mathbf{P}(W)}(m) \rightarrow \mathscr{O}_{X}(m H) \rightarrow 0
\end{aligned}
$$

by $\mathscr{O}(m)$ of the resolution (2.4). We want to show that the map on global sections induced by the rightmost map is surjective. This holds because, $\mathbf{P}(W)$ being a projective space of dimension $n+4 \geqslant 5$, the cohomology spaces $H^{1}\left(\mathbf{P}(W), \mathscr{O}_{\mathbf{P}(W)}(m-2)\right), H^{2}\left(\mathbf{P}(W), \mathscr{O}_{\mathbf{P}(W)}(m-3)\right)$, $H^{2}\left(\mathbf{P}(W), \mathscr{O}_{\mathbf{P}(W)}(m-4)\right), H^{3}\left(\mathbf{P}(W), \mathscr{O}_{\mathbf{P}(W)}(m-5)\right)$, and $H^{4}\left(\mathbf{P}(W), \mathscr{O}_{\mathbf{P}(W)}(m-7)\right)$ all vanish.

Proposition 2.15. Let $(X, H)$ be a normal polarized variety of dimension $n \geqslant 2$ such that $K_{X}=-(n-2) H$ and $H^{1}\left(X, \mathscr{O}_{X}\right)=0$. If there is a hypersurface $X^{\prime} \subset X$ in the linear system $|H|$ such that $\left(X^{\prime},\left.H\right|_{X^{\prime}}\right)$ is a normal polarized $G M$ variety, $(X, H)$ is also a polarized $G M$ variety.

Proof. Let us verify the conditions of Theorem 2.3. Condition (a) is true by our assumptions, since $H^{n}=H \cdot H^{n-1}=\left(\left.H\right|_{X^{\prime}}\right)^{n-1}=10$. Furthermore, $X^{\prime}$ is projectively normal by Lemma 2.14. Using [Isk77, Lemma 2.9], we conclude that $X$ is also projectively normal. Since $H$ is ample, it is very ample by $[$ Mum70, $\S 1$, p. 38]. The exact sequence

$$
0 \rightarrow \mathscr{O}_{X} \rightarrow \mathscr{O}_{X}(H) \rightarrow \mathscr{O}_{X^{\prime}}(H) \rightarrow 0
$$

and the assumption $H^{1}\left(X, \mathscr{O}_{X}\right)=0$ imply

$$
h^{0}\left(X, \mathscr{O}_{X}(H)\right)=h^{0}\left(X^{\prime}, \mathscr{O}_{X^{\prime}}(H)\right)+1=n+5 .
$$

This proves condition (b), and [Isk77, Lemma 2.10] proves condition (c). Finally, since $\mathscr{U}_{X^{\prime}}$ is simple (because $X^{\prime}$ is a GM variety), the argument from the proof of Theorem 2.3(d) proves that $\mathscr{U}_{X}$ is also simple.

THEOREM 2.16. Let $X$ be a normal, locally factorial, complex projective variety of dimension $n \geqslant 1$, with terminal singularities and $\operatorname{codim}(\operatorname{Sing}(X)) \geqslant 4$, together with an ample Cartier divisor $H$ such that $K_{X} \sim_{\operatorname{lin}}-(n-2) H$ and $H^{n}=10$. If we assume that

- when $n \geqslant 3$, we have $\operatorname{Pic}(X)=\mathbf{Z} H$;

- when $n=2$, the surface $X$ is a Brill-Noether general K3 surface;

- when $n=1$, the genus 6 curve $X$ is Clifford general;

then $X$ is a $G M$ variety.

We will prove a partial converse to these statements in Lemma 2.29 below.

To prove the theorem, we verify that a general hyperplane section of $X$ satisfies the same conditions, then use induction on $n$.

Lemma 2.17. Let $X$ be a normal complex projective variety of dimension $n \geqslant 3$ with terminal singularities, $\operatorname{codim}(\operatorname{Sing}(X)) \geqslant 4$, and with an ample Cartier divisor $H$ such that $H^{n}=10$ and $K_{X} \sim_{\text {lin }}-(n-2) H$. 


\section{O. Debarre and A. Kuznetsov}

The linear system $|H|$ is very ample, and a general $X^{\prime} \in|H|$ satisfies the same conditions as $X$ : the variety $X^{\prime}$ is normal with terminal singularities, $\operatorname{codim}\left(\operatorname{Sing}\left(X^{\prime}\right)\right) \geqslant 4$ and, if $H^{\prime}:=\left.H\right|_{X^{\prime}}$, we have $\left(H^{\prime}\right)^{n-1}=10$ and $K_{X^{\prime}} \sim_{\operatorname{lin}}-(n-3) H^{\prime}$.

Proof. By [Ale91, Proposition 1-1], the linear system $|H|$ is non-empty, and by [Mel99, Remark 2.6], a general $X^{\prime} \in|H|$ is normal with terminal singularities. Moreover, $\left(H^{\prime}\right)^{n-1}=10$ and, by the adjunction formula,

$$
K_{X^{\prime}}=\left.\left(K_{X}+H\right)\right|_{X^{\prime}} \sim_{\operatorname{lin}}-\left.(n-3) H\right|_{X^{\prime}} .
$$

Since $X$ has terminal singularities, we have $H^{1}\left(X, \mathscr{O}_{X}\right)=0$ by the Kawamata-Viehweg vanishing theorem; hence the linear series $\left|H^{\prime}\right|$ is just the restriction of $|H|$ to $X^{\prime}$, and the base loci of $|H|$ and $\left|H^{\prime}\right|$ are the same. Taking successive linear sections, we arrive at a linear section $Y$ of dimension 3 with terminal singularities, $K_{Y} \sim_{\text {lin }}-H_{Y}$, and $H_{Y}^{3}=10$.

Assume that some $x \in \operatorname{Sing}(X)$ is in the base locus of $|H|$. That point $x$ is then on $X^{\prime}$, and, since a Cartier divisor is necessarily singular at a singular point of the ambient variety, $x$ is also singular on $X^{\prime}$. Repeating that argument, we see that $x$ is singular on the threefold $Y$ and still a base point of $\left|H_{Y}\right|=|H|_{Y}$. Again, all divisors in $\left|H_{Y}\right|$ are singular at $x$; hence we are in one of the two cases described in [Mel99, Theorem 2.9]. Since $H_{Y}^{3} \neq 10$ in both of these cases, we get a contradiction. Therefore, $\operatorname{Sing}(X) \cap \operatorname{Base}(|H|)=\varnothing$; hence, by Bertini's theorem, $\operatorname{Sing}\left(X^{\prime}\right)$ still has codimension at least 4 in $X^{\prime}$ and eventually, $Y$ is smooth.

If $\operatorname{Pic}(Y)=\mathbf{Z} H_{Y}$, the pair $\left(Y, H_{Y}\right)$ is projectively normal by [IP99, Corollary 4.1.13]. If not, we use the Mori-Mukai classification of smooth Fano threefolds $Y$ with Picard number at least 2 ([MM83], [IP99, Chapter 12]) and see that there is only one family with anticanonical degree 10: $Y$ must be a divisor of bidegree $(3,1)$ in $\mathbf{P}^{3} \times \mathbf{P}^{1}$, and the pair $\left(Y, H_{Y}\right)$ is again projectively normal.

A repeated use of [Isk77, Lemma 2.9] then implies that in all cases, $(X, H)$ is projectively normal; hence $H$ is very ample by [Mum70, $\S 1$, p. 38].

Lemma 2.18. Let $(X, H)$ be a polarized complex variety of dimension $n \geqslant 2$ which satisfies the hypotheses of Theorem 2.16. A general element of $|H|$ then satisfies the same properties.

Proof. First, assume $n>3$. By Lemma 2.17, we only need to prove that a general $X^{\prime} \in|H|$ is locally factorial and that $\operatorname{Pic}\left(X^{\prime}\right)$ is generated by $H^{\prime}:=\left.H\right|_{X^{\prime}}$. Let $\mathrm{Cl}(X)$ be the group of Weil divisors on $X$, modulo linear equivalence. Since $|H|$ is very ample (Lemma 2.17), restriction of divisors induces, by [RS06, Theorem 1], an isomorphism

$$
\mathrm{Cl}(X) \stackrel{\sim}{\longrightarrow} \mathrm{Cl}\left(X^{\prime}\right) .
$$

Since $X$ is normal and locally factorial, the canonical inclusion $\mathbf{Z H}=\operatorname{Pic}(X) \hookrightarrow \operatorname{Cl}(X)$ is an isomorphism. It follows that the canonical inclusion $\operatorname{Pic}\left(X^{\prime}\right) \hookrightarrow \mathrm{Cl}\left(X^{\prime}\right)$ is also an isomorphism; that is, $X^{\prime}$ is locally factorial and $\operatorname{Pic}\left(X^{\prime}\right)$ is generated by $H^{\prime}$.

When $n=2$, the variety $X$ is a smooth Brill-Noether general K3 surface with a polarization $H$ of degree 10; hence a general element of $|H|$ is a smooth Clifford general curve of genus 6 .

When $n=3$, the variety $X$ is a smooth Fano threefold with $\operatorname{Pic}(X) \simeq \mathbf{Z}$. By [IP99, Corollary 4.1.13], it is an intersection of quadrics. Any smooth hyperplane section $S$ of $X$ is a degree 10 smooth K3 surface which is still an intersection of quadrics. A general hyperplane section of $S$ is still an intersection of quadrics, hence is a Clifford general curve. This proves that $S$ is BrillNoether general. 


\section{Gushel-Mukai VARIETIES}

We can now prove Theorem 2.16.

Proof of Theorem 2.16. We use induction on $n$. The case $n=1$ was proved in Proposition 2.12, so we assume $n \geqslant 2$. A general hyperplane section $X^{\prime}$ of $X$ has the same properties as $X$ by Lemma 2.18, hence is a GM variety by the induction hypothesis. On the other hand, we have $H^{1}\left(X, \mathscr{O}_{X}\right)=0$ : this follows from the Kawamata-Viehweg vanishing theorem for $n \geqslant 3$ and from the fact that $X$ is a $\mathrm{K} 3$ surface for $n=2$. By Proposition 2.15, we conclude that $X$ is a GM variety.

Remark 2.19. If we relax the conditions on the Picard group or on the singularities of $X$, the conclusion of Theorem 2.16 may be false. Indeed, a general divisor of bidegree $(3,1)$ in $\mathbf{P}^{3} \times \mathbf{P}^{1}$ is an example of a smooth Fano threefold of coindex 3 and degree 10 which is not an intersection of quadrics. Further counterexamples can be found in [PCS05]: one is the threefold $T_{7}$ in

Theorem 1.6 of that article, which can be defined as the anticanonical image of the blow-up of a quartic double solid in a line [PCS05, Example 1.11]. It has one ordinary double point, hence has terminal Gorenstein singularities, but is not locally factorial because it is the image of a nontrivial small contraction. Its Picard number is 1 , and $\left|-K_{T_{7}}\right|$ is very ample of degree 10 and projective dimension 7 , but $T_{7}$ is not an intersection of quadrics. In particular, the conclusion of Theorem 2.16 is definitely false for it.

\subsection{Grassmannian hulls}

We fix some more terminology and notation. Given a normal polarized GM variety $(X, H)$ of dimension $n$, we consider the GM data set $\left(W, V_{6}, V_{5}, L, \mu, \mathbf{q}, \varepsilon\right)$ constructed in Lemma 2.7 and define the Grassmannian hull of $X$ by

$$
M_{X}:=\bigcap_{v \in V_{5}} Q(v)=\mathrm{C}_{K} \mathrm{Gr}\left(2, V_{5}\right) \cap \mathbf{P}(W),
$$

where the second equality is (2.9). Since $\operatorname{dim}(X)=n$, the intersection $C_{K} \operatorname{Gr}\left(2, V_{5}\right) \cap \mathbf{P}(W)$ is dimensionally transverse and $M_{X}$ is a variety of degree 5 and dimension $n+1$, defined over the field k. Moreover, by Lemma 2.8, we have

$$
X=M_{X} \cap Q(v)
$$

for any $v \in V_{6} \backslash V_{5}$. Since $X$ is irreducible, so is $M_{X}$.

When $\mu$ is not injective, the Grassmannian hull is a cone. It is convenient to consider also the dimensionally transverse intersection

$$
M_{X}^{\prime}:=\operatorname{Gr}\left(2, V_{5}\right) \cap \mathbf{P}(\mu(W)) .
$$

In terms of $M_{X}^{\prime}$, the structure of a GM variety can be described as follows.

Lemma 2.20. Let $X$ be a $G M$ variety with associated $G M$ data $\left(W, V_{6}, V_{5}, L, \mu, \mathbf{q}, \varepsilon\right)$. Denote $K:=\operatorname{Ker}(\mu)$ and $k:=\operatorname{dim}(K)$, choose $v \in V_{6} \backslash V_{5}$, and set $Q:=Q(v)$.

(a) If $k=0$, we have $X=M_{X}^{\prime} \cap Q$.

(b) If $k>0$, let $\tilde{X}$ be the blow-up of $X$ with center $\mathbf{P}(K) \cap Q \subset X$. The map $\mu$ then induces a regular map $\tilde{\mu}: \tilde{X} \rightarrow M_{X}^{\prime}$ which is generically a $(k-1)$-dimensional quadric bundle if $\mathbf{P}(K) \not \subset Q$ or a $\mathbf{P}^{k-1}$-bundle if $\mathbf{P}(K) \subset Q$.

Proof. The first part is evident, since $M_{X}^{\prime}=M_{X}$ when $K=0$. For the second part, note that the blow-up of the cone $M_{X}=\mathrm{C}_{K}\left(M_{X}^{\prime}\right)$ at its vertex $\mathbf{P}(K)$ is a $\mathbf{P}^{k}$-bundle over $M_{X}^{\prime}$. The blow-up 


\section{O. Debarre and A. Kuznetsov}

of $X$ along $X \cap \mathbf{P}(K)=\mathbf{P}(K) \cap Q(v)$ is the same as the proper preimage of $X$. In particular, it comes with a regular map $\tilde{X} \rightarrow M_{X}^{\prime}$.

If $\mathbf{P}(K) \not \subset Q$, the proper preimage coincides with the total preimage. Hence its fibers are intersections $\mathbf{P}^{k} \cap Q$; that is, generically, they are quadrics of dimension $k-1$. If $\mathbf{P}(K) \subset Q$, the general fiber of the total preimage of $X$ is a quadric $Q \cap \mathbf{P}^{k}$ which contains the hyperplane $\mathbf{P}(K)=\mathbf{P}^{k-1}$. Therefore, the general fiber of the proper preimage is the residual hyperplane.

Later on, we will give more details for the case $k=1$ and $\mathbf{P}(K) \not \subset Q$.

In this article, we are mostly interested in the case of smooth GM varieties. However, for many questions, the condition $\operatorname{codim}(\operatorname{Sing}(X)) \geqslant 4$ is almost as good as smoothness. Keeping in mind possible applications of singular GM varieties, we keep the above assumption as long as possible.

Lemma 2.21. Let $\left(W, V_{6}, V_{5}, L, \mu, \mathbf{q}, \varepsilon\right)$ be a $G M$ data set of dimension $n$. If the associated GM intersection (2.8) is a $G M$ variety $X$ of dimension $n$ with $\operatorname{codim}(\operatorname{Sing}(X)) \geqslant 4$, we have

$$
\operatorname{dim}(\mu(W)) \geqslant \min \{7, n+4\} .
$$

Proof. As in the proof of Lemma 2.8, we can write $X$ as in (2.1) with $K=\operatorname{Ker}(\mu)$. Then, $\mathbf{P}(K)$ is contained in the singular locus of $\mathrm{C}_{\mathrm{K}} \mathrm{Gr}\left(2, V_{5}\right) \cap \mathbf{P}(W)$; hence $\mathbf{P}(K) \cap Q(v)$ is contained in the singular locus of $X$. It has dimension at least $(n+5)-r-2=n+3-r$, where $r:=\operatorname{rank}(\mu)$. Thus the condition codim $(\operatorname{Sing}(X)) \geqslant 4$ implies that either $n+3-r<0$ or $n+3-r \leqslant n-4$. The first is equivalent to $r \geqslant n+4$ and the second to $r \geqslant 7$.

Proposition 2.22. Let $X$ be a $G M$ variety of dimension $n$ with $\operatorname{codim}(\operatorname{Sing}(X)) \geqslant 4$. If $n \geqslant 3$ or $\operatorname{Ker}(\mu) \neq 0$, the variety $M_{X}^{\prime}$ is smooth. Otherwise (if $n \leqslant 2$ and $\operatorname{Ker}(\mu)=0$ ), the variety $M_{X}^{\prime}$ is equal to $M_{X}$ and has finitely many rational double points.

Proof. We begin with a useful observation. Let $W_{0} \subset \bigwedge^{2} V_{5}$ be a linear subspace. Assume that $M^{\prime}:=\operatorname{Gr}\left(2, V_{5}\right) \cap \mathbf{P}\left(W_{0}\right)$ is a dimensionally transverse intersection. Viewing elements of the orthogonal complement $W_{0}^{\perp} \subset \wedge^{2} V_{5}^{\vee}$ as skew-symmetric forms on $V_{5}$, one has (see, for example, [PvdV99, Corollary 1.6])

$$
\operatorname{Sing}\left(M^{\prime}\right)=\mathbf{P}\left(W_{0}\right) \cap \bigcup_{\omega \in W_{0}^{\perp} \backslash\{0\}} \operatorname{Gr}(2, \operatorname{Ker}(\omega)) .
$$

If $\operatorname{dim}\left(W_{0}\right)=10$, then $M^{\prime}=\operatorname{Gr}\left(2, V_{5}\right)$ is smooth. If $\operatorname{dim}\left(W_{0}\right)=9$, either a generator $\omega$ of $W_{0}^{\perp}$ has rank 2 and $\operatorname{Sing}\left(M^{\prime}\right)$ is a 2-plane, or else $M^{\prime}$ is smooth. If $\operatorname{dim}\left(W_{0}\right)=8$, either some $\omega \in W_{0}^{\perp}$ has rank 2, in which case $\operatorname{Gr}(2, \operatorname{Ker}(\omega))$ is a 2-plane contained in the hyperplane $\omega^{\perp}$, whose intersection with $\mathbf{P}\left(W_{0}\right)$ therefore contains a line along which $M^{\prime}$ is singular, or else $M^{\prime}$ is smooth. Finally, if $\operatorname{dim}\left(W_{0}\right) \leqslant 7$, we have $\operatorname{dim}\left(M^{\prime}\right) \leqslant 3$. It follows that in all cases, either $M^{\prime}$ is smooth or $\operatorname{codim}\left(\operatorname{Sing}\left(M^{\prime}\right)\right) \leqslant 3$.

Now, let $X$ be a GM variety such that $M_{X}^{\prime}$ is singular. By the above observation, we have $\operatorname{codim}\left(\operatorname{Sing}\left(M_{X}^{\prime}\right)\right) \leqslant 3$. Since $X=\mathrm{C}_{\operatorname{Ker}(\mu)} M_{X}^{\prime} \cap Q(v)$ for any $v \in V_{6} \backslash V_{5}$ (see Remark 2.10) and $\operatorname{codim}(\operatorname{Sing}(X)) \geqslant 4$, the only possibility is that $Q(v)$ does not intersect the cone over $\operatorname{Sing}\left(M_{X}^{\prime}\right)$. As $Q(v)$ is an ample hypersurface, this is only possible if $\operatorname{Ker}(\mu)=0$ and $\operatorname{Sing}\left(M_{X}^{\prime}\right)$ is finite. In this case, $M_{X}^{\prime}=M_{X}$ has dimension 2 or 3 ; hence $n \leqslant 2$, so we are in the second case of the proposition.

It remains to show that in that case, the only singularities of $M_{X}$ are rational double points. If $n=1$, we have $\operatorname{dim}(W)=6$ and $M_{X}=\operatorname{Gr}\left(2, V_{5}\right) \cap \mathbf{P}(W)$ is a normal surface (it is lci 
with finite singular locus). The restriction map $H^{0}\left(\mathbf{P}(W), \mathscr{O}_{\mathbf{P}(W)}(1)\right) \rightarrow H^{0}\left(M_{X}, \mathscr{O}_{M_{X}}(1)\right)$ is bijective, since its composition with the injection $H^{0}\left(M_{X}, \mathscr{O}_{M_{X}}(1)\right) \rightarrow H^{0}\left(X, \mathscr{O}_{X}(1)\right)$ is. It follows that $M_{X}$ is linearly normal. If it is a cone with vertex $w$, it is contained in $\operatorname{Gr}\left(2, V_{5}\right) \cap \mathbf{T}_{\operatorname{Gr}\left(2, V_{5}\right), w}$ (intersection with a tangent space), which is a 4-dimensional cone with vertex $w$ and span $\mathbf{T}_{\mathrm{Gr}\left(2, V_{5}\right), w} \simeq \mathbf{P}^{6}$. Since $M_{X}$ spans the 5-dimensional $\mathbf{P}(W)$, it is a hyperplane section of this cone, but this contradicts the fact that $M_{X}$ is a surface.

So the quintic surface $M_{X}$ is not a cone; hence it is a del Pezzo surface [Dol12, Proposition 8.1.6 and Definition 8.1.5]. This implies that the singularities of $M_{X}$ are rational double points [Dol12, Theorem 8.1.11].

When $n=2$, the singular points of the threefold $M_{X}$ are rational double points, since a general hyperplane section through such a point has this property, by the case $n=1$.

Remark 2.23. Given a general singular linear section $M^{\prime}$ of $\operatorname{Gr}\left(2, V_{5}\right)$ of dimension $n+1 \geqslant 4$, one checks that a general quadratic section of $M^{\prime}$ is singular in codimension 3 and its singularities are canonical but not terminal.

The following elementary result is useful for checking that Grassmannian hulls are smooth, dimensionally transverse intersections.

Proposition 2.24. Let $W_{0} \subset \bigwedge^{2} V_{5}$ be a linear subspace. Set $M:=\operatorname{Gr}\left(2, V_{5}\right) \cap \mathbf{P}\left(W_{0}\right)$ and $M^{\sharp}:=\operatorname{Gr}\left(2, V_{5}^{\vee}\right) \cap \mathbf{P}\left(W_{0}^{\perp}\right)$. Then $M$ is a smooth, dimensionally transverse intersection if and only if $M^{\sharp}$ has the same property. In that case, if moreover both $M$ and $M^{\sharp}$ are non-empty, one has $\operatorname{dim}(M)+\operatorname{dim}\left(M^{\sharp}\right)=2$.

Proof. The scheme $M$ is a smooth, dimensionally transverse intersection at a point $w \in M$ if and only if $\mathbf{T}_{\mathrm{Gr}\left(2, V_{5}\right), w}$ and $\mathbf{P}\left(W_{0}\right)$ span $\mathbf{P}\left(\bigwedge^{2} V_{5}\right)$. This is not the case exactly when there exists a hyperplane $H \subset \mathbf{P}\left(\bigwedge^{2} V_{5}\right)$ containing both $\mathbf{T}_{\mathrm{Gr}\left(2, V_{5}\right), w}$ and $\mathbf{P}\left(W_{0}\right)$. The pair $(w, H)$ then belongs to the incidence variety of $\operatorname{Gr}\left(2, V_{5}\right)$, whose projective dual is $\operatorname{Gr}\left(2, V_{5}^{\vee}\right)$. It follows that the point $H^{\perp} \in \mathbf{P}\left(\bigwedge^{2} V_{5}^{\vee}\right)$ is in $\operatorname{Gr}\left(2, V_{5}^{\vee}\right)$ and that the hyperplane $w^{\perp}$ contains $\mathbf{T}_{\mathrm{Gr}\left(2, V_{5}\right), H^{\perp}}$. Since $w \in \mathbf{P}\left(W_{0}\right)$, we also have $w^{\perp} \supset \mathbf{P}\left(W_{0}^{\perp}\right)$, so that $M^{\sharp}=\operatorname{Gr}\left(2, V_{5}^{\vee}\right) \cap \mathbf{P}\left(W_{0}^{\perp}\right)$ is not a smooth, dimensionally transverse intersection at $H^{\perp}$.

Remark 2.25. Let $X$ be a GM variety of dimension $n \geqslant 3$ with $\operatorname{codim}(\operatorname{Sing}(X)) \geqslant 4$. By Lemma 2.21, we have $\operatorname{dim}(\mu(W)) \geqslant 7$. By Proposition 2.22, the variety $M_{X}^{\prime}$ is smooth of dimension $6-\operatorname{codim}(\mu(W)) \geqslant 3$. Proposition 2.24 therefore says that $\left(M_{X}^{\prime}\right)^{\sharp}$ is empty (that is, the space $\mu(W)^{\perp} \subset \wedge^{2} V_{5}^{\bigvee}$ contains no forms of rank 2).

For smooth GM curves, $\left(M_{X}^{\prime}\right)^{\sharp}$ is never empty. For smooth GM surfaces, $\left(M_{X}^{\prime}\right)^{\sharp}$ may be non-empty. We finish this subsection with a discussion of these two cases.

Let $X$ be a smooth GM curve. As we will explain in more detail in Section 2.5, either $M_{X}^{\prime}$ is a smooth elliptic quintic curve in $\mathbf{P}(\mu(W))=\mathbf{P}^{4}$, there is a double cover $X \rightarrow M_{X}^{\prime}$ (so that $X$ is bielliptic), $M_{X}$ is the cone over $M_{X}^{\prime}$ and $\left(M_{X}^{\prime}\right)^{\sharp}$ is isomorphic to $M_{X}^{\prime}$, or else $M_{X}=M_{X}^{\prime}$ is a quintic del Pezzo surface in $\mathbf{P}(\mu(W))=\mathbf{P}^{5}$.

Mukai remarked [Muk93, Theorem 5.1(2)] that $\left(M_{X}^{\prime}\right)^{\sharp}$ has a simple geometric interpretation as the set of all $g_{4}^{1}$, that is, movable linear systems of degree 4 on $X$. This allows us to recover a classical result without appealing to the description of del Pezzo quintics as blow-ups of the projective plane (compare with [She89, Proposition 4]). 


\section{O. Debarre And A. KuZnetsov}

Proposition 2.26. Let $X$ be a smooth complex GM curve. The Grassmannian hull $M_{X}$ is smooth; that is, $X$ is the intersection of a smooth quintic del Pezzo surface with a quadric, if and only if $X$ has exactly five distinct $g_{4}^{1}$.

Proof. As we just explained, $X$ is the intersection of a smooth quintic del Pezzo surface with a quadric if and only if $M_{X}^{\prime}$ is a smooth (quintic) surface. By Proposition 2.24, this is the case if and only if $\left(M_{X}^{\prime}\right)^{\#}$ is a smooth scheme of dimension 0. On the other hand, the scheme $W_{4}^{1}(X)$ parametrizing the $g_{4}^{1}$ on $X$ either is infinite, in which case $X$ is bielliptic, or has length 5 . The proposition therefore follows from Mukai's remark [Muk93, Theorem 5.1(2)] that there is a bijection between the sets $\left(M_{X}^{\prime}\right)^{\sharp}$ and $W_{4}^{1}(X)$.

Let $(X, H)$ be a smooth GM surface. As in the case of curves, $M_{X}^{\prime}$ may be singular. By Proposition 2.22, this may happen only when $\mu$ is injective. By Proposition 2.24, the variety $M_{X}=M_{X}^{\prime}$ is then a singular threefold and $\left(M_{X}^{\prime}\right)^{\sharp}$ is non-empty. This has strong consequences for $X$.

More precisely, one can show that $\left(M_{X}^{\prime}\right)^{\sharp}$ is non-empty if and only if the Gushel bundle can be written over $\mathbf{C}$ as an extension

$$
0 \rightarrow \mathscr{O}_{X_{\mathbf{C}}}\left(-h_{2}\right) \rightarrow \mathscr{U}_{X_{\mathbf{C}}} \rightarrow \mathscr{O}_{X_{\mathbf{C}}}\left(-h_{1}\right) \rightarrow 0,
$$

where $h_{1}$ and $h_{2}$ are divisors on $X_{\mathbf{C}}$ such that

$$
h_{1}^{2}=0, \quad h_{1} h_{2}=4, \quad h_{2}^{2}=2, \quad h_{1}+h_{2}=H
$$

(compare with [GLT15, Lemma 1.5]). In particular, the Picard number of $X_{\mathbf{C}}$ must be strictly greater than 1.

\subsection{Locally complete intersection GM varieties}

In the rest of the article, we mostly deal with GM varieties which are lci. This condition can be described in terms of GM data sets.

Definition 2.27. A GM data set $\left(W, V_{6}, V_{5}, L, \mu, \mathbf{q}, \varepsilon\right)$ is called $l c i$ if

- either $\operatorname{Ker}(\mu)=0$, in which case the data set is called ordinary;

- or $\operatorname{dim}(\operatorname{Ker}(\mu))=1$ and the point of $\mathbf{P}(W)$ corresponding to the subspace $\operatorname{Ker}(\mu) \subset W$ does not lie on the quadric $Q(v)$ for any $v \in V_{6} \backslash V_{5}$, in which case the data set is called special.

Proposition 2.28. A normal GM variety is lci if and only if its canonically associated GM data set is lci. In particular, a normal lci GM variety can be written in the form (2.1) with $\operatorname{dim}(K)=1$.

Proof. Since $\operatorname{Gr}\left(2, V_{5}\right)$ is not a complete intersection, the non-lci locus of the cone $\mathrm{C}_{\mathrm{K}} \mathrm{Gr}\left(2, V_{5}\right)$ is precisely its vertex $\mathbf{P}(K)$. Since a GM variety $X$ is a dimensionally transverse intersection (see (2.1)), it is lci if and only if it does not contain points of $\mathbf{P}(K)$, that is, if and only if $X \cap \mathbf{P}(K)=\mathbf{P}(\operatorname{Ker}(\mu)) \cap Q(v)$ is empty. The latter condition can be rephrased as $\operatorname{dim}(\operatorname{Ker}(\mu)) \leqslant 1$ and in case of equality, the corresponding point is not in $Q(v)$.

The next lemma will usually be applied to smooth GM varieties, but as usual we prove it under a weaker assumption. In this form, it is a partial converse to Theorem 2.16.

Lemma 2.29. Let $(X, H)$ be an lci polarized complex GM variety of dimension $n \geqslant 3$. If $\operatorname{codim}(\operatorname{Sing}(X)) \geqslant 4$, the variety $X$ is locally factorial and $\operatorname{Pic}(X)=\mathbf{Z} H$. In particular, the polarization $H$ is the unique GM polarization on $X$; hence $\operatorname{Aut}_{H}(X)=\operatorname{Aut}(X)$. 


\section{Gushel-Mukai VARIETIES}

Proof. By [Gro05, XI, corollaire 3.14], the variety $X$ is locally factorial. It remains to show that $\operatorname{Pic}(X)=\mathbf{Z} H$. First, assume $n=3$, so that $X$ is smooth. By Theorem 2.3 , the variety $X$ is a Fano variety of degree 10 and is an intersection of quadrics. As we already mentioned in the proof of Lemma 2.17, the only Fano threefold of degree 10 and Picard number greater than 1 is not an intersection of quadrics. This proves the claim for $n=3$. When $n>3$, we proceed by induction: a general hyperplane section $X^{\prime}$ of $X$ satisfies the same properties and restriction induces an injection $\operatorname{Pic}(X) \hookrightarrow \operatorname{Pic}\left(X^{\prime}\right)$ [Gro05, XII, corollaire 3.6].

For any lci GM variety $X$, the Gushel sheaf $\mathscr{U}_{X}$ is locally free (we will call it the Gushel bundle) and the Gushel map $X \rightarrow \operatorname{Gr}\left(2, V_{5}\right)$ is regular. Moreover, we are in exactly one of the following cases:

- The map $\mu: W \rightarrow \wedge^{2} V_{5}$ is injective, the Gushel map is a closed embedding, we have $M_{X}=M_{X}^{\prime}$, and (2.1) can be rewritten as

$$
X=\operatorname{Gr}\left(2, V_{5}\right) \cap \mathbf{P}(W) \cap Q(v),
$$

so that $X$ is a quadratic section of $M_{X}$. These GM varieties will be called ordinary. For these varieties,

$$
\operatorname{dim}(X)=\operatorname{dim}(W)-5 \leqslant \operatorname{dim}\left(\bigwedge^{2} V_{5}\right)-5=5 .
$$

- The kernel of $\mu$ has dimension 1 , the Gushel map induces a double covering $X \rightarrow M_{X}^{\prime}$, and $M_{X}=\mathrm{C} M_{X}^{\prime}$. These GM varieties will be called special. For these varieties,

$$
\operatorname{dim}(X)=\operatorname{dim}(W)-5 \leqslant \operatorname{dim}\left(\bigwedge^{2} V_{5}\right)+1-5=6 .
$$

A special GM variety comes with a canonical involution: the involution of the double covering $X \rightarrow M_{X}^{\prime}$.

Let us make an observation. Let $\left(W, V_{6}, V_{5}, L, \mu, \mathbf{q}, \varepsilon\right)$ be an lci GM data set, and set

$$
W_{1}:=\operatorname{Ker}(\mu) \subset W, \quad W_{0}:=W / W_{1} .
$$

Furthermore, let $\mu_{0}: W_{0} \hookrightarrow \bigwedge^{2} V_{5}$ be the embedding induced by $\mu$.

Proposition 2.30. For any lci $G M$ data set $\left(W, V_{6}, V_{5}, L, \mu, \mathbf{q}, \varepsilon\right)$, there is a unique splitting of the canonical exact sequence $0 \rightarrow W_{1} \rightarrow W \rightarrow W_{0} \rightarrow 0$, that is, a direct sum decomposition

$$
W=W_{0} \oplus W_{1},
$$

such that the map q decomposes as

$$
\mathbf{q}=\mathbf{q}_{0}+\mathbf{q}_{1}, \quad \text { where } \quad \mathbf{q}_{0}: V_{6} \rightarrow \mathrm{S}^{2} W_{0}^{\vee} \quad \text { and } \quad \mathbf{q}_{1}: V_{6} \rightarrow \mathrm{S}^{2} W_{1}^{\vee} .
$$

Proof. If the data set is ordinary, we have $W_{1}=0$ and $W_{0}=W$, and there is nothing to prove. If $W_{1}$ is non-zero, it is 1-dimensional. Let $w_{1} \in W_{1}$ be any non-zero vector, and choose an arbitrary $v \in V_{6} \backslash V_{5}$. Since the data set is lci, the quadric $Q(v)$ does not pass through the point $w_{1} \in W_{1}$; hence the linear form $\mathbf{q}(v)\left(w_{1},-\right) \in W^{\vee}$ does not vanish on $w_{1}$ and gives a decomposition $W=W_{0} \oplus W_{1}$ such that $\mathbf{q}(v) \in \mathrm{S}^{2} W_{0}^{\vee} \oplus \mathrm{S}^{2} W_{1}^{\vee} \subset \mathrm{S}^{2} W^{\vee}$. By (2.7), we have $\mathbf{q}\left(V_{5}\right) \subset \mathrm{S}^{2} W_{0}^{\vee}$; hence the decomposition of $W$ does not depend on the choice of $v$, and the image of $\mathbf{q}$ is contained in $\mathrm{S}^{2} W_{0}^{\vee} \oplus \mathrm{S}^{2} W_{1}^{\vee} \subset \mathrm{S}^{2} W^{\vee}$. We let $\mathbf{q}_{0}$ and $\mathbf{q}_{1}$ be the summands of $\mathbf{q}$ corresponding to this direct sum decomposition.

Remark 2.31. One can prove the same result as in Proposition 2.30 after replacing the lci condition by the assumption that for $v \notin V_{5}$, the restriction to $W_{1}:=\operatorname{Ker}(\mu) \subset W$ of the quadratic 


\section{O. Debarre and A. Kuznetsov}

form $\mathbf{q}(v)$ is non-degenerate (this assumption does not depend on the choice of $v$ ). The proof is the same: $W_{0}$ is defined as the orthogonal of $W_{1}$ in $W$ with respect to $\mathbf{q}(v)$.

Corollary 2.32. Let $(X, H)$ be a normal, lci, polarized GM variety. The kernel of the canonical morphism $\operatorname{Aut}_{H}(X) \rightarrow \mathrm{PGL}\left(V_{6}\right)$ is trivial if $X$ is ordinary and is generated by the canonical involution of $X$ if $X$ is special.

Proof. By Corollary 2.11, the group $\operatorname{Aut}_{H}(X)$ is a quotient of the group

$$
\operatorname{Aut}\left(W, V_{6}, V_{5}, L, \mu, \mathbf{q}, \varepsilon\right) \subset \mathrm{GL}(W) \times \mathrm{GL}\left(V_{6}\right) \times \mathbb{G}_{\mathrm{m}}
$$

of automorphisms of its associated GM data set. Such an automorphism $g=\left(g_{W}, g_{V}, g_{L}\right)$ maps to the identity in $\operatorname{PGL}\left(V_{6}\right)$ if its component $g_{V}$ is scalar. Its actions on $V_{5}$, on $\bigwedge^{2} V_{5}$, and hence on $W_{0} \subset \bigwedge^{2} V_{5}$, are then also scalar. For ordinary varieties $X \subset \mathbf{P}\left(W_{0}\right)$, the action of such a $g$ on $X$ is therefore trivial.

For special varieties, since the direct sum decomposition $W=W_{0} \oplus W_{1}$ is canonical, it is preserved by $g_{W}$, and since $W_{1}$ is 1-dimensional, $g_{W}$ acts by multiplication on each summand, by scalars $t_{0}$ and $t_{1}$. Then $g$ acts by multiplication by $t_{0}^{-2}$ on $\mathrm{S}^{2} W_{0}^{\vee}$ and by $t_{1}^{-2}$ on $\mathrm{S}^{2} W_{1}$. Since the map q: $V_{6} \rightarrow \mathrm{S}^{2} W_{0}^{\vee} \oplus \mathrm{S}^{2} W_{1}^{\vee}$ commutes with the action of $g$, and the action of $g$ on $V_{6}$ is scalar, we get $t_{0}^{-2}=t_{1}^{-2}$. Thus $t_{1}= \pm t_{0}$; hence $g$ acts on $\mathbf{P}(W)$ either identically or by a reflection with respect to $\mathbf{P}\left(W_{0}\right)$, that is, by the canonical involution of $X$.

For a special GM variety $X$ of dimension $n \geqslant 2$, the branch locus $X^{\prime}$ of the Gushel map $X \rightarrow M_{X}^{\prime}$ is the dimensionally transverse intersection of $M_{X}^{\prime}$ with a quadric, so it is an ordinary GM variety. This leads to an important birational operation on the set of all GM varieties: interchanging ordinary and special varieties. We formulate this operation on the level of GM data.

Lemma 2.33. If $\left(W, V_{6}, V_{5}, L, \mu, \mathbf{q}, \varepsilon\right)$ is a special lci $G M$ data set, $\left(W_{0}, V_{6}, V_{5}, L, \mu_{0}, \mathbf{q}_{0}, \varepsilon\right)$ is an ordinary lci GM data set.

Conversely, let $\left(W_{0}, V_{6}, V_{5}, L, \mu_{0}, \mathbf{q}_{0}, \varepsilon\right)$ be an ordinary lci $G M$ data set. Let $W_{1}$ be a 1 dimensional vector space, choose an isomorphism $V_{6} / V_{5} \simeq \mathrm{S}^{2} W_{1}^{\vee}$, and let $\mathbf{q}_{1}$ be the composition $V_{6} \rightarrow V_{6} / V_{5} \rightarrow \mathrm{S}^{2} W_{1}^{\vee}$. Then $\left(W_{0} \oplus W_{1}, V_{6}, V_{5}, L, \mu_{0}, \mathbf{q}_{0}+\mathbf{q}_{1}, \varepsilon\right)$ is a special lci GM data set.

If the field $\mathbf{k}$ is quadratically closed, that is, if $\mathbf{k}=\mathbf{k}^{1 / 2}$, these operations define a bijection between the sets of isomorphism classes of special and ordinary GM data sets, respectively.

Proof. The first part is obvious. For the second part, note that different choices of an isomorphism $V_{6} / V_{5} \simeq \mathrm{S}^{2} W_{1}^{\vee}$ produce isomorphic GM data sets (via the isomorphism defined by $g_{V}=\mathrm{id}_{V}$, $g_{W}=\mathrm{id}_{W_{0}}+\sqrt{t} \mathrm{id}_{W_{1}}, g_{L}=\mathrm{id}_{L}$ for an appropriate $\left.t \in \mathbf{k}\right)$.

When $\mathbf{k}=\mathbf{k}^{1 / 2}$, this bijection can be interpreted as a bijection between isomorphism classes of special and ordinary lci GM varieties. We will denote by $X_{\text {ord }}$ the ordinary lci GM intersection associated with a given special lci GM variety $X$ and by $X_{\text {spe }}$ the special lci GM intersection associated with a given ordinary lci GM variety $X$. We define the opposite $X_{\mathrm{opp}}$ of an lci GM variety $X$ by

$$
X_{\mathrm{opp}}:= \begin{cases}X_{\mathrm{ord}} & \text { if } X \text { is special } \\ X_{\mathrm{spe}} & \text { if } X \text { is ordinary. }\end{cases}
$$




\section{GuSHEL-MuKaI VARIETIES}

\section{GM varieties, Lagrangian data sets, and EPW sextics}

Eisenbud-Popescu-Walter (EPW) sextics are special hypersurfaces of degree 6 in $\mathbf{P}\left(V_{6}\right)$ which can be constructed from Lagrangian subspaces $A \subset \bigwedge^{3} V_{6}$. The definition and main properties of EPW sextics can be found in Appendix B. A relation between GM varieties and EPW sextics was found in [IM11]. In this section, we develop the approach of Iliev and Manivel and extend their construction to include both ordinary and special varieties.

\subsection{The discriminant locus}

The easiest way to relate an EPW sextic to a GM variety is via the discriminant locus. Let $(X, H)$ be a normal polarized GM variety of dimension $n$ as in Definition 2.1. By Theorem 2.3, the space $V_{6}$ of quadrics in $\mathbf{P}(W)$ containing $X$ is 6 -dimensional. We define $\widetilde{\operatorname{Disc}}(X)$ as the subscheme of $\mathbf{P}\left(V_{6}\right)$ of singular quadrics containing $X$. It is either $\mathbf{P}\left(V_{6}\right)$ or a hypersurface of degree $\operatorname{dim}(W)=n+5$, and in the latter case, the multiplicity in $\widetilde{\operatorname{Disc}}(X)$ of the hyperplane $\mathbf{P}\left(V_{5}\right)$ of (restrictions of) Plücker quadrics is at least the corank of a general such quadric, which is at least $\operatorname{dim}(W)-6=n-1$. We define the discriminant locus $\operatorname{Disc}(X)$ as follows: if $\widetilde{\operatorname{Disc}}(X)=\mathbf{P}\left(V_{6}\right)$, we set $\operatorname{Disc}(X)=\mathbf{P}\left(V_{6}\right)$; otherwise (and we will see in Corollary 3.19 that this is almost always the case), we set

$$
\operatorname{Disc}(X):=\widetilde{\operatorname{Disc}}(X)-(n-1) \mathbf{P}\left(V_{5}\right) .
$$

This is a sextic hypersurface in $\mathbf{P}\left(V_{6}\right)$.

Theorem 3.1 (Iliev-Manivel). Let $X$ be a general (smooth and ordinary) complex GM variety of dimension $n \in\{3,4,5\}$. The discriminant locus $\operatorname{Disc}(X) \subset \mathbf{P}\left(V_{6}\right)$ is an EPW sextic.

The result is proved in [IM11, Proposition 2.1 and Lemma 2.2] for general GM fivefolds by explicitly constructing a Lagrangian $A \subset \wedge^{3} V_{6}$ such that $\operatorname{Disc}(X)=Y_{A}$ (see also [OGr08a, Proposition 2.18]). This construction extends to general GM varieties of dimensions 3 , 4 , and 5 [IM11, Proposition 2.4]. In the next section, we present a version of this construction which works better in families and allows us to treat at the same time both ordinary and special GM varieties. In particular, in Proposition 3.18, we give an extension of Theorem 3.1.

For the time being, we examine the discriminant locus of GM curves and relate the discriminant locus of a GM variety to that of its opposite as defined in (2.14).

Proposition 3.2. Let $X$ be a smooth GM curve. The discriminant locus $\operatorname{Disc}(X)$ is a reduced sextic hypersurface and

- if $X$ is ordinary, $\operatorname{Disc}(X)$ is geometrically integral and normal;

- if $X$ is special (that is, bielliptic), Disc $(X)$ is the union of the Plücker hyperplane $\mathbf{P}\left(V_{5}\right)$ and a geometrically integral quintic hypersurface.

Proof. We may assume $\mathbf{k}=\mathbf{C}$. A local calculation shows that the Zariski tangent space to $\widetilde{\operatorname{Disc}}(X)$ at a point corresponding to a quadric $Q \subset \mathbf{P}(W) \simeq \mathbf{P}^{5}$ containing $X$ has dimension greater than 4 if and only if

(a) either $Q$ has corank 1 but its vertex is on $X$;

(b) or else $Q$ has corank $\geqslant 2$ (hence rank $\leqslant 4$ ).

Case 1: The curve $X$ is ordinary. In case (a), the quadric $Q$ must be a Plücker quadric, because otherwise, $X$ would be equal to $M_{X} \cap Q$ and would be singular at the vertex of $Q$. But 


\section{O. Debarre And A. KuZnetsov}

corank 1 quadrics with vertex at $w \in X$ are in one-to-one correspondence with smooth quadrics containing the projection of $M_{X}$ from $w$. This projection is a del Pezzo surface of degree 4 , hence is the base locus of a pencil of quadrics in $\mathbf{P}^{4}$. Therefore, these quadrics form an open subset in a ruled surface with base $X$.

We now examine case (b). The locus of quadrics of rank at most 4 containing $X$ was studied extensively in [AH81]. It contains five 2-planes (one for each $g_{4}^{1}$ on $X$, counted with multiplicities; [AH81, (5.10)] ) of Plücker quadrics and one more irreducible surface which comes from the singular points of the theta divisor of the Jacobian of $X$, that is, from the $g_{5}^{1}$ on $X$.

All in all, this proves that the singular locus of $\operatorname{Disc}(X)$ has dimension 2 . It follows that $\operatorname{Disc}(X)$ is an integral normal sextic hypersurface.

Case 2: The curve $X$ is bielliptic. In case (a), the quadric $Q$ must again be a Plücker quadric. It is then also singular at the vertex of $M_{X}$, hence cannot be of corank 1 . So case (a) is impossible.

Plücker quadrics are all singular at the vertex and have general corank 1 ; hence $\mathbf{P}\left(V_{5}\right)$ is a reduced component of $\operatorname{Disc}(X)$. Write $\operatorname{Disc}(X)=\mathbf{P}\left(V_{5}\right)+D$, where $D$ is a quintic hypersurface not containing $\mathbf{P}\left(V_{5}\right)$. Then $\operatorname{Sing}(\operatorname{Disc}(X)) \cap \mathbf{P}\left(V_{5}\right)=\mathbf{P}\left(V_{5}\right) \cap D$ corresponds to singular quadrics containing the quintic elliptic curve $M_{X}^{\prime}$, and this was proved [AH81, discussion above (5.6)] to be an integral quintic hypersurface. It follows that $D$ is also an integral quintic hypersurface.

Lemma 3.3. Let $X$ be a special lci GM variety with associated ordinary variety $X_{\text {ord }}$. Then $\operatorname{Disc}(X)=\operatorname{Disc}\left(X_{\text {ord }}\right)$.

Proof. Let $\left(V_{6}, V_{5}, L, W, \mu, \mathbf{q}, \varepsilon\right)$ be the GM data associated with $X$. Let $W=W_{0} \oplus W_{1}$ be the canonical direct sum decomposition of Proposition 2.30, and let $\mathbf{q}=\mathbf{q}_{0}+\mathbf{q}_{1}$ be the decomposition of q. By Lemma 2.33, the ordinary GM variety $X_{\text {ord }}$ is determined by $\mathbf{q}_{0}: V_{6} \rightarrow \mathrm{S}^{2} W_{0}^{\vee}$. In particular, the rank of $\mathbf{q}(v)$ is the sum of the ranks of $\mathbf{q}_{0}(v)$ and $\mathbf{q}_{1}(v)$. By Lemma 2.33, the map $\mathbf{q}_{1}$ vanishes on $V_{5}$ and induces an isomorphism $V_{6} / V_{5} \simeq \mathrm{S}^{2} W_{1}^{\vee}$. Therefore, the rank of $\mathbf{q}(v)$ equals the rank of $\mathbf{q}_{0}(v)$ for Plücker quadrics, and increases by 1 for non-Plücker quadrics, which means that the corank of Plücker quadrics increases by 1 , and the corank of non-Plücker quadrics stays the same. This shows the lemma.

\subsection{GM data and Lagrangian data}

In this section, we construct, following [IM11], a bijection between the set of lci GM data and the set of what we call (extended) Lagrangian data.

Consider a 6 -dimensional vector space $V_{6}$, and endow the space $\wedge^{3} V_{6}$ with the canonical $\operatorname{det}\left(V_{6}\right)$-valued symplectic form given by the wedge product.

Definition 3.4. A Lagrangian data set is a collection $\left(V_{6}, V_{5}, A\right)$, where

- $V_{6}$ is a $\mathbf{k}$-vector space of dimension 6 ,

- $V_{5} \subset V_{6}$ is a hyperplane,

- $A \subset \bigwedge^{3} V_{6}$ is a Lagrangian subspace.

An isomorphism of Lagrangian data sets between $\left(V_{6}, V_{5}, A\right)$ and $\left(V_{6}^{\prime}, V_{5}^{\prime}, A^{\prime}\right)$ is a linear isomorphism $\varphi: V_{6} \stackrel{\sim}{\longrightarrow} V_{6}^{\prime}$ such that $\varphi\left(V_{5}\right)=V_{5}^{\prime}$ and $\left(\bigwedge^{3} \varphi\right)(A)=A^{\prime}$.

A natural extension of the Iliev-Manivel construction gives a bijection between the set of isomorphism classes of Lagrangian data sets and the set of isomorphism classes of ordinary 


\section{Gushel-Mukai VARIETIES}

GM data sets. Using the bijection of Lemma 2.33, one can also use Lagrangian data sets to parameterize all special GM data sets. However, to deal simultaneously with ordinary and special GM varieties, one needs a generalization of the Iliev-Manivel construction. We suggest such a generalization; it uses an additional Lagrangian subspace which encodes the type of a GM variety.

To lighten the notation (and for forward compatibility), we set

$$
L:=\left(V_{6} / V_{5}\right)^{\vee}
$$

and endow the space $\mathbf{k} \oplus L$ with the canonical $L$-valued symplectic form. Note that the group $\operatorname{GL}(L) \simeq \mathbb{G}_{\mathrm{m}}$ acts naturally on the Lagrangian Grassmannian $\operatorname{LGr}(\mathbf{k} \oplus L) \simeq \mathbf{P}^{1}$.

Definition 3.5. An extended Lagrangian data set is a collection $\left(V_{6}, V_{5}, A, A_{1}\right)$, where $\left(V_{6}, V_{5}, A\right)$ is a Lagrangian data set and $A_{1} \subset \mathbf{k} \oplus L$ is a Lagrangian subspace.

An isomorphism of extended Lagrangian data sets between $\left(V_{6}, V_{5}, A, A_{1}\right)$ and $\left(V_{6}^{\prime}, V_{5}^{\prime}, A^{\prime}, A_{1}^{\prime}\right)$ consists of an isomorphism between $\left(V_{6}, V_{5}, A\right)$ and $\left(V_{6}^{\prime}, V_{5}^{\prime}, A^{\prime}\right)$ and an element $t \in \mathbb{G}_{\mathrm{m}}$ such that $t\left(A_{1}\right)=A_{1}^{\prime}$.

The $\operatorname{GL}(L)$-action on the line $\operatorname{LGr}(\mathbf{k} \oplus L) \simeq \mathbf{P}^{1}$ has three orbits. To simplify the notation, we choose a coordinate on this line such that

- the subspace $\mathbf{k} \subset \mathbf{k} \oplus L$ corresponds to the point $\{\infty\} \in \mathbf{P}^{1}$;

- the subspace $L \subset \mathbf{k} \oplus L$ corresponds to the point $\{0\} \in \mathbf{P}^{1}$.

The points $\{0\}$ and $\{\infty\}$ are two of the $\operatorname{GL}(L)$-orbits, and the point $\{1\} \in \mathbf{P}^{1}$ is in the third orbit. To simplify the notation, we will sometimes write $A_{1}=\infty, A_{1}=0$, and $A_{1}=1$ instead of $A_{1}=\mathbf{k}, A_{1}=L$, and $A_{1} \neq \mathbf{k}, L$, respectively.

Theorem 3.6. For any field $\mathbf{k}$, there is a functor (defined in the proof) from the groupoid of lci GM data sets to the groupoid of Lagrangian data sets. It induces a bijection between the set of isomorphism classes of ordinary GM data sets and the set of isomorphism classes of Lagrangian data sets.

If $\mathbf{k}$ is quadratically closed, the functor extends to a bijection between the set of isomorphism classes of lci GM data sets and the set of isomorphism classes of extended Lagrangian data sets with $A_{1} \neq \infty$.

Remark 3.7. The construction of GM data sets can also be applied to Lagrangian data sets with $A_{1}=\infty$; however, the GM data set obtained from this is not lci, and the lci condition is crucial for the inverse construction, since we need the canonical decomposition $W=W_{0} \oplus W_{1}$ of Proposition 2.30.

Before describing the construction, we need one more piece of notation. The canonical projection

$$
\lambda: V_{6} \rightarrow V_{6} / V_{5}=L^{\vee}
$$

defines, by the Leibniz rule, for all $p \geqslant 1$, maps $\lambda_{p}: \bigwedge^{p} V_{6} \rightarrow \bigwedge^{p-1} V_{5} \otimes L^{\vee}$. They fit into exact sequences

$$
0 \rightarrow \bigwedge^{p} V_{5} \hookrightarrow \bigwedge^{p} V_{6} \stackrel{\lambda_{p}}{\longrightarrow} \bigwedge^{p-1} V_{5} \otimes L^{\vee} \rightarrow 0 .
$$

Proof of Theorem 3.6. We start by explaining how to construct a GM data set from an extended Lagrangian data set. After that, we explain the inverse construction and discuss its functoriality. 


\section{O. Debarre And A. KuZnetsov}

Let $\left(V_{6}, V_{5}, A, A_{1}\right)$ be an extended Lagrangian data set. We use the notation (3.2), and we choose an arbitrary isomorphism

$$
\varepsilon: \operatorname{det}\left(V_{5}\right) \stackrel{\sim}{\longrightarrow} L^{\otimes 2}
$$

Consider the maps

$$
\begin{aligned}
& A \hookrightarrow \bigwedge^{3} V_{6} \stackrel{\lambda_{3}}{\longrightarrow} \bigwedge^{2} V_{5} \otimes L^{\vee}, \\
& A_{1} \longleftrightarrow \mathbf{k} \oplus L \stackrel{\mathrm{pr}_{1}}{\longrightarrow} \mathbf{k} .
\end{aligned}
$$

Let $W_{0}$ and $W_{1}$ be their respective images, so that we have canonical factorizations

$$
\begin{aligned}
& A \longrightarrow W_{0} \stackrel{\mu_{0}}{\longleftrightarrow} \bigwedge^{2} V_{5} \otimes L^{\vee}, \\
& A_{1} \longrightarrow W_{1} \stackrel{\mu_{1}}{\longleftrightarrow} \mathbf{k}
\end{aligned}
$$

(defining the maps $\mu_{0}$ and $\mu_{1}$ ). We set

$$
W=W_{0} \oplus W_{1}, \quad \mu=\mu_{0} \oplus \mu_{1} .
$$

We have, by definition,

$$
\operatorname{Ker}\left(A \rightarrow W_{0}\right)=A \cap \bigwedge^{3} V_{5}, \quad \operatorname{Ker}\left(A_{1} \rightarrow W_{1}\right)=A_{1} \cap L .
$$

It remains to define the map q: $V_{6} \rightarrow \mathrm{S}^{2} W^{\vee}$. For this, we first define

$$
\begin{aligned}
& \tilde{\mathbf{q}}_{0}: V_{6} \otimes \mathrm{S}^{2} A \rightarrow \mathbf{k}, \quad \tilde{\mathbf{q}}_{0}(v)\left(\xi_{1}, \xi_{2}\right):=-\varepsilon\left(\lambda_{4}\left(v \wedge \xi_{1}\right) \wedge \lambda_{3}\left(\xi_{2}\right)\right), \\
& \tilde{\mathbf{q}}_{1}: V_{6} \otimes \mathbf{S}^{2} A_{1} \rightarrow \mathbf{k}, \quad \tilde{\mathbf{q}}_{1}(v)\left(x_{1}, x_{1}^{\prime}, x_{2}, x_{2}^{\prime}\right):=\lambda(v) x_{1} x_{2}^{\prime} .
\end{aligned}
$$

It is not immediately clear that $\tilde{\mathbf{q}}_{0}(v)$ and $\tilde{\mathbf{q}}_{1}(v)$ are symmetric in their arguments; we will show later that this follows from the Lagrangian property of $A$ and $A_{1}$, so the above definition makes sense.

For each $v \in V_{6}$ and $i \in\{0,1\}$, the kernel of the quadratic form $\tilde{\mathbf{q}}_{i}(v)$ contains the kernel of the projections $A \rightarrow W_{0}$ and $A_{1} \rightarrow W_{1}$. Indeed, if $\xi_{2} \in A \cap \bigwedge^{3} V_{5}$, we have $\lambda_{3}\left(\xi_{2}\right)=0$; hence $\tilde{\mathbf{q}}_{0}(v)\left(\xi_{1}, \xi_{2}\right)=0$ for any $\xi_{1} \in A$. Analogously, if $\left(x_{1}, x_{1}^{\prime}\right) \in L$, that is, $x_{1}=0$, then $\tilde{\mathbf{q}}_{1}(v)\left(x_{1}, x_{1}^{\prime}, x_{2}, x_{2}^{\prime}\right)=0$ for any $\left(x_{2}, x_{2}^{\prime}\right) \in A_{1}$. This means that $\tilde{\mathbf{q}}_{i}(v)$, considered as a map $\mathrm{S}^{2} A \rightarrow \mathbf{k}$ or $\mathrm{S}^{2} A_{1} \rightarrow \mathbf{k}$, factors through $\mathrm{S}^{2} W_{i}$ and thus defines a quadratic form $\mathbf{q}_{i}(v) \in \mathrm{S}^{2} W_{i}^{\vee}$. We finally define

$$
\mathbf{q}=\mathbf{q}_{0}+\mathbf{q}_{1}: V_{6} \rightarrow \mathrm{S}^{2} W_{0}^{\vee} \oplus \mathrm{S}^{2} W_{1}^{\vee} \subset \mathrm{S}^{2} W^{\vee} .
$$

Let us show that $\left(W, V_{6}, V_{5}, L, \mu, \mathbf{q}, \varepsilon\right)$ is a GM data set. It only remains to check that $\mathbf{q}$ is symmetric (a fact which we already used a couple of times) and that the relation (2.7) holds.

For the symmetry of $\mathbf{q}$, we use the Lagrangian-quadratic correspondence (see Appendix C). We set $\mathbb{V}=\mathbb{V}_{0} \oplus \mathbb{V}_{1}:=\bigwedge^{3} V_{6} \oplus(\mathbf{k} \oplus L)$, a $\mathbf{Z} / 2$-graded vector space endowed with the $L$-valued symplectic form

$$
\omega\left(\left(\xi, x, x^{\prime}\right),\left(\eta, y, y^{\prime}\right)\right)=\varepsilon\left(\lambda_{6}(\xi \wedge \eta)\right)+y x^{\prime}-x y^{\prime} .
$$

Take $v \notin V_{5}$, so that $\lambda(v) \neq 0$, and consider the $\mathbf{Z} / 2$-graded Lagrangian direct sum decomposition

$$
\mathbb{V}=\left(\bigwedge^{3} V_{5} \oplus L\right) \oplus\left(\left(v \wedge \bigwedge^{2} V_{5}\right) \oplus \mathbf{k}\right)
$$

Since $\hat{A}:=A \oplus A_{1} \subset \mathbb{V}$ is another $\mathbf{Z} / 2$-graded Lagrangian subspace, the corresponding quadratic form (as defined in Proposition C.1) evaluated on elements $\left(\xi_{1}, x_{1}, x_{1}^{\prime}\right),\left(\xi_{2}, x_{2}, x_{2}^{\prime}\right) \in \hat{A}$ is $\omega\left(\operatorname{pr}_{1}\left(\xi_{1}, x_{1}, x_{1}^{\prime}\right), \operatorname{pr}_{2}\left(\xi_{2}, x_{2}, x_{2}^{\prime}\right)\right.$ ) (its symmetry follows from the Lagrangian property of $\hat{A}$; see 


\section{Gushel-Mukai VARIETIES}

the proof of Proposition C.1). For the decomposition (3.4), the projections are given by

$$
\begin{aligned}
& \operatorname{pr}_{1}\left(\xi_{1}, x_{1}, x_{1}^{\prime}\right)=\left(\lambda(v)^{-1} \lambda_{4}\left(v \wedge \xi_{1}\right), x_{1}^{\prime}\right) \in\left(\bigwedge^{3} V_{5}\right) \oplus L, \\
& \operatorname{pr}_{2}\left(\xi_{2}, x_{2}, x_{2}^{\prime}\right)=\left(\lambda(v)^{-1} v \wedge \lambda_{3}\left(\xi_{2}\right), x_{2}\right) \in\left(v \wedge \wedge^{2} V_{5}\right) \oplus \mathbf{k} .
\end{aligned}
$$

Substituting these into (3.3), we obtain the form $\tilde{\mathbf{q}}_{0}+\tilde{\mathbf{q}}_{1}$ up to a rescaling by $\lambda(v)$. It follows that for $v \notin V_{5}$, the forms $\tilde{\mathbf{q}}_{i}(v)$ and $\mathbf{q}_{i}(v)$ are symmetric. By continuity, the same is true for $v \in V_{5}$. On the other hand, for $v \in V_{5}$ (so that $\lambda(v)=0$ ), we have $\tilde{\mathbf{q}}_{1}(v)=0$ and

$$
\tilde{\mathbf{q}}_{0}(v)\left(\xi_{1}, \xi_{2}\right)=-\varepsilon\left(\lambda_{4}\left(v \wedge \xi_{1}\right) \wedge \lambda_{3}\left(\xi_{2}\right)\right)=\varepsilon\left(v \wedge \lambda_{3}\left(\xi_{1}\right) \wedge \lambda_{3}\left(\xi_{2}\right)\right)
$$

By the definition of $\mu$, this implies

$$
\mathbf{q}(v)\left(w_{1}, w_{2}\right)=\mathbf{q}_{0}(v)\left(w_{1}, w_{2}\right)=\varepsilon\left(v \wedge \mu_{0}\left(w_{1}\right) \wedge \mu_{0}\left(w_{2}\right)\right),
$$

which proves (2.7) (and gives another proof of the symmetry of $\mathbf{q}(v)$ for $v \in V_{5}$ ).

Let us also show that this GM data set is lci. We have $W_{1}=0$ if $A_{1}=L$ and $W_{1}=A_{1}$ otherwise. But the quadratic form $\tilde{\mathbf{q}}_{1}$ on $A_{1}$ is non-trivial unless $A_{1}=L$ or $A_{1}=\mathbf{k}$. So the only case where $W_{1} \neq 0$ and $\mathbf{q}_{1}=0$ simultaneously (the non-lci case) is the case $A_{1}=\mathbf{k}$, which is excluded from our consideration.

Finally, if we rescale $\varepsilon$ by $t \in \mathbf{k}^{\times}$, the map $\mathbf{q}_{0}$ will also be rescaled by $t$, while the other data will not change. But the action of the element $\left(\varphi_{V}, \varphi_{W}, \varphi_{L}\right) \in \mathrm{GL}\left(V_{6}\right) \times \mathrm{GL}(W) \times \operatorname{GL}(L)$ defined by $\varphi_{V}=t \mathrm{id}_{V}, \varphi_{W}=\mathrm{id}_{W_{0}}+\sqrt{t} \mathrm{id}_{W_{1}}, \varphi_{L}=t^{2} \mathrm{id}_{L}$ precisely realizes such a rescaling (this is the only place where we use the assumption $\mathbf{k}=\mathbf{k}^{1 / 2}$; note, however, that it is unnecessary if $W_{1}=0$, that is, for ordinary GM data sets). This means that different choices of $\varepsilon$ produce isomorphic GM data sets.

We now explain the inverse construction. Let $\left(W, V_{6}, V_{5}, L, \mu, \mathbf{q}, \varepsilon\right)$ be a GM data set. Let $W=W_{0} \oplus W_{1}$ and $\mathbf{q}=\mathbf{q}_{0}+\mathbf{q}_{1}$ be the canonical direct sum decompositions of Proposition 2.30. Choose an arbitrary embedding

$$
\mu_{1}: W_{1} \hookrightarrow \mathbf{k}
$$

consider the maps

$$
\begin{gathered}
V_{5} \otimes W \otimes L \stackrel{f_{1}}{\longrightarrow} \bigwedge^{3} V_{5} \oplus L \oplus \underset{\downarrow}{\downarrow f_{3}}\left(V_{6} \otimes W \otimes L\right) \stackrel{f_{2}}{\longrightarrow} W^{\vee} \otimes L \\
\bigwedge^{3} V_{6} \oplus(\mathbf{k} \oplus L)=\mathbb{V}
\end{gathered}
$$

defined by

$$
\begin{aligned}
f_{1}(v \otimes w \otimes l) & =\left(-v \wedge \mu_{0}(l \otimes w), 0, v \otimes w \otimes l\right), \\
f_{2}\left(\xi, x^{\prime}, v \otimes w \otimes l\right)\left(w^{\prime}\right) & =\varepsilon\left(\xi \wedge \mu_{0}\left(w^{\prime}\right)\right)+\mu_{1}\left(w^{\prime}\right) \otimes x^{\prime}+\mathbf{q}(v)\left(w, w^{\prime}\right) \otimes l, \\
f_{3}\left(\xi, x^{\prime}, v \otimes w \otimes l\right) & =\left(\xi+v \wedge \mu_{0}(l \otimes w), l \otimes \lambda(v) \mu_{1}(w), x^{\prime}\right),
\end{aligned}
$$

(note that $\lambda(v) \in V_{6} / V_{5}=L^{\vee}$, so $l \otimes \lambda(v) \in \mathbf{k}$ ), and define

$$
\hat{A}:=f_{3}\left(\operatorname{Ker}\left(f_{2}\right)\right) \subset \mathbb{V} .
$$

We will check below that $\hat{A} \subset \mathbb{V}$ is a graded Lagrangian subspace.

We introduce gradings on the terms of (3.5) as follows. On the leftmost and the rightmost terms, the grading is induced by the direct sum decomposition $W=W_{0} \oplus W_{1}$; in the middle column, $\wedge^{3} V_{5} \oplus\left(V_{6} \otimes W_{0} \otimes L\right)$ and $\bigwedge^{3} V_{6}$ are the even parts, while $L \oplus\left(V_{6} \otimes W_{1} \otimes L\right)$ and $\mathbf{k} \oplus L$ 


\section{O. Debarre and A. Kuznetsov}

are the odd parts. The gradings are preserved by all the maps. In particular, $\hat{A}$ is a direct sum $\hat{A}=A \oplus A_{1}$ with $A \subset \mathbb{V}_{0}=\bigwedge^{3} V_{6}$ and $A_{1} \subset \mathbb{V}_{1}=\mathbf{k} \oplus L$.

Note that $f_{2} \circ f_{1}=0$ and $f_{3} \circ f_{1}=0$. The second equality is obvious, and the first follows from $\mathbf{q}(v)\left(w, w^{\prime}\right)=\varepsilon\left(v \wedge \mu(w) \wedge \mu\left(w^{\prime}\right)\right)$ for all $v \in V_{5}$ and $w, w^{\prime} \in W$, a reformulation of (2.7). These equalities imply

$$
\hat{A}=f_{3}\left(\operatorname{Ker}\left(\operatorname{Coker}\left(f_{1}\right) \stackrel{f_{2}}{\longrightarrow} W^{\vee}\right)\right) .
$$

Since the third component of $f_{1}$ is the natural embedding $V_{5} \otimes W \otimes L \rightarrow V_{6} \otimes W \otimes L$, choosing a vector $v_{0} \in V_{6} \backslash V_{5}$ splits this map, and we can rewrite $\hat{A}$ as the vector space fitting into the exact sequence

$$
0 \rightarrow \hat{A} \rightarrow\left(\bigwedge^{3} V_{5} \oplus L\right) \oplus\left(v_{0} \otimes W \otimes L\right) \rightarrow W^{\vee} \otimes L \rightarrow 0
$$

which coincides with the exact sequence (C.3), written for the Lagrangian decomposition (3.4) with $v=v_{0}$. Moreover, the embedding of $\hat{A}$ into $\mathbb{V}$ induced by $f_{3}$ coincides with the embedding discussed in the line after (C.3). So Lemma C.2 proves that $\hat{A}$ is Lagrangian.

Finally, we check how the constructed extended Lagrangian data set depends on the choice of $\mu_{1}$. The even part (with respect to the $\mathbf{Z} / 2$-grading) of (3.5) does not depend on this choice, hence the same is true for $A$. This shows that the constructed Lagrangian data set $\left(V_{6}, V_{5}, A\right)$ does not depend on choices and is functorial (an isomorphism of GM data sets induces an isomorphism of the associated Lagrangian data sets).

On the other hand, a simple computation shows that $A_{1}=L$ (that is, $A_{1}$ corresponds to the point $0 \in \mathbf{P}^{1}$ ) if and only if $W_{1}=0$; moreover, $A_{1}=\mathbf{k}$ (that is, $A_{1}$ corresponds to the point $\infty \in \mathbf{P}^{1}$ ) if and only if $W_{1} \neq 0$ and $\mathbf{q}_{1}=0$ (which is the non-lci case); otherwise, $A_{1}$ corresponds to a point of $\mathbf{P}^{1} \backslash\{0, \infty\}$. From this, it is clear that different choices of $\mu_{1}$ produce isomorphic extended Lagrangian data sets.

The two constructions we explained are mutually inverse; this follows from Lemma C.2. This proves the existence of a bijection (if the field $\mathbf{k}$ is quadratically closed) between the sets of isomorphism classes of lci GM data sets and extended Lagrangian data sets .

It remains to show that for any field $\mathbf{k}$, we have a bijection between the sets of isomorphism classes of ordinary GM data sets and Lagrangian data sets. For this, note that the conditions $A_{1}=L$ and $W_{1}=0$ are equivalent and that the map from the set of isomorphism classes of Lagrangian data sets to the set of isomorphism classes of GM data sets is well defined for any field $\mathbf{k}$. The latter follows from the observation that when $W_{1}=0$, a rescaling of $\varepsilon$ by $t \in \mathbf{k}^{\times}$ can be realized by an automorphism $\varphi_{V}=t, \varphi_{W}=1$, and $\varphi_{L}=t^{2}$ of GM data sets; hence the isomorphism class of the obtained GM data set does not depend on the choice of $\varepsilon$.

Remark 3.8. The functor between the groupoid of ordinary GM data sets and the groupoid of ordinary GM data sets defined in Theorem 3.6 is not an equivalence. First, it takes an automorphism $\left(g_{V}, g_{W}, g_{L}\right)=(1,-1,-1)$ to the identity automorphism of the associated Lagrangian data set, hence is not faithful; second, to lift an automorphism $g_{V}$ of the Lagrangian data set associated with a given ordinary $\mathrm{GM}$ data set $\left(W, V_{6}, V_{5}, L, \mu, \mathbf{q}, \varepsilon\right)$ to an automorphism of that GM data set, one has to extract a square root of $\operatorname{det}\left(\left.g_{V}\right|_{V_{5}}\right)$ (to define $\left.g_{L}\right)$.

Remark 3.9. One can generalize Theorem 3.6 by weakening the lci assumption for GM data sets as in Remark 2.31, but one then has to further modify the definition of Lagrangian data set. To be more precise, fix a vector space $K$, consider the symplectic space $\mathbb{V}_{1}(K):=K \oplus\left(K^{\vee} \otimes L\right)$ with the canonical $L$-valued symplectic form (with the isomorphism relation for Lagrangian subspaces $A_{1} \subset \mathbb{V}_{1}(K)$ induced by the natural action of the group $\mathrm{GL}(K)$ on $\mathbb{V}_{1}(K)$ ), and define 


\section{GuSHEL-MuKaI VARIETIES}

$K$-Lagrangian data sets as quadruples $\left(V_{6}, V_{5}, A, A_{1}\right)$, where $A \in \operatorname{LGr}\left(\mathbb{V}_{0}\right)$ and $A_{1} \in \operatorname{LGr}\left(\mathbb{V}_{1}(K)\right)$. Then, there is a bijection between the set of isomorphism classes of $K$-Lagrangian data sets such that $A_{1} \cap K=0$ and the set of isomorphism classes of GM data sets such that $\left.\mathbf{q}(v)\right|_{\operatorname{Ker}(\mu)}$ is non-degenerate for (all) $v \notin V_{5}$ and $\operatorname{dim}(\operatorname{Ker}(\mu)) \leqslant \operatorname{dim}(K)$.

\subsection{GM varieties and Lagrangian data sets}

As explained in the introduction, the constructions of Theorems 2.9 and 3.6 can be combined as follows.

Theorem 3.10. With each normal, lci polarized GM variety $(X, H)$, one can associate a canonical (functorially depending on $(X, H))$ Lagrangian data set $\left(V_{6}, V_{5}, A\right)$ and also an extended Lagrangian data set $\left(V_{6}, V_{5}, A, A_{1}\right)$ with $A_{1}$ defined up to a $\mathbb{G}_{\mathrm{m}}$-action.

Conversely, with any Lagrangian data set, one can associate a canonical (functorially depending on the data) ordinary GM intersection and also, if the field $\mathbf{k}$ is quadratically closed, a special GM intersection defined up to an isomorphism.

These two constructions are mutually inverse.

Proof. Starting with $(X, H)$, we apply Theorem 2.9 to obtain a GM data and Theorem 3.6 to obtain a Lagrangian data set $\left(V_{6}, V_{5}, A\right)$ which depends functorially on $(X, H)$. Moreover, we saw in the proof of Theorem 3.6 that the orbit of $A_{1}$ depends only on the type of $X$.

Conversely, assume that a Lagrangian data set is given. We apply Theorem 3.6 and obtain a GM data set. The choice of $\varepsilon$ involved in the construction affects $\mathbf{q}_{0}$, the even part of the family of quadrics cutting out $X$ in $\mathbf{P}(W)$, by scalar multiplication. In particular, the ordinary GM intersection associated with this data set does not depend on this choice. However, the associated special GM intersection is uniquely determined if the field is quadratically closed, but only up to isomorphism. For functoriality, note that given an isomorphism of Lagrangian data sets, one can choose $\varepsilon$ and $\varepsilon^{\prime}$ in a compatible way; the construction of Theorem 3.6 then provides an isomorphism of the corresponding GM data sets, which by Theorem 2.9 gives an isomorphism of the corresponding GM varieties.

It is clear that the constructions are mutually inverse.

Using this, one can give a criterion for GM varieties to be isomorphic and describe the automorphism group of a GM variety in terms of the associated Lagrangian data set. Consider the group

$$
\operatorname{PGL}\left(V_{6}\right)_{A, V_{5}}:=\left\{g \in \operatorname{PGL}\left(V_{6}\right) \mid\left(\bigwedge^{3} g\right)(A)=A, g\left(V_{5}\right)=V_{5}\right\}
$$

of automorphisms of $\mathbf{P}\left(V_{6}\right)$ stabilizing $A$ and $V_{5}$.

Corollary 3.11. Let $(X, H)$ and $\left(X^{\prime}, H^{\prime}\right)$ be normal, lci polarized GM varieties, with corresponding Lagrangian data sets $\left(V_{6}, V_{5}, A\right)$ and $\left(V_{6}^{\prime}, V_{5}^{\prime}, A^{\prime}\right)$.

(a) Any isomorphism $\phi$ : $(X, H) \stackrel{\sim}{\longrightarrow}\left(X^{\prime}, H^{\prime}\right)$ induces an isomorphism $V_{6} \stackrel{\sim}{\longrightarrow} V_{6}^{\prime}$ which takes $V_{5}$ to $V_{5}^{\prime}$ and $A$ to $A^{\prime}$.

(b) Conversely, if either $X$ and $X^{\prime}$ are both ordinary, or they are both special and $\mathbf{k}$ is quadratically closed, every isomorphism $V_{6} \stackrel{\sim}{\longrightarrow} V_{6}^{\prime}$ that takes $V_{5}$ to $V_{5}^{\prime}$ and $A$ to $A^{\prime}$ is induced by an isomorphism between $(X, H)$ and $\left(X^{\prime}, H^{\prime}\right)$.

$\left(\mathrm{c}^{\prime}\right)$ If $(X, H)$ is ordinary, $\operatorname{Aut}_{H}(X) \simeq \operatorname{PGL}\left(V_{6}\right)_{A, V_{5}}$. 


\section{O. Debarre and A. Kuznetsov}

$\left(\mathrm{c}^{\prime \prime}\right)$ If $(X, H)$ is special, there is an exact sequence

$$
0 \rightarrow \mathbf{Z} / 2 \rightarrow \operatorname{Aut}_{H}(X) \rightarrow \operatorname{PGL}\left(V_{6}\right)_{A, V_{5}} \rightarrow 1 .
$$

Proof. Part (a) follows from Theorem 3.10 and part (b) is also explained in the proof of that theorem. This implies that the image of the morphism $\operatorname{Aut}_{H}(X) \rightarrow \operatorname{PGL}\left(V_{6}\right)$ is $\mathrm{PGL}\left(V_{6}\right)_{A, V_{5}}$ and parts $\left(\mathrm{c}^{\prime}\right)$ and $\left(\mathrm{c}^{\prime \prime}\right)$ then follow from Corollary 2.32 .

When we use Theorem 3.10 for a normal, lci (polarized) GM variety $X$, we denote by $W, V_{6}$, $V_{5}, A, A_{1}$, etc., the associated vector spaces; to emphasize the dependence of the data on the original variety, we sometimes write $W(X), V_{6}(X), V_{5}(X), A(X), A_{1}(X)$, etc.

Conversely, given an extended Lagrangian data set $\left(V_{6}, V_{5}, A, A_{1}\right)$, we denote by $X_{A, A_{1}, V_{5}}$ the corresponding GM intersection (usually the vector space $V_{6}$ will be fixed, so we exclude it from the notation). Sometimes, we will write $X_{A, 0, V_{5}}$ for the GM intersection corresponding to $A_{1}=L$, write $X_{A, 1, V_{5}}$ for the GM intersection corresponding to the choice $A_{1} \neq L$ and $A_{1} \neq \mathbf{k}$, and write $X_{A, \infty, V_{5}}$ for the GM intersection corresponding to $A_{1}=\mathbf{k}$. The meaning of this notation is explained by the following lemma.

Lemma 3.12. Let $\left(V_{6}, V_{5}, A\right)$ be a Lagrangian data set such that $X:=X_{A, 0, V_{5}}$, with the choice $A_{1}=L$, is a $G M$ variety. Then $X$ is ordinary, $X_{A, 1, V_{5}} \simeq X_{\text {spe }}$ is the double covering of $M_{X}$ branched along $X$ (hence is a special GM variety), and $X_{A, \infty, V_{5}}$ is the cone over $X$ with vertex a point.

Proof. If $A_{1}=L$, we have $W_{1}=\operatorname{Im}\left(A_{1} \rightarrow \mathbf{k}\right)=0$; hence $X$ is ordinary. If $A_{1} \neq L$ and $A_{1} \neq \mathbf{k}$, the space $W_{1}$ is 1-dimensional and $\mathbf{q}_{1}$ induces an isomorphism $V_{6} / V_{5} \simeq \mathrm{S}^{2} W_{1}^{\vee}$. By Lemma 2.33, we have $X_{A, 1, V_{5}}=X_{\text {spe }}$.

Finally, if $A_{1}=\mathbf{k}$, the space $W_{1}$ is 1 -dimensional but $\mathbf{q}_{1}=0$. Therefore, $X_{A, \infty, V_{5}}$ is cut out in the cone over $M_{X}$ by one equation $\mathbf{q}_{0}(v)=0$ (where $v$ is any vector in $V_{6} \backslash V_{5}$ ), hence is the cone over $X$.

The construction of a GM intersection $X_{A, A_{1}, V_{5}}$ is quite involved. However, some of its geometric properties can be read off directly from $A, A_{1}$, and $V_{5}$. The next proposition explains this.

Proposition 3.13. Let $X=X_{A, A_{1}, V_{5}}$ be the $G M$ intersection associated with an lci Lagrangian data set $\left(V_{6}, V_{5}, A, A_{1}\right)$ (in particular, $A_{1} \neq \infty$ ).

(a) We have

$$
\begin{aligned}
W(X)_{0} & =A /\left(A \cap \bigwedge^{3} V_{5}\right), & W(X)_{1} & =A_{1} /\left(A_{1} \cap L\right), \\
W(X)_{0}^{\perp} & =A \cap \bigwedge^{3} V_{5}, & W(X)_{1}^{\perp} & =A_{1} \cap L .
\end{aligned}
$$

(b) For $v \in V_{6} \backslash V_{5}$, we have

$$
\operatorname{Ker}(\mathbf{q}(v))=A \cap\left(v \wedge \wedge^{2} V_{5}\right) .
$$

(c) If $X$ is a GM variety, we have

$$
\operatorname{dim}(X)= \begin{cases}5-\operatorname{dim}\left(A \cap \bigwedge^{3} V_{5}\right) & \text { if } A_{1}=0, \\ 6-\operatorname{dim}\left(A \cap \bigwedge^{3} V_{5}\right) & \text { if } A_{1}=1 .\end{cases}
$$

Proof. (a) The first line is just the definition of $W_{0}$ and $W_{1}$ in the proof of Theorem 3.6. For the second line, note that $W(X)=W(X)_{0} \oplus W(X)_{1}$ is the image of the Lagrangian subspace 


\section{GuSHEL-MuKaI VARIETIES}

$\hat{A}=A \oplus A_{1}$ by the projection to the second summand of the direct sum decomposition (3.4). Hence its annihilator is the intersection of $\hat{A}$ with the first summand.

(b) By Proposition C.1, the kernel of the quadratic form $\tilde{\mathbf{q}}(v)$ on $\hat{A}$ is the direct sum $(\hat{A} \cap$ $\left.\left(\bigwedge^{3} V_{5} \oplus L\right)\right) \oplus\left(\hat{A} \cap\left(\left(v \wedge \wedge^{2} V_{5}\right) \oplus \mathbf{k}\right)\right)$. The quadratic form $\mathbf{q}(v)$ is induced by taking the quotient with respect to the first summand; hence its kernel is the second summand. Since $A_{1} \neq \mathbf{k}$ by the lci assumption, it is equal to the right side of (3.8).

(c) If $X$ is a GM variety, we have $\operatorname{dim}(X)=\operatorname{dim}(W(X))-5$. On the other hand, by part (a), we have

$$
\operatorname{dim}\left(W(X)_{0}\right)=10-\operatorname{dim}\left(A \cap \bigwedge^{3} V_{5}\right), \quad \operatorname{dim}\left(W(X)_{1}\right)=1-\operatorname{dim}\left(A_{1} \cap L\right) .
$$

Combining these, we get (3.9).

In the next proposition, we discuss the relation between the Lagrangian data set of a GM variety $X$ and that of a hyperplane section. Such a hyperplane section is given by a linear function on the space $W(X)=W(X)_{0} \oplus W(X)_{1}$. Moreover, by Proposition 3.13(a), we have an identification $W(X)_{0}^{\vee}=\bigwedge^{3} V_{5} /\left(A \cap \bigwedge^{3} V_{5}\right)$. Any linear function on $W(X)_{0}$ can therefore be lifted to an element of $\bigwedge^{3} V_{5}$.

Proposition 3.14. Let $X$ be a normal, lci $G M$ variety, and let $\left(V_{6}, V_{5}, A\right)$ be the corresponding Lagrangian data. Let $X^{\prime} \subset X$ be a hyperplane section of $X$ which is also normal and lci, and let $\eta_{0} \in \bigwedge^{3} V_{5}$ be a lift of the even part of the equation of $X^{\prime}$. The Lagrangian data set of $X^{\prime}$ is then isomorphic to $\left(V_{6}, V_{5}, A\left(X^{\prime}\right)\right)$, where

(a) if $X^{\prime}$ has the same type as $X$, we have $A\left(X^{\prime}\right)=\left(A \cap \eta_{0}^{\perp}\right) \oplus \mathbf{k} \eta_{0}$;

(b) if $X$ is special and $X^{\prime}$ is ordinary, we have $A\left(X^{\prime}\right)=\left(A \cap \eta^{\perp}\right) \oplus \mathbf{k} \eta$ for some $\eta \in A \oplus \mathbf{k} \eta_{0}$;

(c) if $X$ is special and $X^{\prime}=X_{\text {ord }}$, we have $A\left(X^{\prime}\right)=A$.

In particular, $\operatorname{dim}\left(A \cap A\left(X^{\prime}\right)\right)=9$ unless $X$ is special and $X^{\prime}=X_{\text {ord }}$.

Proof. Let us show $A \cap \eta_{0}^{\perp} \subset A\left(X^{\prime}\right)$. Consider the commutative diagram

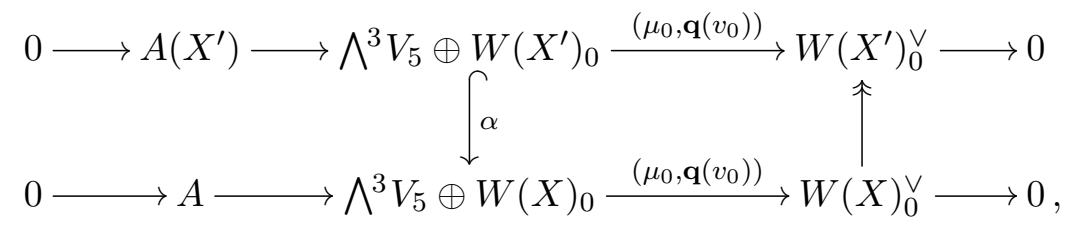

where the rows are the exact sequences (3.6) defining $A$ and $A\left(X^{\prime}\right)$, respectively, $v_{0} \in V_{6} \backslash V_{5}$ is a fixed vector, and we omit the $L$-factors for simplicity. The image of $A \cap \eta_{0}^{\perp}$ under the left bottom arrow is contained in $\bigwedge^{3} V_{5} \oplus\left(W(X)_{0} \cap \eta_{0}^{\perp}\right)$, hence in the image of $\alpha$. Therefore, by the commutativity of the diagram, it is in the kernel of the top right arrow. This proves $A \cap \eta_{0}^{\perp} \subset A\left(X^{\prime}\right)$ and in particular $\operatorname{dim}\left(A\left(X^{\prime}\right) \cap A\right) \geqslant 9$.

Any Lagrangian subspace $A^{\prime}$ containing $A \cap \eta_{0}^{\perp}$ can be written as $\left(A \cap \eta^{\perp}\right) \oplus \mathbf{k} \eta$ for some $\eta \in A \oplus \mathbf{k} \eta_{0}$. This proves part (b). Furthermore, if $X^{\prime}$ has the same type as $X$, the odd part of the equation of $X^{\prime}$ is zero, hence $\eta_{0} \in W\left(X^{\prime}\right)_{0}^{\perp}=A\left(X^{\prime}\right) \cap \bigwedge^{3} V_{5} \subset A\left(X^{\prime}\right)$. Thus $\eta_{0} \subset A\left(X^{\prime}\right)$, hence $\eta=\eta_{0}$, and part (a) follows. Finally, part (c) was already proved in Lemma 3.12.

\subsection{Strongly smooth GM varieties}

We say that a subspace $A \subset \wedge^{3} V_{6}$ contains no decomposable vectors if (see Section B.1)

$$
\mathbf{P}(A) \cap \operatorname{Gr}\left(3, V_{6}\right)=\varnothing .
$$




\section{O. Debarre And A. KuZnetsov}

The crucial Theorem 3.16 below shows, among other things, that when a Lagrangian subspace $A$ contains no decomposable vectors, the GM intersections $X_{A, A_{1}, V_{5}}$ defined at the end of Section 3.2 are smooth GM varieties for all choices of $A_{1} \neq \mathbf{k}$ and all choices of hyperplanes $V_{5} \subset V_{6}$.

Recall that for any GM intersection $X$, we defined the schemes $M_{X}=\mathrm{C}_{K} \operatorname{Gr}\left(2, V_{5}\right) \cap \mathbf{P}(W)$ and $M_{X}^{\prime}=\operatorname{Gr}\left(2, V_{5}\right) \cap \mathbf{P}\left(W_{0}\right)$ (Section 2.4).

Definition 3.15. A GM variety $X$ is strongly smooth if both intersections $X$ and $M_{X}^{\prime}$ are dimensionally transverse and smooth.

By Proposition 2.22, any smooth GM variety is strongly smooth except perhaps if it is an ordinary GM surface or an ordinary GM curve. Also note that $X$ is strongly smooth if and only if $X_{\mathrm{opp}}$ is strongly smooth (unless $X$ is a special GM curve, in which case $X_{\mathrm{opp}}=X_{\mathrm{ord}}$ is just not defined).

Theorem 3.16. Assume $\mathbf{k}=\mathbf{C}$. Let $\left(V_{6}, V_{5}, A, A_{1}\right)$ be an extended Lagrangian data set with $A_{1} \neq \infty$, and let $X:=X_{A, A_{1}, V_{5}}$ be the corresponding GM intersection. The following conditions are equivalent:

(a) The GM intersection $X$ is a strongly smooth $G M$ variety.

(b) We have $\operatorname{dim}\left(A \cap \bigwedge^{3} V_{5}\right)+\operatorname{dim}\left(A_{1} \cap L\right) \leqslant 5$, the decomposable vectors in $A$ are all in $\bigwedge^{3} V_{5}$, and $\mathbf{P}\left(A \cap \bigwedge^{3} V_{5}\right) \cap \operatorname{Gr}\left(3, V_{5}\right)$ is a smooth, dimensionally transverse intersection in $\mathbf{P}\left(\bigwedge^{3} V_{5}\right)$.

In particular, if $\operatorname{dim}\left(A \cap \bigwedge^{3} V_{5}\right) \leqslant 3$, the GM intersection $X$ is a smooth GM variety if and only if $A$ contains no decomposable vectors.

Proof. Assume that condition (b) holds. By Proposition 3.13, we have $W_{0}^{\perp}=A \cap \bigwedge^{3} V_{5}$, and $\mathbf{P}\left(W_{0}^{\perp}\right) \cap \mathrm{Gr}\left(3, V_{5}\right)$ is a smooth, dimensionally transverse intersection. By Proposition 2.24, so is $M_{X}^{\prime}$. Now, assume, to obtain a contradiction, that for $v \in V_{6} \backslash V_{5}$, the GM intersection $X_{A, A_{1}, V_{5}}=\mathrm{C}_{\operatorname{Ker}(\mu)} M_{X}^{\prime} \cap Q(v)$ is not dimensionally transverse or is singular. In both cases, there exists a point $w \in \mathrm{C}_{\operatorname{Ker}(\mu)} M_{X}^{\prime} \cap Q(v)$ such that the tangent space $\mathbf{T}_{Q(v), w}$ contains $\mathbf{T}_{M_{X}^{\prime}, w}$.

As $A_{1} \neq \mathbf{k}$, we know by the proof of Lemma 3.12 that $w$ cannot be the vertex of the cone. Since $M_{X}^{\prime}$ is smooth, $\mathbf{T}_{M_{X}^{\prime}, w}$ is therefore the intersection of the tangent spaces to Plücker quadrics; hence $\mathbf{T}_{Q(v), w}$ coincides with $\mathbf{T}_{Q\left(v^{\prime}\right), w}$ for some $v^{\prime} \in V_{5}$. Therefore, for some $t \in \mathbf{k}$, the quadric $Q\left(v+t v^{\prime}\right)$ is singular at $w$ and, replacing $v$ with $v+t v^{\prime}$, we may assume that $w$ is in the kernel of $\mathbf{q}(v)$. By (3.8), this kernel is $A \cap\left(v \wedge \wedge^{2} V_{5}\right)$; therefore, $v \wedge \mu(w) \in A$. Since $\mu(w) \in M_{X}^{\prime}$, we have $\mu(w) \in \operatorname{Gr}\left(2, V_{5}\right)$, so $v \wedge \mu(w)$ is a decomposable vector in $A$ which is not in $\bigwedge^{3} V_{5}$, contradicting condition (b). Therefore, $X_{A, A_{1}, V_{5}}$ is a smooth GM variety (its dimension is at least 1 because of the condition $\left.\operatorname{dim}\left(A \cap \bigwedge^{3} V_{5}\right)+\operatorname{dim}\left(A_{1} \cap L\right) \leqslant 5\right)$.

Conversely, assume that condition (a) holds. Since $\operatorname{dim}(X) \geqslant 1$, we have $\operatorname{dim}\left(A \cap \bigwedge^{3} V_{5}\right)+$ $\operatorname{dim}\left(A_{1} \cap L\right) \leqslant 5$ by Proposition 3.13. Assume, again to obtain a contradiction, that $A$ contains a decomposable vector not in $\wedge^{3} V_{5}$. This vector can be written as $v \wedge v_{1} \wedge v_{2}$, where $v \in V_{6} \backslash V_{5}$ and $v_{1}, v_{2} \in V_{5}$. As this vector is both in $A$ and in $v \wedge \wedge^{2} V_{5}$, it is by (3.8) in the kernel of $\mathbf{q}(v)$. On the other hand, $v_{1} \wedge v_{2} \in \operatorname{Gr}\left(2, V_{5}\right)$ and, as $\lambda_{3}\left(v \wedge v_{1} \wedge v_{2}\right)=\lambda(v) v_{1} \wedge v_{2}$, it is also in $\mathbf{P}\left(W_{0}\right)$. So $v_{1} \wedge v_{2}$ is in $M_{X}^{\prime}$ and is a singular point of the quadric $Q(v)$. Hence it is a singular point of $X=\mathrm{C}_{\operatorname{Ker}(\mu)} M_{X}^{\prime} \cap Q(v)$, contradicting condition (a). Finally, $\mathbf{P}\left(A \cap \bigwedge^{3} V_{5}\right) \cap \mathrm{Gr}\left(3, V_{5}\right)$ is a smooth, dimensionally transverse intersection by Proposition 2.24 , since $M_{X}^{\prime}=\mathbf{P}\left(\left(A \cap \bigwedge^{3} V_{5}\right)^{\perp}\right) \cap \operatorname{Gr}\left(2, V_{5}\right)$ is so by the definition of strong smoothness.

When $\operatorname{dim}\left(X_{A, A_{1}, V_{5}}\right) \geqslant 3$, smoothness is equivalent to strong smoothness by Proposition 2.22 and $\operatorname{dim}\left(A \cap \bigwedge^{3} V_{5}\right) \leqslant 3$ by (3.9); hence the intersection $\mathbf{P}\left(A \cap \bigwedge^{3} V_{5}\right) \cap \operatorname{Gr}\left(3, V_{5}\right)$ is dimensionally 


\section{Gushel-Mukai VARIETIES}

transverse if and only if it is empty. Condition (b) is therefore equivalent to the absence of decomposable vectors in $A$.

Remark 3.17. Let $X$ be a strongly smooth complex GM variety, with associated Lagrangian subspace $A=A(X)$. Set $\ell:=\operatorname{dim}\left(A \cap \bigwedge^{3} V_{5}\right)$. Formula (3.9) then reads

$$
\ell= \begin{cases}5-\operatorname{dim}(X) & \text { if } X \text { is ordinary } \\ 6-\operatorname{dim}(X) & \text { if } X \text { is special }\end{cases}
$$

Theorem 3.16 implies the following:

- If $\ell \leqslant 3$ (that is, $\operatorname{dim}(X) \geqslant 3$ or $X$ is an ordinary GM surface), $A$ contains no decomposable vectors. By Theorem B.2, the condition $\operatorname{dim}\left(A \cap \bigwedge^{3} V_{5}^{\prime}\right) \leqslant 3$ holds for all hyperplanes $V_{5}^{\prime} \subset V_{6}$.

- If $\ell=4$ (that is, $X$ is an ordinary GM curve or a special GM surface), $A$ contains exactly five decomposable vectors and they are all in $\mathbf{P}\left(\bigwedge^{3} V_{5}\right)$.

- If $\ell=5$ (that is, $X$ is a special (bielliptic) curve), the decomposable vectors in $\mathbf{P}(A)$ form a smooth elliptic quintic curve contained in $\mathbf{P}\left(\bigwedge^{3} V_{5}\right)$.

\subsection{EPW sextics}

For a GM variety $X$, we defined in Section 3.1 the discriminant locus $\operatorname{Disc}(X) \subset \mathbf{P}\left(V_{6}\right)$, which is either a sextic hypersurface or the whole space. We now give a unified proof of a generalization of Theorem 3.1 of Iliev and Manivel, proving that the schemes $\operatorname{Disc}(X)$ and $Y_{A(X)}$ are equal.

Proposition 3.18. Let $X$ be a normal, lci, polarized $G M$ variety, and let $\left(V_{6}, V_{5}, A\right)$ be the corresponding Lagrangian data. If either $Y_{A}$ or $\operatorname{Disc}(X)$ is a reduced hypersurface, we have $Y_{A}=\operatorname{Disc}(X)$.

Proof. We have $\operatorname{Ker}(\mathbf{q}(v))=A \cap\left(v \wedge \wedge^{2} V_{5}\right)$ by (3.8); hence, by the definition of $Y_{A}$ (see Definition B.1), we see that $Y_{A}$ and $\operatorname{Disc}\left(X_{A, A_{1}, V_{5}}\right)$ coincide as sets on the complement of $\mathbf{P}\left(V_{5}\right)$. On the other hand, each of $Y_{A}$ and $\operatorname{Disc}\left(X_{A, A_{1}, V_{5}}\right)$ is either a sextic hypersurface or $\mathbf{P}\left(V_{6}\right)$. If one is a reduced hypersurface, so is the other, and they are equal.

The following result was proved in [OGr12, Proposition 2.18] when $X$ is a smooth ordinary fivefold (it is then automatically strongly smooth).

Corollary 3.19. Let $X$ be a strongly smooth complex GM variety. The subschemes $Y_{A(X)}$ and $\operatorname{Disc}(X)$ of $\mathbf{P}\left(V_{6}\right)$ are equal, and they are reduced sextic hypersurfaces. They are also integral and normal unless $X$ is a bielliptic curve, in which case they are the union of a hyperplane and an integral quintic hypersurface.

Proof. As in Remark 3.17, let us set $\ell:=\operatorname{dim}\left(A(X) \cap \bigwedge^{3} V_{5}\right)$ (this is $5-\operatorname{dim}(X)$ if $X$ is ordinary, and $6-\operatorname{dim}(X)$ if $X$ is special).

If $\ell \leqslant 3$, we saw in Remark 3.17 that $A(X)$ contains no decomposable vectors; hence $Y_{A(X)}$ is an integral, normal sextic hypersurface whose singularities are described in Theorem B.2. By Proposition 3.18, it is equal to $\operatorname{Disc}(X)$.

If $\ell=4$ and $X$ is an ordinary GM curve, $\operatorname{Disc}(X)$ is a reduced hypersurface (Proposition 3.2). By Proposition 3.18 again, it is equal to $Y_{A(X)}$. If $X$ is a special GM surface then, by Lemma 3.3, its discriminant locus equals $\operatorname{Disc}\left(X_{\text {ord }}\right)$, which is an ordinary GM curve, and the previous argument applies.

Finally, if $\ell=5$, then $X$ is a special (that is, bielliptic) GM curve, and the statements follow from Proposition 3.2 in the same way. 


\section{O. Debarre and A. Kuznetsov}

The EPW sequence $Y_{A^{\perp}}^{\geqslant l}$ in $\mathbf{P}\left(V_{6}^{\vee}\right)$ is defined in (B.4).

Remark 3.20. Let $X$ be a strongly smooth complex GM curve, with Plücker hyperplane $V_{5}:=$ $V_{5}(X) \subset V_{6}$ and associated Lagrangian $A=A(X)$.

(1) When $X$ is ordinary, $V_{5}$ is a point of $Y_{A^{\perp}}^{\geqslant 4}$ by Remark 3.17 and it is the only point. Indeed, $\mathbf{P}(A)$ contains exactly five decomposable vectors $\wedge^{3} V_{3,1}, \ldots, \wedge^{3} V_{3,5}$, which are all in $\bigwedge^{3} V_{5}$ (Remark 3.17). Moreover, by [AH81, (5.10)] or [OGr15, (2.3.2)], we have $V_{5}=V_{3, i}+V_{3, j}$ for all $i \neq j$. Assume that $V_{5}^{\prime} \subset V_{6}$ is another hyperplane in $Y_{A^{\perp}}^{\geqslant 4}$, so that $\operatorname{dim}\left(A \cap \bigwedge^{3} V_{5}^{\prime}\right) \geqslant 4$. The points of the scheme $M^{\prime}:=\mathbf{P}\left(A \cap \bigwedge^{3} V_{5}^{\prime}\right) \cap \operatorname{Gr}\left(3, V_{5}^{\prime}\right)$ must be among $V_{3,1}, \ldots, V_{3,5}$. This implies $V_{5}^{\prime}=V_{5}$ unless $M^{\prime}$ is a single point, say $w:=V_{3,1}$, with multiplicity 5 . Since $M^{\prime}$ is a dimensionally transverse intersection, one checks, using its Koszul resolution in $\operatorname{Gr}\left(3, V_{5}^{\prime}\right)$ and Kodaira vanishing, that its linear span is $\mathbf{P}\left(A \cap \bigwedge^{3} V_{5}^{\prime}\right)$. This implies $\mathbf{P}\left(A \cap \bigwedge^{3} V_{5}^{\prime}\right) \subset \mathbf{T}_{\mathrm{Gr}\left(3, V_{5}^{\prime}\right), w}$. But the 3-plane $\mathbf{P}\left(A \cap \bigwedge^{3} V_{5}^{\prime}\right)$ must then meet the 4-dimensional Schubert cycle $\operatorname{Gr}\left(3, V_{5}^{\prime}\right) \cap \mathbf{T}_{\mathrm{Gr}\left(3, V_{5}^{\prime}\right), w}=\mathrm{C}\left(\mathbf{P}^{2} \times \mathbf{P}^{1}\right)$ along a curve, which consists of decomposable vectors in $\mathbf{P}(A)$. This gives a contradiction.

(2) When $X$ is special (that is, bielliptic), $V_{5}$ is the only point of $Y_{A^{\perp}}^{\geqslant 5}$. Indeed, the set $\Theta_{A}$ of decomposable vectors in $\mathbf{P}(A)$ is a smooth elliptic curve contained in $\mathbf{P}\left(\bigwedge^{3} V_{5}\right)$ (Remark 3.17). If $V_{5}^{\prime} \subset V_{6}$ is another point of $Y_{A^{\perp}}^{\geqslant 5}$, the scheme $\mathbf{P}\left(A \cap \wedge^{3} V_{5}^{\prime}\right) \cap \operatorname{Gr}\left(3, V_{5}^{\prime}\right)$ has everywhere dimension at least 1 and is contained in $\Theta_{A}$. These two schemes are thus equal, hence contained in $\operatorname{Gr}\left(3, V_{5}\right) \cap$ $\operatorname{Gr}\left(3, V_{5}^{\prime}\right)=\operatorname{Gr}\left(3, V_{5} \cap V_{5}^{\prime}\right)$. Since the linear span of $\Theta_{A}$ has dimension 4, this implies $V_{5}=V_{5}^{\prime}$.

By Corollary 3.11(b), the curve $X$ is determined (up to isomorphism) by $A(X)$ and the hyperplane $V_{5} \subset V_{6}$. In both cases, it is therefore uniquely determined by $A(X)$. In fact, $X$ is also uniquely determined (up to isomorphism) by the sextic $Y_{A(X)}$. When $X$ is special, this is because $\mathbf{P}\left(V_{5}\right)$ is the only degree 1 component of $Y_{A(X)}$ (Propositions 3.2 and 3.18). When $X$ is ordinary, the singular locus of $Y_{A(X)}$ is the union of five planes (one for each decomposable vector in $A$; see Remark 3.17 and (B.3)), an integral surface $S$ coming from the singular locus of the theta divisor of the Jacobian of $X$ (see [AH81, Theorem 1.4]; one checks that it has degree 30 and is contained in $Y_{A(X)}^{\geqslant 2}$, and another irreducible surface of degree 10, also contained in $Y_{A(X)}^{\geqslant 2}$. Moreover, the intersection of $S$ with each of these five planes is a plane sextic curve whose normalization is $X$ (this follows from [AH81, Section 5, footnote 1]).

When $\mathbf{k}=\mathbf{C}$, we can restate the results of Corollary 3.11 in terms of the EPW sextic $Y_{A(X)}$. The group PGL $\left(V_{6}\right)_{A, V_{5}}$ was defined in (3.7); we also define

$$
\operatorname{PGL}\left(V_{6}\right)_{Y_{A}, V_{5}}:=\left\{g \in \operatorname{PGL}\left(V_{6}\right) \mid g\left(Y_{A}\right)=Y_{A}, g\left(V_{5}\right)=V_{5}\right\} .
$$

Proposition 3.21. Let $(X, H)$ and $\left(X^{\prime}, H^{\prime}\right)$ be normal lci complex polarized $G M$ varieties, with corresponding Lagrangian data sets $\left(V_{6}, V_{5}, A\right)$ and $\left(V_{6}^{\prime}, V_{5}^{\prime}, A^{\prime}\right)$.

(a) Any isomorphism $\phi:(X, H) \stackrel{\sim}{\longrightarrow}\left(X^{\prime}, H^{\prime}\right)$ induces an isomorphism $V_{6} \stackrel{\sim}{\longrightarrow} V_{6}^{\prime}$ which takes $V_{5}$ to $V_{5}^{\prime}$ and $Y_{A}$ to $Y_{A^{\prime}}$.

(b) If $X$ is smooth and $X$ and $X^{\prime}$ have same type and same dimension $n \geqslant 3$, every isomorphism $\varphi: V_{6} \stackrel{\sim}{\longrightarrow} V_{6}^{\prime}$ that takes $V_{5}$ to $V_{5}^{\prime}$ and $Y_{A}$ to $Y_{A^{\prime}}$ is induced by an isomorphism between $(X, H)$ and $\left(X^{\prime}, H^{\prime}\right)$.

(c) If $X$ is smooth of dimension $n \geqslant 3$, we have $\operatorname{PGL}\left(V_{6}\right)_{A, V_{5}}=\operatorname{PGL}\left(V_{6}\right)_{Y_{A}, V_{5}}$ and the group $\operatorname{Aut}(X)$ is finite.

(d) If $X$ is smooth of dimension $n \geqslant 3$, the group $\operatorname{Aut}(X)$ is trivial if $X$ is very general ordinary and $\operatorname{Aut}(X) \simeq \mathbf{Z} / 2$ if $X$ is very general special.

Proof. (a) This follows from Corollary 3.11 and the definition of $Y_{A}$. 


\section{Gushel-Mukai VARIETIES}

(b) By Theorem 3.16, the space $A$ contains no decomposable vectors, while by Proposition B.8(b), we have $\left(\bigwedge^{3} \varphi\right)(A)=A^{\prime}$. Item (b) then follows from Corollary 3.11.

(c) The equality $\operatorname{PGL}\left(V_{6}\right)_{A, V_{5}}=\operatorname{PGL}\left(V_{6}\right)_{Y_{A}, V_{5}}$ is proved by the argument in part (b). Furthermore, $\operatorname{Aut}_{H}(X)=\operatorname{Aut}(X)$ when $X$ is smooth and $\operatorname{dim}(X) \geqslant 3$ (Lemma 2.29). Therefore, to show that $\operatorname{Aut}(X)$ is finite, it is enough by Corollary 3.11(c) to show that $\operatorname{PGL}\left(V_{6}\right)_{A, V_{5}}$ is finite. But the latter is a subgroup of the group $\operatorname{PGL}\left(V_{6}\right)_{A}$ defined in (B.9), and that group is finite by Proposition B.9(a).

(d) It is enough to show that $\operatorname{PGL}\left(V_{6}\right)_{A}=\operatorname{Aut}\left(Y_{A}\right)$ is trivial for a very general Lagrangian subspace $A \subset \wedge^{3} V_{6}$. This is Proposition B.9(b).

Lengthy computations also show directly that for any smooth complex GM variety $X$ of dimension at least 3 , one has $H^{0}\left(X, T_{X}\right)=0$ (these computations were done in [DIM12, Theorem 3.4] for ordinary GM threefolds and in [DIM15, Proposition 4.1] for GM fourfolds). We recover the fact that the automorphism group of $X$, being discrete, is finite, as asserted in the proposition.

\subsection{Period partners}

As we saw in the previous section, many properties of a GM variety depend only on $A(X)$, the even part of the corresponding Lagrangian subspace. More evidence will come further on.

Definition 3.22. Normal, lci GM varieties $X_{1}$ and $X_{2}$ are period partners if $\operatorname{dim}\left(X_{1}\right)=\operatorname{dim}\left(X_{2}\right)$ and there exists an isomorphism $\varphi: V_{6}\left(X_{1}\right) \stackrel{\sim}{\longrightarrow} V_{6}\left(X_{2}\right)$ such that $\left(\bigwedge^{3} \varphi\right)\left(A\left(X_{1}\right)\right)=A\left(X_{2}\right)$.

Remark 3.23. The last condition in the definition implies $\varphi\left(Y_{A\left(X_{1}\right)}\right)=Y_{A\left(X_{2}\right)}$. Conversely, assume that the lci GM varieties $X_{1}$ and $X_{2}$ are such that $\operatorname{dim}\left(X_{1}\right)=\operatorname{dim}\left(X_{2}\right) \geqslant 3$, that there exists an isomorphism $\varphi: V_{6}\left(X_{1}\right) \stackrel{\sim}{\sim} V_{6}\left(X_{2}\right)$ such that $\varphi\left(Y_{A\left(X_{1}\right)}\right)=Y_{A\left(X_{2}\right)}$, and that $X_{1}$ is smooth. Proposition B.8 then shows that $X_{1}$ and $X_{2}$ are period partners and that $X_{2}$ is also smooth by Theorem 3.16.

Period partners may have different types; an ordinary GM variety may be a period partner of a special GM variety.

Remark 3.24. The name "period partners" suggests a relation with the period map. In the article [DK16], we show that, indeed, smooth GM varieties of the same dimension are period partners if and only if they are in the same fiber of an appropriately defined period map from the moduli space of GM varieties to an appropriate period domain.

A major difficulty in realizing the program of the remark is the construction of the moduli space of GM varieties. While this space is not available, we can formulate a naive description of the fiber of the period map as follows. The dual EPW stratification is defined in (B.4) and the group $\operatorname{PGL}\left(V_{6}\right)_{A}$ is defined in (B.9).

Theorem 3.25. Let $X$ be a smooth GM variety of dimension $n \geqslant 3$ defined over a quadratically closed field, with corresponding Lagrangian data $\left(V_{6}, V_{5}, A\right)$. There is a natural bijection (defined in the proof) between the set of isomorphism classes of period partners of $X$ and the set $\left(Y_{A^{\perp}}^{5-n} \sqcup Y_{A^{\perp}}^{6-n}\right) / \operatorname{PGL}\left(V_{6}\right)_{A}$.

This theorem gives a precise answer to the question asked at the end of [IM11, Section 4.5]. 


\section{O. Debarre and A. Kuznetsov}

Proof. By Proposition 3.21, an lci GM variety $X^{\prime}$ is a period partner of $X$ if and only if there is an isomorphism $\varphi: V_{6}\left(X^{\prime}\right) \stackrel{\sim}{\longrightarrow} V_{6}$ such that $\left(\bigwedge^{3} \varphi\right)\left(A\left(X^{\prime}\right)\right)=A$ and $\varphi\left(V_{5}\left(X^{\prime}\right)\right) \in Y_{A^{\perp}}^{5-n}$ if $X^{\prime}$ is ordinary, or $\varphi\left(V_{5}\left(X^{\prime}\right)\right) \in Y_{A^{\perp}}^{6-n}$ if $X^{\prime}$ is special (by (3.9), this ensures the equality of the dimensions). Since such an $X^{\prime}$ is automatically a smooth GM variety by Theorem 3.16, and the isomorphism $\varphi$ is defined up to the action of $\operatorname{PGL}\left(V_{6}\right)_{A}$, this proves the lemma.

In Section 4, we show that over $\mathbf{C}$, period partners are always birationally isomorphic. In [DK16, Theorem 5.1], we show that they have the same primitive Hodge structure in the middle cohomology (in case of even dimensions). Finally, in the joint work [KP16] of the second author with Alexander Perry, we discuss a relation between derived categories of period partners.

\subsection{Duality}

We introduce here a notion of duality for GM varieties. It is similar in flavor to the notion of period partnership, and we will see later that dual varieties share many geometric properties, as do period partners.

Definition 3.26. Normal, lci GM varieties $X$ and $X^{\prime}$ are called dual if $\operatorname{dim}(X)=\operatorname{dim}\left(X^{\prime}\right)$ and there exists an isomorphism $\varphi: V_{6}(X) \stackrel{\sim}{\longrightarrow} V_{6}\left(X^{\prime}\right)^{\vee}$ such that $\left(\bigwedge^{3} \varphi\right)(A(X))=A\left(X^{\prime}\right)^{\perp}$.

The last condition in the definition implies $\varphi\left(Y_{A(X)}\right)=Y_{A\left(X^{\prime}\right)^{\perp}}$. Conversely, as in Remark 3.23, if $\operatorname{dim}(X)=\operatorname{dim}\left(X^{\prime}\right) \geqslant 3$, if there exists an isomorphism $\varphi: V_{6}(X) \stackrel{\sim}{\longrightarrow} V_{6}\left(X^{\prime}\right)^{\vee}$ such that $\varphi\left(Y_{A(X)}\right)=Y_{A\left(X^{\prime}\right)^{\perp}}$, and if $X$ is smooth, then $X^{\prime}$ is also smooth and dual to $X$.

Duality is a symmetric relation. Moreover, if $X^{\prime}$ and $X^{\prime \prime}$ are both dual to $X$, then they are period partners. Analogously, if $X^{\prime}$ is dual to $X$ and $X^{\prime \prime}$ is a period partner of $X^{\prime}$, then $X^{\prime \prime}$ is dual to $X$.

One can give a description of all possible duals of a given GM variety analogous to the description of the set of all period partners.

TheOREM 3.27. Let $X$ be a smooth $G M$ variety of dimension $n \geqslant 3$ defined over a quadratically closed field, with corresponding Lagrangian data $\left(V_{6}, V_{5}, A\right)$. There is a natural bijection between the set of isomorphism classes of its dual varieties and the set $\left(Y_{A}^{5-n} \sqcup Y_{A}^{6-n}\right) / \operatorname{PGL}\left(V_{6}\right)_{A}$.

Proof. The proof is identical to that of Theorem 3.25.

Let $X$ and $X^{\prime}$ be dual smooth GM varieties. Set $V_{6}:=V_{6}(X)$, and choose an isomorphism $V_{6}\left(X^{\prime}\right) \simeq V_{6}^{\vee}$ such that $A\left(X^{\prime}\right)$ corresponds to $A(X)^{\perp}$. The hyperplane $V_{5}:=V_{5}(X) \subset V_{6}$ gives a line $V_{5}^{\perp} \subset V_{6}^{\vee}$, and, conversely, the hyperplane $V_{5}^{\prime}:=V_{5}\left(X^{\prime}\right) \subset V_{6}^{\vee}$ gives a line $V_{5}^{\prime \perp} \subset V_{6}$. Since $V_{6}$ parameterizes quadrics cutting out $X$ and $V_{6}^{\vee}$ parameterizes those cutting out $X^{\prime}$, these lines define quadrics

$$
Q(X) \subset \mathbf{P}\left(W\left(X^{\prime}\right)\right) \subset \mathbf{P}\left(\bigwedge^{2} V_{5}^{\prime} \oplus \mathbf{k}\right) \quad \text { and } \quad Q\left(X^{\prime}\right) \subset \mathbf{P}(W(X)) \subset \mathbf{P}\left(\bigwedge^{2} V_{5} \oplus \mathbf{k}\right)
$$

Also consider the quadrics

$$
Q_{0}(X):=Q(X) \cap \mathbf{P}\left(\bigwedge^{2} V_{5}^{\prime}\right) \subset \mathbf{P}\left(\bigwedge^{2} V_{5}^{\prime}\right) \quad \text { and } \quad Q_{0}\left(X^{\prime}\right):=Q\left(X^{\prime}\right) \cap \mathbf{P}\left(\bigwedge^{2} V_{5}\right) \subset \mathbf{P}\left(\bigwedge^{2} V_{5}\right) .
$$

Proposition 3.28. In the above setup, assume that the line $V_{5}^{\prime \perp}$ is not contained in the hyperplane $V_{5} \subset V_{6}$. Then the duality between $V_{6}$ and $V_{6}^{\vee}$ induces a duality between the spaces $V_{5}^{\prime}$ and $V_{5}$ under which the quadrics $Q_{0}(X)$ and $Q_{0}\left(X^{\prime}\right)$ are projectively dual. 


\section{Gushel-Mukai VARIETIES}

Proof. The first statement is obvious. For the second, choose generators $v \in V_{5}^{\perp \perp} \subset V_{6}$ and $v^{\prime} \in V_{5}^{\perp} \subset V_{6}^{\vee}$. By assumption, $v \notin V_{5}$ and $v^{\prime} \notin V_{5}^{\prime}$. Consider the direct sum decomposition

$$
\mathbb{V}=\left(\bigwedge^{3} V_{5} \oplus L\right) \oplus\left(\left(v \wedge \bigwedge^{2} V_{5}\right) \oplus \mathbf{k}\right)
$$

The quadric $Q\left(X^{\prime}\right)=Q(v)$ is the quadric corresponding to the Lagrangian subspace $\hat{A} \subset \mathbb{V}$ via Proposition C.1, denoted there by $Q_{2}^{\hat{A}}$. By Lemma C.3, the quadric $Q_{0}\left(X^{\prime}\right)$ is the result of its isotropic reduction with respect to the isotropic subspace $L \subset \mathbb{V}$. Therefore, $Q_{0}\left(X^{\prime}\right)$ is the quadric corresponding to the Lagrangian subspace $A \subset \mathbb{V}_{0}=\bigwedge^{3} V_{6}$ with respect to the direct sum decomposition

$$
\bigwedge^{3} V_{6}=\bigwedge^{3} V_{5} \oplus\left(v \wedge \bigwedge^{2} V_{5}\right)
$$

that is, $Q_{0}\left(X^{\prime}\right)=Q_{2}^{A}$. The same argument shows $Q_{0}(X)=Q_{2}^{A^{\perp}}$ for the direct sum decomposition

$$
\bigwedge^{3} V_{6}^{\vee}=\bigwedge^{3} V_{5}^{\prime} \oplus\left(v^{\prime} \wedge \bigwedge^{2} V_{5}^{\prime}\right) \text {. }
$$

Finally, note that the isomorphism $\bigwedge^{3} V_{6} \stackrel{\sim}{\longrightarrow} \bigwedge^{3} V_{6}^{\vee}$ given by the symplectic form interchanges the summands of these decompositions. To prove this, since the summands are Lagrangian, it is enough to show that $v^{\prime} \wedge \wedge^{2} V_{5}^{\prime}$ annihilates $\bigwedge^{3} V_{5}$ and that $v \wedge \Lambda^{2} V_{5}$ annihilates $\bigwedge^{3} V_{5}^{\prime}$. This follows from the fact that $v^{\prime}$ annihilates $V_{5}$ (by its definition) and that $v$ annihilates $V_{5}^{\prime}$.

Since $X$ and $X^{\prime}$ are dual, this isomorphism also takes $A$ to $A^{\perp}$, hence identifies $Q_{2}^{A^{\perp}}$ with $Q_{1}^{A} \subset \mathbf{P}\left(\bigwedge^{3} V_{5}\right)$. The proposition follows, since the quadrics $Q_{1}^{A}$ and $Q_{2}^{A}$ are projectively dual by Proposition C.1.

If $\mathbf{k}$ is quadratically closed, the variety $X$ is determined by the quadric $Q_{0}\left(X^{\prime}\right)$ : if $X$ is ordinary, we have $X=\operatorname{Gr}\left(2, V_{5}\right) \cap Q_{0}\left(X^{\prime}\right)$, and if $X$ is special, $X=\left(\operatorname{Gr}\left(2, V_{5}\right) \cap Q_{0}\left(X^{\prime}\right)\right)^{\text {spe }}$. Analogously, $X^{\prime}$ is determined by the quadric $Q_{0}(X)$. Proposition 3.28 thus shows that projective duality of quadrics governs duality of GM varieties.

Remark 3.29. One can also show that if $X$ and $X^{\prime}$ are both special GM varieties, the duality between $V_{5}$ and $V_{5}^{\prime}$ can be extended to a duality between $\Lambda^{2} V_{5} \oplus \mathbf{k}$ and $\bigwedge^{2} V_{5}^{\prime} \oplus \mathbf{k}$ in such a way that the quadric $Q\left(X^{\prime}\right) \subset \mathbf{P}\left(\bigwedge^{2} V_{5} \oplus \mathbf{k}\right)$ is projectively dual to the quadric $Q(X) \subset \mathbf{P}\left(\bigwedge^{2} V_{5}^{\prime} \oplus \mathbf{k}\right)$.

\section{Rationality and birationalities}

The goal of this section is to prove that smooth complex GM varieties $X_{1}$ and $X_{2}$ of dimension at least 3 which are either period partners in the sense of Definition 3.22 (so that the Lagrangians $A\left(X_{1}\right)$ and $A\left(X_{2}\right)$ are isomorphic) or dual in the sense of Definition 3.26 (so that $A\left(X_{2}\right)$ is isomorphic to $\left.A\left(X_{1}\right)^{\perp}\right)$ are birationally isomorphic.

The proof is based on the construction of two quadric fibrations. Although all dimensions could be treated with our methods, we prefer, for the sake of simplicity, to first prove that all smooth complex GM varieties of dimensions 5 and 6 are rational, and then concentrate on the cases of dimensions 3 and 4 , for which birationality is really meaningful.

\subsection{The rationality of smooth, complex GM varieties of dimensions 5 and 6}

The rationality of a general complex GM fivefold is explained in [Rot49, Section 5]. We give a different argument, showing that all smooth GM fivefolds and sixfolds are rational.

Lemma 4.1. Any smooth complex GM variety of dimension 5 or 6 contains a smooth quintic del Pezzo surface. 


\section{O. Debarre And A. KuZnetsov}

Proof. Let $\left(V_{6}, V_{5}, A\right)$ be a Lagrangian data set corresponding to such a variety $X$, and let $Y_{A} \subset \mathbf{P}\left(V_{6}\right)$ be the corresponding EPW sextic. By Theorem 3.16, the space $A$ contains no decomposable vectors. By Lemma B.6, there is a point $v \in Y_{A}^{2} \backslash \mathbf{P}\left(V_{5}\right)$, and by (3.8), the corresponding quadric $Q(v) \subset \mathbf{P}(W)$ has corank 2 and $X=\operatorname{CGr}\left(2, V_{5}\right) \cap Q(v)$.

Let $n:=\operatorname{dim}(X) \in\{5,6\}$. The quadric $Q(v)$ has rank $n+3$ in $\mathbf{P}^{n+4}$, hence has an isotropic subspace $I \subset W$ of dimension $2+\lfloor(n+3) / 2\rfloor=6$. Then $\mathbf{P}(I) \cap X=\mathbf{P}(I) \cap \operatorname{CGr}\left(2, V_{5}\right)$ is a quintic del Pezzo surface if the intersection is dimensionally transverse and smooth. We show that this is true for a general choice of $I$.

The space of all $Q(v)$-isotropic 6-subspaces is $\operatorname{OGr}(4, n+3)$, a smooth variety which we denote by $B$. Let $\mathscr{I} \subset W \otimes \mathscr{O}_{B}$ be the tautological bundle of 6-dimensional $Q(v)$-isotropic subspaces over $B$, and let $\mathbf{P}_{B}(\mathscr{I})$ be its projectivization. It comes with a natural map $\mathbf{P}_{B}(\mathscr{I}) \rightarrow$ $\mathbf{P}\left(\bigwedge^{2} V_{5} \oplus \mathbf{k}\right)$ induced by the embedding $\mathscr{I} \subset W \otimes \mathscr{O}_{B} \subset\left(\bigwedge^{2} V_{5} \oplus \mathbf{k}\right) \otimes \mathscr{O}_{B}$. The scheme

$$
\mathscr{X}=\mathbf{P}_{B}(\mathscr{I}) \times_{\mathbf{P}\left(\bigwedge^{2} V_{5} \oplus \mathbf{k}\right)} \operatorname{CGr}\left(2, V_{5}\right)
$$

maps to $B$, and the fibers are the intersections $\mathbf{P}(I) \cap X$.

To show that the general fiber is dimensionally transverse and smooth, it is enough to show that $\mathscr{X}$ is itself smooth of the expected dimension. For this, consider the second projection $\mathscr{X} \rightarrow \operatorname{CGr}\left(2, V_{5}\right)$. Since the fibers of $\mathbf{P}_{B}(\mathscr{I})$ over $B$ are contained in $Q(v)$, the image of $\mathscr{X}$ is contained in $\operatorname{CGr}\left(2, V_{5}\right) \cap Q(v)=X$. Moreover, the fiber of this projection over a point $w \in X \subset$ $\mathbf{P}(W)$ is the space of all $Q(v)$-isotropic 6-subspaces $I \subset W$ such that $w \in I$.

Since $w$ cannot be in the kernel of $Q(v)$ (otherwise, $X$ would be singular), the space of such $I$ is parameterized by $\operatorname{OGr}(3, n+1)$. All fibers of the projection $\mathscr{X} \rightarrow X$ are therefore smooth of the same dimension; hence $\mathscr{X}$ is smooth. As observed earlier, the general fiber of the projection $\mathscr{X} \rightarrow B$ is therefore a smooth quintic del Pezzo surface.

We can now prove the rationality of smooth GM fivefolds and sixfolds.

Proposition 4.2. Any smooth complex GM variety of dimension 5 or 6 is rational.

Proof. We use the notation of the previous proof. Let $Q=Q(v)$ be a quadric of corank 2 in $\mathbf{P}(W)$ such that $X=\operatorname{CGr}\left(2, V_{5}\right) \cap Q$. Let $I_{0} \subset W$ be a general $Q$-isotropic 6 -subspace, so that $S_{0}:=\mathbf{P}\left(I_{0}\right) \cap X$ is a smooth quintic del Pezzo surface. Consider the linear projection

$$
\pi_{S_{0}}: X \rightarrow \mathbf{P}^{n-2}
$$

from the linear span $\mathbf{P}\left(I_{0}\right)$ of $S_{0}$, where $n=\operatorname{dim}(X)$. A general fiber is also a quintic del Pezzo surface. Indeed, the intersection of a general $\mathbf{P}^{6}$ containing $\mathbf{P}\left(I_{0}\right)=\mathbf{P}^{5}$ with $X$ is a 2-dimensional GM intersection containing the quintic del Pezzo surface $S_{0}$. It is the union of $S_{0}$ and a residual quintic del Pezzo surface $S$ (more precisely, the intersection of $Q$ with this $\mathbf{P}^{6}$ is the union of $\mathbf{P}\left(I_{0}\right)$ and a residual space $\mathbf{P}(I)$, where $I$ is another $Q$-isotropic 6-subspace, and $S=\mathbf{P}(I) \cap X)$.

The argument of the proof of Lemma 4.1 shows that for a general choice of $S_{0}$, the general fiber of $\pi_{S_{0}}$ is smooth. Hence the field of rational functions on $X$ is the field of rational functions on a smooth quintic del Pezzo surface defined over the field of rational functions on $\mathbf{P}^{n-2}$. But a smooth quintic del Pezzo surface is rational over any field by a theorem of Enriques (see [She92]).

Remark 4.3. Let $X$ be a smooth complex GM fourfold such that $Y_{A(X)}^{3} \backslash \mathbf{P}\left(V_{5}(X)\right) \neq \varnothing$. The same argument shows that $X$ is rational. More generally, using Theorem 4.15, one can show that $X$ is rational as soon as $Y_{A(X)}^{3} \neq \varnothing$ (see [KP16, Lemma 6.7] for more details). 


\section{Gushel-Mukai VARIETies}

\subsection{The first quadric fibration}

Let $X$ be a smooth GM variety of dimension $n \geqslant 3$, with extended Lagrangian data $\left(V_{6}, V_{5}, A, A_{1}\right)$ and GM data $\left(W, V_{6}, V_{5}, L, \mu, \mathbf{q}, \varepsilon\right)$. By Theorem 3.16, the space $A$ contains no decomposable vectors. Consider the Gushel bundle $\mathscr{U}_{X}$ and its projectivization $\mathbf{P}_{X}\left(\mathscr{U}_{X}\right)$. The canonical embedding $\mathscr{U}_{X} \hookrightarrow V_{5} \otimes \mathscr{O}_{X}$ induces a regular map

$$
\rho_{1}: \mathbf{P}_{X}\left(\mathscr{U}_{X}\right) \rightarrow \mathbf{P}\left(V_{5}\right) .
$$

In Proposition 4.5, we prove that $\rho_{1}$ is a quadric fibration and identify its discriminant locus.

Let $W_{0}^{\perp}=A \cap \bigwedge^{3} V_{5}$ be the even part of the space of linear equations of $X$. We consider $W_{0}^{\perp}$ as a subspace of the space $\bigwedge^{2} V_{5}^{\vee}$ of skew forms on $V_{5}$. Let

$$
\Sigma_{1}(X) \subset \mathbf{P}\left(V_{5}\right)
$$

be the union of the kernels of all non-zero skew forms in the space $W_{0}^{\perp}$. It can be described in terms of the incidence correspondence $\widehat{Y}_{A}$ and its two projections $p: \widehat{Y}_{A} \rightarrow Y_{A} \subset \mathbf{P}\left(V_{6}\right)$ and $q: \widehat{Y}_{A} \rightarrow Y_{A^{\perp}} \subset \mathbf{P}\left(V_{6}^{\vee}\right)($ see $(\mathrm{B} .5))$.

Lemma 4.4. We have $\Sigma_{1}(X)=p\left(q^{-1}\left(\left[V_{5}\right]\right)\right)$. In particular, $\Sigma_{1}(X) \subset Y_{A} \cap \mathbf{P}\left(V_{5}\right)$. Moreover, $\operatorname{dim}\left(\Sigma_{1}(X)\right) \leqslant 1$ if $n=4$, and $\operatorname{dim}\left(\Sigma_{1}(X)\right) \leqslant 2$ if $n=3$.

Proof. The lemma follows immediately from Remark B.4.

We now prove an analogue of Proposition 3.18.

Proposition 4.5. Let $X$ be a smooth $G M$ variety of dimension $n \geqslant 3$.

Over $\mathbf{P}\left(V_{5}\right) \backslash \Sigma_{1}(X)$, the map $\rho_{1}: \mathbf{P}_{X}\left(\mathscr{U}_{X}\right) \rightarrow \mathbf{P}\left(V_{5}\right)$ is a relative quadratic hypersurface in a $\mathbf{P}^{n-2}$-fibration, whose fiber over a point $v$ has corank $k$ if and only if $v \in Y_{A}^{k} \cap \mathbf{P}\left(V_{5}\right)$. In particular, in $\mathbf{P}\left(V_{5}\right) \backslash \Sigma_{1}(X)$, its discriminant locus is $Y_{A} \cap\left(\mathbf{P}\left(V_{5}\right) \backslash \Sigma_{1}(X)\right)$.

Over $\Sigma_{1}(X)$, the map $\rho_{1}$ is a relative quadratic hypersurface in a $\mathbf{P}^{n-1}$-fibration, whose fiber over a point $v$ has corank $k$ if and only if $v \in Y_{A}^{k+1}$.

Proof. Choose an arbitrary $v_{0} \in V_{6} \backslash V_{5}$, and let $v \in V_{5}$. The fiber $\rho_{1}^{-1}(v)$ is the set of $V_{2} \subset V_{5}$ containing $v$ which correspond to points of $X$. This is the intersection $Q^{\prime}(v)$ of the quadric $Q\left(v_{0}\right) \subset \mathbf{P}(W)$ defining $X$ with the subspace $\mathbf{P}\left(\left(\left(v \wedge V_{5}\right) \oplus \mathbf{k}\right) \cap W\right) \subset \mathbf{P}(W)$.

On the other hand, by the argument of Theorem 3.6, the quadric $Q\left(v_{0}\right)$ is the quadric corresponding to the Lagrangian subspace $\hat{A}=A \oplus A_{1} \subset \mathbb{V}$ with respect to the Lagrangian decomposition $\mathbb{V}=\mathbb{L}_{1} \oplus \mathbb{L}_{2}$ of (3.4), with $\mathbb{L}_{1}=\bigwedge^{3} V_{5} \oplus L$ and $\mathbb{L}_{2}=\left(v_{0} \wedge \bigwedge^{2} V_{5}\right) \oplus \mathbf{k}$, under the Lagrangian-quadratic correspondence of Appendix C (it is the quadric $Q_{2}^{\hat{A}}$ of Proposition C.1). Therefore, the intersection

$$
\left.Q^{\prime}(v)=Q\left(v_{0}\right) \cap \mathbf{P}\left(\left(v \wedge V_{5}\right) \oplus \mathbf{k}\right) \cap W\right)
$$

can be described by the isotropic reduction of Corollary C.4.

For this, we set $\overline{\mathbb{L}}_{2}:=\left(v_{0} \wedge v \wedge V_{5}\right) \oplus \mathbf{k} \subset \mathbb{L}_{2}$, and we consider the isotropic subspace

$$
\mathbb{I}_{v}:=\mathbb{L}_{1} \cap \overline{\mathbb{L}}_{2}^{\perp}=v \wedge \wedge^{2} V_{5} \subset \mathbb{L}_{1} .
$$

According to Corollary C.4, the codimension in $\mathbf{P}\left(\overline{\mathbb{L}}_{2}\right)$ of the span of the quadric $Q^{\prime}(v)$ is equal to $\operatorname{dim}\left(\left(\hat{A} \cap \mathbb{L}_{1}\right) /\left(\hat{A} \cap \mathbb{I}_{v}\right)\right)$, and its kernel is equal to $\left(\hat{A} \cap\left(\mathbb{I}_{v} \oplus \overline{\mathbb{L}}_{2}\right)\right) /\left(\hat{A} \cap \mathbb{I}_{v}\right)$, so it remains to describe the intersections of $\hat{A}$ with $\mathbb{I}_{v}, \mathbb{L}_{1}$, and $\mathbb{I}_{v} \oplus \overline{\mathbb{L}}_{2}$. 


\section{O. Debarre and A. Kuznetsov}

First, we have $\hat{A} \cap \mathbb{I}_{v}=A \cap\left(v \wedge \wedge^{2} V_{5}\right) \subset A \cap \bigwedge^{3} V_{5}=W_{0}^{\perp}$, where $W_{0}^{\perp}$ is the even part of the space of linear equations of $X$. A skew form on $V_{5}$, considered as an element of $\wedge^{3} V_{5}$, belongs to $v \wedge \wedge^{2} V_{5}$ if and only if $v$ is in its kernel. This means that $\hat{A} \cap \mathbb{I}_{v}$ is non-zero only for $v \in \Sigma_{1}(X)$, and the intersection $\hat{A} \cap \mathbb{I}_{v}$ is then 1-dimensional, since otherwise the space $A \cap\left(v \wedge \wedge^{2} V_{5}\right)$ would contain decomposable vectors. Therefore,

$$
\operatorname{dim}\left(\hat{A} \cap \mathbb{I}_{v}\right)= \begin{cases}1 & \text { if } v \in \Sigma_{1}(X), \\ 0 & \text { if } v \notin \Sigma_{1}(X) .\end{cases}
$$

Since the space $\hat{A} \cap \mathbb{L}_{1}=W^{\perp}$ has dimension $6-n$, the codimension of the span of $Q^{\prime}(v)$ in $\mathbf{P}\left(\overline{\mathbb{L}}_{2}\right)$ is $5-n$ for $v \in \Sigma_{1}(X)$ and $6-n$ for $v \notin \Sigma_{1}(X)$. Since the dimension of $\mathbf{P}\left(\overline{\mathbb{L}}_{2}\right)$ is 4 , it follows that $Q^{\prime}(v)$ is a quadratic hypersurface in $\mathbf{P}^{n-1}$ or $\mathbf{P}^{n-2}$, depending on whether $v$ is in $\Sigma_{1}(X)$ or not. Finally, we have

$$
\mathbb{I}_{v} \oplus \overline{\mathbb{L}}_{2}=\left(\left(v \wedge \wedge^{2} V_{5}\right) \oplus\left(v_{0} \wedge v \wedge V_{5}\right)\right) \oplus \mathbf{k}=v \wedge \wedge^{2} V_{6} \oplus \mathbf{k}
$$

Therefore, the dimension of the intersection $\hat{A} \cap\left(\mathbb{I}_{v} \oplus \overline{\mathbb{L}}_{2}\right)=A \cap\left(v \wedge \wedge^{2} V_{6}\right)$ is detected by the position of $v \in \mathbf{P}\left(V_{6}\right)$ with respect to the EPW stratification defined by $A$ on $\mathbf{P}\left(V_{6}\right)$. Taking into account (4.1), we obtain that for $v \in Y_{A}^{k} \backslash \Sigma_{1}(X)$, the corank of $Q^{\prime}(v)$ is $k$, and for $v \in Y_{A}^{k} \cap \Sigma_{1}(X)$, the corank of $Q^{\prime}(v)$ is $k-1$ (this gives another proof of the inclusion $\Sigma_{1}(X) \subset Y_{A}$ ).

\subsection{The birationality of period partners of dimension 3}

Let $X_{1}$ and $X_{2}$ be smooth complex GM threefolds. Assume that they are period partners, so that they are constructed from Lagrangian data sets with the same $V_{6}$ and $A$, but possibly different Plücker hyperplanes $V_{5}^{1} \subset V_{6}$ and $V_{5}^{2} \subset V_{6}$ and possibly different Lagrangian subspaces $A_{1}$ and $A_{1}^{\prime}$. The aim of this section is to prove that the varieties $X_{1}$ and $X_{2}$ are birationally isomorphic.

Consider the diagrams
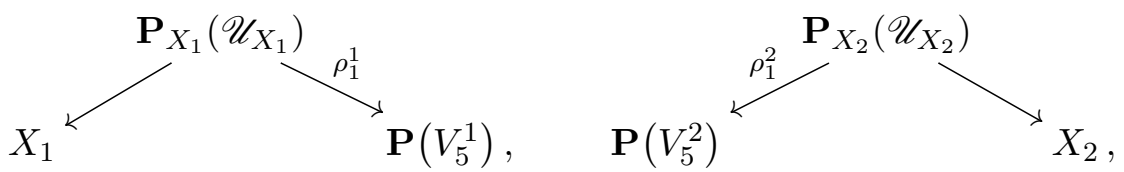

where $\rho_{1}^{1}$ and $\rho_{1}^{2}$ are the first quadratic fibrations associated with the threefolds $X_{1}$ and $X_{2}$ respectively. Denote by $\Sigma_{1}\left(X_{1}\right) \subset Y_{A} \cap \mathbf{P}\left(V_{5}^{1}\right)$ and $\Sigma_{1}\left(X_{2}\right) \subset Y_{A} \cap \mathbf{P}\left(V_{5}^{2}\right)$ the associated subsets of the previous section. Assume moreover $V_{5}^{1} \neq V_{5}^{2}$, set $V_{4}:=V_{5}^{1} \cap V_{5}^{2} \subset V_{6}$, and restrict everything to the common base $\mathbf{P}\left(V_{4}\right)$. We get a diagram

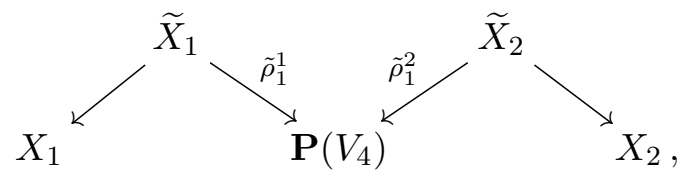

where $\widetilde{X}_{i}=\mathbf{P}_{X_{i}}\left(\mathscr{U}_{X_{i}}\right) \times_{\mathbf{P}\left(V_{5}^{1}\right)} \mathbf{P}\left(V_{4}\right)$ and $\tilde{\rho}_{1}^{i}$ is the restriction of $\rho_{1}^{i}$ to $\widetilde{X}_{i}$.

Lemma 4.6. For each $i \in\{1,2\}$, the map $\widetilde{X}_{i} \rightarrow X_{i}$ is the blow-up of $X_{i} \cap \mathrm{CGr}\left(2, V_{4}\right)$. In particular, $\widetilde{X}_{i}$ is irreducible, generically reduced, and birational to $X_{i}$.

Proof. By definition, $\widetilde{X}_{1}=\mathbf{P}_{X_{1}}\left(\mathscr{U}_{X_{1}}\right) \times_{\mathbf{P}\left(V_{5}\right)} \mathbf{P}\left(V_{4}\right)$ is a divisor in a smooth 4-dimensional variety $\mathbf{P}_{X_{1}}\left(\mathscr{U}_{X_{1}}\right)$; hence each irreducible component of $\widetilde{X}_{1}$ is at least 3-dimensional. The fiber of 


\section{Gushel-Mukai VARIETIES}

$\widetilde{X}_{1} \rightarrow X_{1}$ over a point $w \in X_{1}$ is a linear section of the fiber of $\mathbf{P}_{X_{1}}\left(\mathscr{U}_{X_{1}}\right) \rightarrow X_{1}$, so it is a point if $\mathscr{U}_{X_{1}, w} \not \subset V_{4}$ and the line $\mathbf{P}\left(\mathscr{U}_{X_{1}, w}\right)$ if $\mathscr{U}_{X_{1}, w} \subset V_{4}$. Therefore, the locus of non-trivial fibers is $X_{1} \cap \operatorname{CGr}\left(2, V_{4}\right)$.

To show that the map $\widetilde{X}_{1} \rightarrow X_{1}$ is the blow-up, it is enough by [Kuz16, Lemma 2.1] to check that the intersection $X_{1} \cap \operatorname{CGr}\left(2, V_{4}\right)$ has dimension at most 1 . Since $X_{1}$ is cut out in $\operatorname{CGr}\left(2, V_{5}\right)$ by three hyperplanes and a quadric, the intersection $X_{1} \cap \operatorname{CGr}\left(2, V_{4}\right)$ is also cut out in $\operatorname{CGr}\left(2, V_{4}\right)$ by three hyperplanes and a quadric; hence its dimension is at least 1 and its degree is at most 4 . But $X_{1}$ is a smooth threefold with $\operatorname{Pic}\left(X_{1}\right)=\mathbf{Z} H$, so by the Lefschetz theorem, it contains no surfaces of degree less than $H^{3}=10$; hence we have $\operatorname{dim}\left(X_{1} \cap \operatorname{CGr}\left(2, V_{4}\right)\right)=1$.

The same argument works for $\widetilde{X}_{2}$ as well.

If $X_{1}$ and $X_{2}$ are non-isomorphic period partners, that is if $A\left(X_{1}\right)=A\left(X_{2}\right)$, then by Theorem 3.25 , we have $V_{5}\left(X_{1}\right) \neq V_{5}\left(X_{2}\right)$.

Theorem 4.7. Let $X_{1}$ and $X_{2}$ be smooth complex GM threefolds which are period partners, that is, such that $A\left(X_{1}\right)=A\left(X_{2}\right)$ and $V_{5}\left(X_{1}\right) \neq V_{5}\left(X_{2}\right)$. Let $V_{4}=V_{5}\left(X_{1}\right) \cap V_{5}\left(X_{2}\right) \subset V_{6}$. Over an open subset $U \subset \mathbf{P}\left(V_{4}\right)$, there is an isomorphism $\left(\tilde{\rho}_{1}^{1}\right)^{-1}(U) \simeq\left(\tilde{\rho}_{1}^{2}\right)^{-1}(U)$. In particular, $\widetilde{X}_{1}$ is birationally isomorphic to $\widetilde{X}_{2}$ over $\mathbf{P}\left(V_{4}\right)$, and $X_{1}$ is birationally isomorphic to $X_{2}$.

Proof. By Proposition 4.5, the morphism $\rho_{1}^{1}: \mathbf{P}_{X_{1}}\left(\mathscr{U}_{X_{1}}\right) \rightarrow \mathbf{P}\left(V_{5}\left(X_{1}\right)\right)$ is, outside the locus $\Sigma_{1}\left(X_{1}\right) \subset \mathbf{P}\left(V_{5}\right)$ (which has dimension at most 2 by Lemma 4.4), a flat double cover branched along the sextic $Y_{A} \cap \mathbf{P}\left(V_{5}\left(X_{1}\right)\right)$. The morphism $\tilde{\rho}_{1}^{1}: \widetilde{X}_{1} \rightarrow \mathbf{P}\left(V_{4}\right)$ is obtained from $\rho_{1}^{1}$ by a base change.

If $\widetilde{X}_{1} \stackrel{g_{1}}{\longrightarrow} \bar{X}_{1} \stackrel{h_{1}}{\longrightarrow} \mathbf{P}\left(V_{4}\right)$ is the Stein factorization of $\tilde{\rho}_{1}^{1}$, then $g_{1}$ is birational and $\bar{X}_{1}$ is isomorphic to the restriction of $\operatorname{Spec}\left(\rho_{1 *}^{1} \mathscr{O}_{\mathbf{P}_{X_{1}}\left(\mathscr{U}_{X_{1}}\right)}\right)$ to $\mathbf{P}\left(V_{4}\right)$; hence $h_{1}$ is the double cover of $\mathbf{P}\left(V_{4}\right)$ branched along $Y_{A} \cap \mathbf{P}\left(V_{4}\right)$ (note that $Y_{A} \cap \mathbf{P}\left(V_{4}\right) \neq \mathbf{P}\left(V_{4}\right)$ since $\widetilde{X}_{1}$, hence also $\bar{X}_{1}$, is generically reduced).

The same argument shows that in the Stein factorization $\tilde{\rho}_{1}^{2}: \tilde{X}_{2} \stackrel{g_{2}}{\longrightarrow} \bar{X}_{2} \stackrel{h_{2}}{\longrightarrow} \mathbf{P}\left(V_{4}\right)$, the map $g_{2}$ is also birational and $h_{2}$ is again the double covering branched along the same sextic $Y_{A} \cap \mathbf{P}\left(V_{4}\right)$.

Since the branch divisors coincide, $\bar{X}_{1}$ and $\bar{X}_{2}$ are isomorphic, and $\widetilde{X}_{1}$ and $\widetilde{X}_{2}$ are birationally isomorphic. By Lemma 4.6, the threefolds $X_{1}$ and $X_{2}$ are birationally isomorphic as well.

Remark 4.8. One can explicitly decompose the birational transformation $X_{1} \stackrel{\sim}{\sim} \rightarrow X_{2}$ into several steps. The first half can be described as follows:

- The map $\tilde{X}_{1} \rightarrow X_{1}$ is the blow-up of the union $X_{1} \cap \operatorname{CGr}\left(2, V_{4}\right)=c_{1} \cup c_{2}$ of two conics intersecting in two points.

- The map $\widetilde{X}_{1} \rightarrow \bar{X}_{1}$ contracts all lines intersecting $c_{1} \cup c_{2}$ and all conics intersecting $c_{1} \cup c_{2}$ twice (these conics are $\sigma$-conics and correspond to points of $\Sigma_{1} \cap \mathbf{P}\left(V_{4}\right)$ ).

The second part can be described similarly. This transformation is very similar to the conic transformation used in [DIM12, Theorem 7.4] to prove Theorem 4.7 when $X_{1}$ is a general GM threefold.

One can use the same idea to prove the birationality of period partners in any dimension $n \geqslant 3$. It requires, however, a long and cumbersome analysis, so we decided to omit it. Instead, we will use birationalities for dual GM varieties (proved later in this section) to establish the 


\section{O. Debarre And A. KuZnetsov}

result in dimension 4 (Theorem 4.15). Since in dimensions $n \geqslant 5$, all smooth GM varieties are rational, this shows that the result holds in all dimensions $n \geqslant 3$.

\subsection{The second quadric fibration}

Let $X$ be a smooth GM variety of dimension $n \geqslant 3$. Instead of the Gushel bundle $\mathscr{U}_{X}$, we now consider the quotient $\left(V_{5} \otimes \mathscr{O}_{X}\right) / \mathscr{U}_{X}$, which we denote simply by $V_{5} / \mathscr{U}_{X}$, its projectivization $\mathbf{P}_{X}\left(V_{5} / \mathscr{U}_{X}\right)$, and the map $\rho_{2}: \mathbf{P}_{X}\left(V_{5} / \mathscr{U}_{X}\right) \rightarrow \operatorname{Gr}\left(3, V_{5}\right)$. In Proposition 4.10 , we prove that $\rho_{2}$ is a quadric fibration and identify its discriminant locus.

Let, as before, $W_{0}^{\perp}=A \cap \bigwedge^{3} V_{5}$ be the even part of the space of linear equations of $X$. Considering its elements as skew forms on $V_{5}$, we denote by

$$
\Sigma_{2}^{\geqslant k}(X) \subset \operatorname{Gr}\left(3, V_{5}\right)
$$

the set of all 3-dimensional subspaces $V_{3}$ in $V_{5}$ which are isotropic for all elements of some $k$-dimensional space of these skew forms. As usual, set $\Sigma_{2}(X):=\Sigma_{2}^{\geqslant 1}(X)$.

Finally, we will use the EPW sequence

$$
Z_{A}^{\geqslant k}=\left\{V_{3} \in \operatorname{Gr}\left(3, V_{6}\right) \mid \operatorname{dim}\left(A \cap\left(V_{6} \wedge \wedge^{2} V_{3}\right)\right) \geqslant k\right\}
$$

defined in (B.10) and set $Z_{A}:=Z_{A}^{\geqslant 1}$. This sequence plays the same role for the second quadratic fibration as the EPW sequence of $\mathbf{P}\left(V_{6}\right)$ plays for the first.

In the next lemma, we consider 1-dimensional subspaces $V_{1} \subset V_{5}$ and identify the Grassmannian $\operatorname{Gr}\left(2, V_{5} / V_{1}\right)$ with the subscheme of $\operatorname{Gr}\left(3, V_{5}\right)$ parameterizing all 3-dimensional subspaces containing $V_{1}$.

Lemma 4.9. For any smooth $G M$ variety $X$ of dimension $n \geqslant 3$, we have $\Sigma_{2}(X) \subset Z_{A} \cap \operatorname{Gr}\left(3, V_{5}\right)$.

Assume, moreover, that either $n=3$ and $X$ is ordinary, or $n \geqslant 4$. If $V_{1} \notin \Sigma_{1}(X)$, we have $\operatorname{dim}\left(\Sigma_{2}(X) \cap \operatorname{Gr}\left(2, V_{5} / V_{1}\right)\right) \leqslant 2$.

Proof. Let $V_{3} \in \Sigma_{2}(X)$; that is, the subspace $V_{3}$ is isotropic for some non-zero $\omega \in A \cap \bigwedge^{3} V_{5}$, considered as a skew form on $V_{5}$. Then $\omega \in \bigwedge^{2} V_{3} \wedge V_{5}$. It follows that $V_{3} \in Z_{A}$, which proves the first assertion of the lemma.

Let $\omega \in A \cap \bigwedge^{3} V_{5}$ be non-zero. Since $X$ is smooth of dimension at least 3 , the Lagrangian $A$ contains no decomposable vectors; hence $\omega$, viewed as a skew form on $V_{5}$, has rank 4 and its kernel is a 1-dimensional subspace $K_{1}(\omega) \subset V_{5}$. Any 3-dimensional subspace $V_{3}$ of $V_{5}$ which is isotropic for $\omega$ contains $K_{1}(\omega)$; hence the set of such subspaces can be identified with the set of 2-dimensional subspaces in $V_{5} / K_{1}(\omega)$ which are isotropic for the non-degenerate skew form induced by $\omega$, that is, with a 3 -dimensional quadric $\operatorname{LGr}(2,4)$.

It remains to describe which of these subspaces $V_{3}$ contain $V_{1}$. Since $V_{1}$ is not in $\Sigma_{1}(X)$, it projects onto a non-zero subspace $V_{1}^{\prime} \subset V_{5} / K_{1}(\omega)$. The subset of $\operatorname{LGr}(2,4)$ consisting of subspaces containing $V_{1}^{\prime}$ is $\mathbf{P}\left(V_{1}^{\perp} / V_{1}^{\prime}\right)=\mathbf{P}^{1}$. It follows that $\Sigma_{2}(X) \cap \operatorname{Gr}\left(2, V_{5} / V_{1}\right)$ is dominated by a $\mathbf{P}^{1}$. fibration over $\mathbf{P}\left(A \cap \bigwedge^{3} V_{5}\right)$. Since $\operatorname{dim}\left(A \cap \bigwedge^{3} V_{5}\right) \leqslant 2$ for GM fourfolds and ordinary GM threefolds (see (3.9)), the claim follows.

Proposition 4.10. Let $X$ be a smooth $G M$ variety of dimension $n \geqslant 3$.

Over $\operatorname{Gr}\left(3, V_{5}\right) \backslash \Sigma_{2}(X)$, the map $\rho_{2}: \mathbf{P}_{X}\left(V_{5} / \mathscr{U}_{X}\right) \rightarrow \operatorname{Gr}\left(3, V_{5}\right)$ is a relative quadratic hypersurface in a $\mathbf{P}^{n-3}$-fibration, whose fiber over a point $U_{3}$ has corank $k$ if and only if $U_{3} \in Z_{A}^{k}$. In particular, in $\operatorname{Gr}\left(3, V_{5}\right) \backslash \Sigma_{2}(X)$, its discriminant locus is $Z_{A} \cap\left(\operatorname{Gr}\left(3, V_{5}\right) \backslash \Sigma_{2}(X)\right)$.

Over $\Sigma_{2}^{l}(X)$, the map $\rho_{2}: \mathbf{P}_{X}\left(V_{5} / \mathscr{U}_{X}\right) \rightarrow \mathrm{Gr}\left(3, V_{5}\right)$ is a relative quadratic hypersurface in a $\mathbf{P}^{n+l-3}$-fibration, whose fiber over a point $U_{3}$ has corank $k$ if and only if $U_{3} \in Z_{A}^{k+l}$. 


\section{Gushel-Mukai VARIETIES}

Proof. We follow the proof of Proposition 4.5 and use the notation introduced therein. Choose $v_{0} \in V_{6} \backslash V_{5}$, and let $V_{3} \subset V_{5}$. The fiber $\rho_{2}^{-1}\left(V_{3}\right)$ is the set of $V_{2} \subset V_{3}$ which correspond to points of $X$. This is the intersection

$$
Q^{\prime}\left(V_{3}\right):=Q\left(v_{0}\right) \cap \mathbf{P}\left(\left(\bigwedge^{2} V_{3} \oplus \mathbf{k}\right) \cap W\right),
$$

so it can be described by the isotropic reduction of Corollary C.4.

For this, we let $\overline{\mathbb{L}}_{2}:=\left(v_{0} \wedge \wedge^{2} V_{3}\right) \oplus \mathbf{k} \subset \mathbb{L}_{2}$, and we consider the isotropic subspace

$$
\mathbb{I}_{V_{3}}:=\mathbb{L}_{1} \cap \overline{\mathbb{L}}_{2}^{\perp}=\left(\bigwedge^{3} V_{5} \oplus L\right) \cap\left(\bigwedge^{2} V_{3} \oplus \mathbf{k}\right)^{\perp}=V_{5} \wedge \bigwedge^{2} V_{3} .
$$

According to Corollary C.4, the codimension in $\mathbf{P}\left(\overline{\mathbb{L}}_{2}\right)$ of the span of the quadric $Q^{\prime}\left(V_{3}\right)$ is equal to $\operatorname{dim}\left(\left(\hat{A} \cap \mathbb{L}_{1}\right) /\left(\hat{A} \cap \mathbb{I}_{V_{3}}\right)\right)$ and its kernel is $\left(\hat{A} \cap\left(\mathbb{I}_{V_{3}} \oplus \overline{\mathbb{L}}_{2}\right)\right) /\left(\hat{A} \cap \mathbb{I}_{V_{3}}\right)$. It remains to describe the intersections of $\hat{A}$ with $\mathbb{I}_{V_{3}}, \mathbb{L}_{1}$, and $\mathbb{I}_{V_{3}} \oplus \overline{\mathbb{L}}_{2}$.

We have $\hat{A} \cap \mathbb{I}_{V_{3}}=A \cap\left(V_{5} \wedge \wedge^{2} V_{3}\right) \subset A \cap \wedge^{3} V_{5}=W_{0}^{\perp}$. A skew form on $V_{5}$, considered as an element of $\wedge^{3} V_{5}$, is contained in the subspace $V_{5} \wedge \wedge^{2} V_{3}$ if and only if $V_{3}$ is isotropic for it. This means that for $V_{3} \in \Sigma_{2}^{l}(X)$, we have

$$
\operatorname{dim}\left(\hat{A} \cap \mathbb{I}_{V_{3}}\right)=l .
$$

Furthermore, the space $\hat{A} \cap \mathbb{L}_{1}=W^{\perp}$ has dimension $6-n$; hence the codimension of the span of $Q^{\prime}\left(V_{3}\right)$ in $\mathbf{P}\left(\overline{\mathbb{L}}_{2}\right)$ is $6-n-l$. Since the dimension of $\mathbf{P}\left(\overline{\mathbb{L}}_{2}\right)$ is 3 , it follows that $Q^{\prime}\left(V_{3}\right)$ is a quadratic hypersurface in $\mathbf{P}^{n+l-3}$. Finally, we have

$$
\mathbb{I}_{V_{3}} \oplus \overline{\mathbb{L}}_{2}=\left(V_{5} \wedge \wedge^{2} V_{3}\right) \oplus\left(v_{0} \wedge \wedge^{2} V_{3}\right) \oplus \mathbf{k}=V_{6} \wedge \bigwedge^{2} V_{3} \oplus \mathbf{k}
$$

Therefore, the dimension of $\hat{A} \cap\left(\mathbb{I}_{V_{3}} \oplus \overline{\mathbb{L}}_{2}\right)=A \cap\left(V_{6} \wedge \wedge^{2} V_{3}\right)$ is detected by the position of the space $V_{3} \in \operatorname{Gr}\left(3, V_{5}\right)$ with respect to the stratification $Z_{A}^{\bullet}$ of $\operatorname{Gr}\left(3, V_{6}\right)$ defined in (B.10). Taking (4.3) into account, we deduce that for $V_{3} \in Z_{A}^{k} \cap \Sigma_{2}^{l}(X)$, the corank of $Q^{\prime}(v)$ is $k-l$.

\subsection{The birationality of dual varieties of dimension 4}

Let $X$ and $X^{\prime}$ be smooth dual GM varieties of the same dimension $n \geqslant 3$. This means that they are constructed from dual Lagrangian data sets $\left(V_{6}, V_{5}, A, A_{1}\right)$ and $\left(V_{6}^{\vee}, V_{5}^{\prime}, A^{\perp}, A_{1}^{\prime}\right)$. Set $V_{1}:=V_{5}^{\prime \perp} \subset V_{6}$ and assume additionally

$$
V_{1} \subset V_{5}
$$

(we will see later that the general case reduces to this one).

Remark 4.11. The condition (4.4) is symmetric with respect to $X$ and $X^{\prime}$. Indeed, it can be reformulated as the degeneracy of the restriction of the natural pairing $V_{6} \otimes V_{6}^{\vee} \rightarrow \mathbf{k}$ to the subspace $V_{5} \otimes V_{5}^{\prime} \subset V_{6} \otimes V_{6}^{\vee}$.

The construction of birationalities is very similar to the one used in Section 4.3. Assume $n=3$ or $n=4$, and consider the diagrams

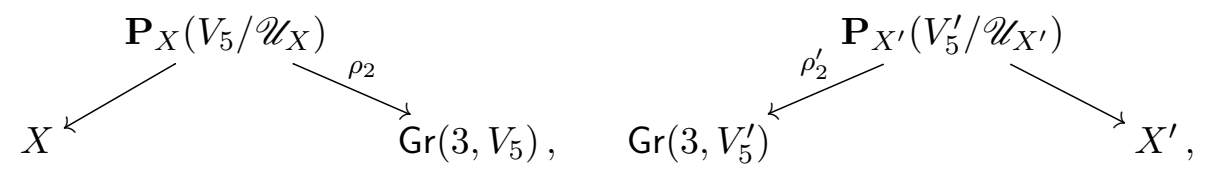

where $\rho_{2}$ and $\rho_{2}^{\prime}$ are the second quadric fibrations defined in Section 4.4, together with the subsets $\Sigma_{2}(X) \subset Z_{A} \cap \operatorname{Gr}\left(3, V_{5}\right)$ and $\Sigma_{2}\left(X^{\prime}\right) \subset Z_{A} \cap \operatorname{Gr}\left(3, V_{5}^{\prime}\right)$. We have $\operatorname{Gr}\left(3, V_{5}\right) \subset \operatorname{Gr}\left(3, V_{6}\right)$ and 


\section{O. Debarre and A. Kuznetsov}

$\operatorname{Gr}\left(3, V_{5}^{\prime}\right) \subset \operatorname{Gr}\left(3, V_{6}^{\vee}\right)=\operatorname{Gr}\left(3, V_{6}\right)$, and, since (4.4) is satisfied, their intersection is

$$
\operatorname{Gr}\left(2, V_{5} / V_{1}\right) \simeq \operatorname{Gr}(2,4)
$$

(it is empty if (4.4) does not hold). We restrict everything to the common base $\operatorname{Gr}\left(2, V_{5} / V_{1}\right)$ and get a diagram

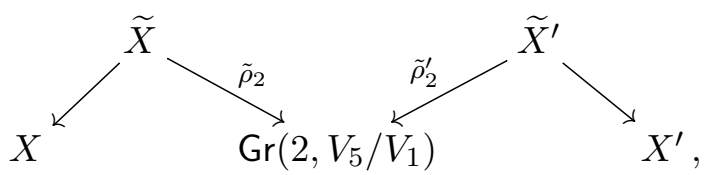

where $\widetilde{X}=\mathbf{P}_{X}\left(V_{5} / \mathscr{U}_{X}\right) \times{ }_{\operatorname{Gr}\left(3, V_{5}\right)} \operatorname{Gr}\left(2, V_{5} / V_{1}\right)$ and $\tilde{\rho}_{2}$ is the restriction of $\rho_{2}$ to $\tilde{X}$, and analogously for $\tilde{X}^{\prime}$ and $\tilde{\rho}_{2}^{\prime}$.

LEMMA 4.12. If

$$
V_{1} \notin \Sigma_{1}(X) \cup Y_{A(X)}^{\geqslant n-1}
$$

the map $\widetilde{X} \rightarrow X$ is the blow-up of $X \cap \operatorname{CGr}\left(1, V_{5} / V_{1}\right)$. In particular, $\widetilde{X}$ is irreducible, generically reduced, and birational to $X$. Analogous claims hold for $\widetilde{X}^{\prime}$.

Proof. The proof is analogous to that of Lemma 4.6. The fiber of the map $\widetilde{X} \rightarrow X$ over a point $w \in X$ is a point if $V_{1} \not \subset \mathscr{U}_{w}$ and $\mathbf{P}\left(V / \mathscr{U}_{w}\right) \simeq \mathbf{P}^{2}$ otherwise. The locus of non-trivial fibers is therefore $X \cap \operatorname{CGr}\left(1, V_{5} / V_{1}\right)$. Since $X \subset \operatorname{CGr}\left(2, V_{5}\right)$ is an intersection of $6-n$ hyperplanes and a quadric, $X \cap \operatorname{CGr}\left(1, V_{5} / V_{1}\right)$ is the intersection of $6-n$ hyperplanes and a quadric in $\operatorname{CGr}\left(1, V_{5} / V_{1}\right)=\mathbf{P}^{4}$; hence its expected dimension is $n-3$.

The dimension can jump if

- either one of the hyperplanes contains $\operatorname{CGr}\left(1, V_{5} / V_{1}\right)$, that is, $V_{1} \in \Sigma_{1}(X)$;

- or the quadric contains the intersection $\mathbf{P}(W) \cap \operatorname{CGr}\left(1, V_{5} / V_{1}\right)=\mathbf{P}^{n-2}$; this is equivalent to $V_{1} \in Y_{A(X)}^{\geqslant n-1}$ by Proposition 4.5.

So, if (4.6) is satisfied, the dimension does not jump and $\operatorname{dim}\left(X \cap \operatorname{CGr}\left(1, V_{5} / V_{1}\right)\right) \leqslant n-3$. The exceptional set of $\widetilde{X} \rightarrow X$ has dimension at most $n-1$ and thus cannot be an irreducible component of $\widetilde{X}$. It follows that $\widetilde{X}$ is integral and the map $\widetilde{X} \rightarrow X$ is the blow-up of $X \cap$ $\operatorname{CGr}\left(1, V_{5} / V_{1}\right)$. The same argument works for $\tilde{X}^{\prime}$.

Proposition 4.13. Let $X$ and $X^{\prime}$ be smooth dual complex GM fourfolds satisfying (4.4) and such that (4.6) holds for both $X$ and $X^{\prime}$. Over a dense open subset $U \subset \operatorname{Gr}\left(2, V_{5} / V_{1}\right)$, there is an isomorphism $\tilde{\rho}_{2}^{-1}(U) \simeq \tilde{\rho}_{2}^{\prime-1}(U)$. In particular, $\widetilde{X}$ is birationally isomorphic to $\widetilde{X}^{\prime}$ over $\operatorname{Gr}\left(2, V_{5} / V_{1}\right)$, and $X$ is birationally isomorphic to $X^{\prime}$.

Proof. By Proposition 4.10, the morphism $\rho_{2}: \mathbf{P}_{X}\left(V_{5} / \mathscr{U}_{X}\right) \rightarrow \operatorname{Gr}\left(3, V_{5}\right)$ is, outside of the locus $\Sigma_{2}(X) \subset \operatorname{Gr}\left(3, V_{5}\right)$, a flat double cover branched along the quartic $Z_{A} \cap \operatorname{Gr}\left(3, V_{5}\right)$. The morphism $\tilde{\rho}_{2}: \widetilde{X} \rightarrow \operatorname{Gr}\left(2, V_{5} / V_{1}\right)$ is obtained from $\rho_{2}$ by a base change. Outside of $\Sigma_{2}(X) \cap \operatorname{Gr}\left(2, V_{5} / V_{1}\right)$ (which has dimension at most 2 by Lemma 4.9), $\tilde{\rho}_{2}$ is therefore a double covering branched along $Z_{A} \cap \operatorname{Gr}\left(2, V_{5} / V_{1}\right)$.

Let $\tilde{X} \stackrel{g}{\longrightarrow} \bar{X} \stackrel{h}{\longrightarrow} \operatorname{Gr}\left(2, V_{5} / V_{1}\right)$ be the Stein factorization of $\tilde{\rho}_{2}$. As in the proof of Proposition 4.7, we see that $g$ is birational and $h$ is the double cover branched along $Z_{A} \cap \operatorname{Gr}\left(2, V_{5} / V_{1}\right)$.

The same argument shows that in the Stein factorization $\tilde{\rho}_{2}^{\prime}: \widetilde{X}^{\prime} \stackrel{g^{\prime}}{\longrightarrow} \bar{X}^{\prime} \stackrel{h^{\prime}}{\longrightarrow} \operatorname{Gr}\left(2, V_{5} / V_{1}\right)$, the map $g^{\prime}$ is also birational and $h^{\prime}$ is again the double covering branched along the same quartic 


\section{Gushel-Mukai VARIETIES}

$Z_{A} \cap \operatorname{Gr}\left(2, V_{5} / V_{1}\right)$ (Lemma B.10). As the branch divisors coincide, $\bar{X}$ is isomorphic to $\bar{X}^{\prime}$; hence $\widetilde{X}$ and $\widetilde{X}^{\prime}$ are birationally isomorphic. By Lemma 4.12, we also have a birational isomorphism between $X$ and $X^{\prime}$.

Remark 4.14. Again, one can explicitly describe the resulting birational transformation. The map $\widetilde{X} \rightarrow X$ is the blow-up of a $\sigma$-conic $c=X \cap \operatorname{CGr}\left(1, V_{5} / V_{1}\right)$, and the map $\widetilde{X} \rightarrow \bar{X}$ contracts all lines intersecting $c$ (they are parameterized by the curve $Z_{A}^{\geqslant 2} \cap \operatorname{Gr}\left(2, V_{5} / v^{\prime}\right)$ ) as well as all conics intersecting $c$ twice (these conics are $\rho$-conics and correspond to points of $\Sigma_{2}(X) \cap \operatorname{Gr}\left(2, V_{5} / V_{1}\right)$ ). The second half of the transformation is analogous.

\subsection{Combined birationalities}

In this section, we combine birational isomorphisms from the previous sections to show that all period partners and all dual varieties of any dimension $n \geqslant 3$ are birationally isomorphic. We first deal with period partners.

TheOrem 4.15. Any two smooth complex GM fourfolds which are period partners are birationally isomorphic.

Proof. Let $X_{1}$ and $X_{2}$ be smooth GM fourfolds obtained from the same Lagrangian subspace $A$ with no decomposable vectors, possibly different hyperplanes $V_{5}^{1} \subset V_{6}$ and $V_{5}^{2} \subset V_{6}$, and possibly different subspaces $A_{1}^{1}$ and $A_{1}^{2}$. By Theorem 3.27, smooth ordinary GM fourfolds that are dual to both $X_{1}$ and $X_{2}$ are parametrized by points $V_{1}$ of the locus $Y_{A}^{1}$ defined in (B.2). If we moreover impose conditions (4.4) and (4.6) for both $V_{5}^{1}$ and $V_{5}^{2}$, we need to take $V_{1}$ in $Y_{A}^{1} \cap \mathbf{P}\left(V_{5}^{1} \cap V_{5}^{2}\right)$, but not in $\Sigma_{1}\left(X_{1}\right) \cup \Sigma_{1}\left(X_{2}\right)$ (which has dimension at most 1 by Lemma 4.4). If $Y_{A} \cap \mathbf{P}\left(V_{5}^{1} \cap V_{5}^{2}\right)$ has dimension at least 3 , this is certainly possible; if it has dimension 2 , it is a surface of degree 6 , whereas $Y_{A}^{\geqslant 2}$ is an integral surface of degree 40 (Theorem B.2), so it is again possible.

We make this choice of $V_{1}$, and we let $X^{\prime}$ be the corresponding GM fourfold, dual to both $X_{1}$ and $X_{2}$. By the choice of $V_{1}$, condition (4.4) holds for both pairs $\left(X_{1}, X^{\prime}\right)$ and $\left(X_{2}, X^{\prime}\right)$; we also have $V_{1} \notin \Sigma_{1}\left(X_{1}\right) \cup \Sigma_{1}\left(X_{2}\right) \cup Y_{A}^{\geqslant 3}$ by construction. Moreover, for each $i \in\{1,2\}$, we have $\left(V_{5}^{i}\right)^{\perp} \notin \Sigma_{1}\left(X^{\prime}\right)$ (by Lemma 4.4, $\left(V_{5}^{i}\right)^{\perp} \in \Sigma_{1}\left(X^{\prime}\right)$ implies $\left(V_{1}, V_{5}^{i}\right) \in \widehat{Y}_{A}$; hence $V_{1} \in \Sigma_{1}\left(X_{i}\right)$ ) and $V_{5}^{i} \notin Y_{A^{\perp}}^{\geqslant 3}$, because $X_{1}$ and $X_{2}$ are fourfolds. Thus condition (4.6) also holds for both pairs. Applying Proposition 4.13, we conclude that $X_{1}$ and $X^{\prime}$ are birationally isomorphic, and so are $X_{2}$ and $X^{\prime}$. This proves the theorem.

Corollary 4.16. Smooth complex period partners of the same dimension $n \geqslant 3$ are birationally isomorphic.

Proof. For $n=3$, this is Theorem 4.7; for $n=4$, this is Theorem 4.15; for $n \geqslant 5$, this is Proposition 4.2.

The following corollary was brought to our attention by Tommaso de Fernex.

Corollary 4.17. No smooth complex GM variety $X$ of dimension $n \geqslant 3$ is birationally rigid: there are smooth prime Fano varieties that are birationally isomorphic to, but not biregularly isomorphic to, $X$.

Proof. By Theorem 3.25, there is a bijection between the set of isomorphism classes of period partners of $X$ and the set $\left(Y_{A^{\perp}}^{5-n} \sqcup Y_{A^{\perp}}^{6-n}\right) / \operatorname{PGL}\left(V_{6}\right)_{A}$. Since the group PGL $\left(V_{6}\right)_{A}$ is finite (Theorem B.9(a)), these sets are infinite. By Corollary 4.16, all these period partners of $X$ are birationally isomorphic to $X$. This proves the corollary. 


\section{O. Debarre And A. KuZnetsov}

For threefolds, the assumption (4.6) is very restrictive; for instance, it rules out all ordinary dual varieties. We replace it with the more flexible assumption

$$
V_{1} \in\left(Y_{A}^{2} \cap \mathbf{P}\left(V_{5}\right)\right) \backslash \Sigma_{1}(X) .
$$

The scheme $\tilde{X} \subset \mathbf{P}_{X}\left(V_{5} / \mathscr{U}_{X}\right)$ discussed in Lemma 4.12 is then reducible. We describe its irreducible components and the restriction of the map $\tilde{\rho}_{2}$ to these components.

Lemma 4.18. Let $X$ be a smooth ordinary $G M$ threefold, and let $V_{1} \subset V_{5}$ be a subspace such that (4.7) holds. The scheme $\widetilde{X}$ in (4.5) has two irreducible components: the proper preimage $\widetilde{X}_{1}$ of $X$ and the total preimage $\widetilde{X}_{2}$ of a particular line $M \subset X$. The restriction of the map $\tilde{\rho}_{2}$ to $\widetilde{X}_{2}$ is a birational isomorphism onto a Schubert hyperplane section of $\operatorname{Gr}\left(2, V_{5} / V_{1}\right)$.

Proof. The proof of Lemma 4.12 shows that the locus of non-trivial fibers of the projection $\widetilde{X} \rightarrow X$ is $X \cap \operatorname{Gr}\left(1, V_{5} / V_{1}\right)$, that is, the intersection in $\operatorname{Gr}\left(1, V_{5} / V_{1}\right)=\mathbf{P}^{3}$ of two hyperplanes and a quadric. Since $V_{1} \notin \Sigma_{1}(X)$, the intersection of the hyperplanes is a line, which we denote by $M$. Its intersection with the remaining quadric can be identified with the fiber of the first quadric fibration over the point $V_{1} \in \mathbf{P}\left(V_{5}\right)$. Therefore, under condition (4.7), its corank is 2 ; hence it coincides with $M$. Since the non-trivial fibers of the projection $\widetilde{X} \rightarrow X$ are $\mathbf{P}^{2}$, the preimage of $M$ in $\widetilde{X}$ has dimension 3 , hence is an irreducible component of $\widetilde{X}$, which we denote by $\widetilde{X}_{2}$. The other irreducible component is the proper preimage $\widetilde{X}_{1}$ of $X$ in $\tilde{X}$.

Since $M$ is a line in $\operatorname{Gr}\left(1, V_{5} / V_{1}\right)=\mathbf{P}\left(V_{5} / V_{1}\right)$, it can be written, as a subvariety of $\operatorname{Gr}\left(2, V_{5}\right)$, as $M=\left\{U_{2} \in \operatorname{Gr}\left(2, V_{5}\right) \mid V_{1} \subset U_{2} \subset V_{3}\right\}$ for some 3-dimensional subspace $V_{3} \subset V_{5}$ containing $V_{1}$. From the definition of the fibers of the map $\widetilde{X} \rightarrow X$, we get

$$
\widetilde{X}_{2} \simeq\left\{\left(U_{2}, U_{3}\right) \in \mathrm{FI}\left(2,3 ; V_{5}\right) \mid V_{1} \subset U_{2} \subset V_{3} \text { and } U_{2} \subset U_{3} \subset V_{5}\right\} .
$$

The map $\tilde{\rho}_{2}: \tilde{X} \rightarrow \operatorname{Gr}\left(2, V_{5} / V_{1}\right) \subset \operatorname{Gr}\left(3, V_{5}\right)$ takes a point $\left(U_{2}, U_{3}\right) \in \widetilde{X}_{2}$ to $U_{3} / V_{1} \in \operatorname{Gr}\left(2, V_{5} / V_{1}\right)$, hence maps $\tilde{X}_{2}$ birationally onto the Schubert hyperplane section of $\operatorname{Gr}\left(2, V_{5} / V_{1}\right)$ of all 2dimensional subspaces intersecting $V_{3} / V_{1}$ non-trivially (in fact, $\widetilde{X}_{2}$ is a Springer-type resolution of singularities of the Schubert hyperplane).

We now show that under assumption (4.7), we still have birational threefolds.

Proposition 4.19. Let $X$ and $X^{\prime}$ be dual smooth ordinary complex GM threefolds. Assume that $V_{1}^{\prime}$ satisfies (4.7) for $X$ and that $V_{5}^{\perp}$ satisfies (4.7) for $X^{\prime}$. Then $X$ and $X^{\prime}$ are birationally isomorphic.

Proof. Let $\widetilde{X}=\widetilde{X}_{1} \cup \widetilde{X}_{2}$ and $\widetilde{X}^{\prime}=\widetilde{X}_{1}^{\prime} \cup \widetilde{X}_{2}^{\prime}$ be the decompositions into irreducible components of Lemma 4.18. Consider the maps $\tilde{\rho}_{2}$ and $\tilde{\rho}_{2}^{\prime}$ outside of $\left(\Sigma_{2}(X) \cup \Sigma_{2}\left(X^{\prime}\right)\right) \cap \operatorname{Gr}\left(2, V_{5} / V_{1}^{\prime}\right)$ (this locus is 2-dimensional (Lemma 4.9), so it does not affect the birational geometry of $X$ and $X^{\prime}$ ).

Over a point of $\operatorname{Gr}\left(2, V_{5} / V_{1}^{\prime}\right)$ not in $\Sigma_{2}(X)$, the fiber of $\widetilde{X}$ is, by Proposition 4.10, a quadric in $\mathbf{P}^{0}$. It is non-empty if and only if the corresponding quadratic form is zero, that is, if its kernel is non-zero. By Proposition 4.10 again, this happens precisely for points of $Z_{A} \cap \operatorname{Gr}\left(2, V_{5} / V_{1}^{\prime}\right)$. This means that away from $\Sigma_{2}(X) \cap \operatorname{Gr}\left(2, V_{5} / V_{1}^{\prime}\right)$, the map $\tilde{\rho}_{2}$ is an isomorphism from $\tilde{X}$ onto $Z_{A} \cap \operatorname{Gr}\left(2, V_{5} / V_{1}^{\prime}\right)$. Analogously, away from $\Sigma_{2}\left(X^{\prime}\right) \cap \operatorname{Gr}\left(2, V_{5} / V_{1}^{\prime}\right)$, the map $\tilde{\rho}_{2}^{\prime}$ is an isomorphism from $\tilde{X}^{\prime}$ onto $Z_{A} \cap \operatorname{Gr}\left(2, V_{5} / V_{1}^{\prime}\right)$.

By Lemma 4.18, the components $\widetilde{X}_{2}$ and $\widetilde{X}_{2}^{\prime}$ map birationally onto the Schubert hyperplanes of $\operatorname{Gr}\left(2, V_{5} / V_{1}^{\prime}\right)$ given by the 3-dimensional spaces $V_{3} \subset V_{6}$ and $V_{3}^{\prime} \subset V_{6}^{\vee}$ corresponding to the lines $M_{V_{1}^{\prime}} \subset X$ and $M_{V_{5}^{\perp}} \subset X^{\prime}$, respectively. Let us show $V_{3}^{\prime}=V_{3}^{\perp}$, so that the Schubert hyperplanes coincide. 


\section{Gushel-Mukai VARIETIES}

By the definition of the line $M_{V_{1}^{\prime}}$, the space $V_{3}$ can be constructed as follows. Let

$$
A \cap\left(V_{1}^{\prime} \wedge \wedge^{2} V_{6}\right)=\left\langle v \wedge \xi_{1}, v \wedge \xi_{2}\right\rangle,
$$

where $v$ is a generator of $V_{1}^{\prime}$ and $\xi_{1}, \xi_{2} \in \Lambda^{2} V_{6}$. Let $f \in V_{6}^{\vee}$ be an equation of $V_{5}$. Then $V_{3}$ is spanned by $v$ and the contractions $\xi_{i}(f,-)$. Analogously, if

$$
A^{\perp} \cap\left(V_{5}^{\perp} \wedge \wedge^{2} V_{6}^{\vee}\right)=\left\langle f \wedge \eta_{1}, f \wedge \eta_{2}\right\rangle,
$$

the space $V_{3}^{\prime}$ is spanned by $f$ and the contractions $\eta_{i}(v,-)$ of $\eta_{i} \in \Lambda^{2} V_{6}^{\vee}$. To identify $V_{3}^{\prime}$ with $V_{3}^{\perp}$, it is therefore enough to show

$$
\left(\xi_{i}(f,-), \eta_{j}(v,-)\right)=0
$$

for all $i, j \in\{1,2\}$. This follows from the equality

$$
\left(v \wedge \xi_{i}, f \wedge \eta_{j}\right)=\left(\xi_{i}(f,-), \eta_{j}(v,-)\right),
$$

which in turn follows from $f(v)=0$.

We proved that $\widetilde{X}_{2}$ and $\widetilde{X}_{2}^{\prime}$ are mapped by $\tilde{\rho}_{2}$ and $\tilde{\rho}_{2}^{\prime}$ onto the same Schubert hyperplane section $H \subset \operatorname{Gr}\left(2, V_{5} / V_{1}^{\prime}\right)$. Since both $\widetilde{X}$ and $\widetilde{X}^{\prime}$ map birationally onto the quartic hypersurface $Z_{A} \cap \operatorname{Gr}\left(2, V_{5} / V_{1}^{\prime}\right)$, it follows that this quartic has two components $H$ and $Z^{\prime}$, where $Z^{\prime}$ is a cubic hypersurface in $\operatorname{Gr}\left(2, V_{5} / V_{1}^{\prime}\right)$ onto which both $\widetilde{X}_{1}$ and $\widetilde{X}_{1}^{\prime}$ map birationally. Thus $\widetilde{X}_{1}$ is birational to $\widetilde{X}_{1}^{\prime}$, hence $X$ is birational to $X^{\prime}$.

The birational isomorphism of Proposition 4.19 coincides with the transformation of $X$ (with respect to the line $M$ ) defined in [DIM12, Section 7.2].

We can now prove the main result of this section.

Theorem 4.20. Any two dual smooth complex GM varieties of the same dimension $n \geqslant 3$ are birationally isomorphic.

Proof. We keep the same notation as above. For $n \geqslant 5$, the result is trivial by Proposition 4.2, so we may assume $n \in\{3,4\}$.

First, assume $n=4$. As in the proof of Theorem 4.15, we may choose $V_{1}^{\prime \prime} \subset V_{5}$ such that the corresponding smooth GM fourfold $X^{\prime \prime}$ is dual to $X$. Then $X$ is birationally isomorphic to $X^{\prime \prime}$ by Proposition 4.13, and $X^{\prime}$ and $X^{\prime \prime}$ are birationally isomorphic by Theorem 4.15; hence $X$ is birationally isomorphic to $X^{\prime}$.

Now, let $X$ be a smooth GM threefold, and set $A:=A(X)$. By Lemma B.5, we can find $v \in \mathbf{P}\left(V_{6}\right)$ and $V_{5} \subset V_{6}$ such that

$$
v \in Y_{A}^{2}, \quad\left[V_{5}\right] \in Y_{A^{\perp}}^{2}, \quad v \in V_{5}, \quad \text { and } \quad\left(v, V_{5}\right) \notin(p \times q)\left(\widehat{Y}_{A}\right) .
$$

Let $X^{\prime}$ be the ordinary GM threefold corresponding to the Lagrangian $A$ and the Plücker hyperplane $V_{5} \subset V_{6}$, and let $X^{\prime \prime}$ be the ordinary GM threefold corresponding to the Lagrangian $A^{\perp}$ and the Plücker hyperplane $v^{\perp} \subset V_{6}^{\vee}$.

Then $X^{\prime}$ is a period partner of $X$; hence $X^{\prime}$ is birational to $X$ by Theorem 4.7. Furthermore, $X^{\prime \prime}$ is dual to $X^{\prime}$, and the conditions of Proposition 4.19 are satisfied for the pair $\left(X^{\prime}, X^{\prime \prime}\right)$ (the last condition in (4.8) is equivalent to $v \notin \Sigma_{1}\left(X^{\prime}\right)$ by Lemma 4.4). Therefore, $X^{\prime \prime}$ is birational to $X^{\prime}$ by Proposition 4.19. Combining these two birationalities, we conclude that $X^{\prime \prime}$ is birational to $X$. It remains to note that any other dual GM threefold of $X$ is a period partner of $X^{\prime \prime}$, hence is birational to $X^{\prime \prime}$ by Theorem 4.7. 


\section{O. Debarre And A. KuZnetsov}

\section{Appendix A. Excess conormal sheaves}

Let $W$ be a k-vector space, and let $X \subset \mathbf{P}(W)$ be a closed $\mathbf{k}$-subscheme which is an intersection of quadrics; that is, the twisted ideal sheaf $\mathscr{I}_{X}(2)$ on $\mathbf{P}(W)$ is globally generated. Let

$$
V_{X}:=H^{0}\left(\mathbf{P}(W), \mathscr{I}_{X}(2)\right)
$$

be the space of quadrics through $X$. The canonical map $V_{X} \otimes \mathscr{O}_{\mathbf{P}(W)}(-2) \rightarrow \mathscr{I}_{X}$ is surjective, and its restriction to $X$ induces an epimorphism $V_{X} \otimes \mathscr{O}_{X}(-2) \rightarrow \mathscr{I}_{X} / \mathscr{I}_{X}^{2}=\mathscr{N}_{X / \mathbf{P}(W)}^{\vee}$ onto the conormal sheaf of $X$.

Definition A.1. The excess conormal sheaf $\mathscr{E} \mathscr{N}_{X / \mathbf{P}(W)}^{\vee}$ of an intersection of quadrics $X \subset \mathbf{P}(W)$ is the kernel of the canonical map $V_{X} \otimes \mathscr{O}_{X}(-2) \rightarrow \mathscr{N}_{X / \mathbf{P}(W)}^{\vee}$.

When the ambient space $\mathbf{P}(W)$ is clear, we will simply write $\mathscr{E} \mathscr{N}_{X}^{\vee}$. This sheaf is defined over $\mathbf{k}$ and fits in the excess conormal sequence

$$
0 \rightarrow \mathscr{E}_{X / \mathbf{N}(W)}^{\vee} \rightarrow V_{X} \otimes \mathscr{O}_{X}(-2) \rightarrow \mathscr{N}_{X / \mathbf{P}(W)}^{\vee} \rightarrow 0
$$

We will also often consider the twist

$$
0 \rightarrow \mathscr{E} \mathscr{N}_{X / \mathbf{P}(W)}^{\vee}(2) \rightarrow V_{X} \otimes \mathscr{O}_{X} \rightarrow \mathscr{N}_{X / \mathbf{P}(W)}^{\vee}(2) \rightarrow 0
$$

In this appendix, we discuss some properties of the excess conormal sheaf.

Lemma A.2. The excess conormal sheaf is locally free on the lci locus of $X$.

Proof. This follows from the local freeness of the conormal bundle on the lci locus of $X$.

Proposition A.3. Consider a closed subscheme $X \subset \mathbf{P}(W)$ and a cone $\mathrm{C}_{K} X \subset \mathbf{P}(W \oplus K)$. Let $W^{\prime} \subset W \oplus K$ be a vector subspace, and let $X^{\prime} \subset \mathbf{P}\left(W^{\prime}\right) \cap C_{K} X$ be a closed subscheme. Set $X_{0}^{\prime}:=X^{\prime} \cap C_{K}^{\circ} X$. If both $X$ and $X^{\prime}$ are intersections of quadrics, there is a canonical commutative diagram

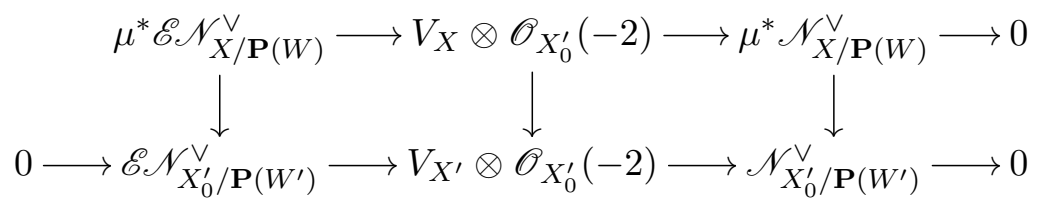

of sheaves on $X_{0}^{\prime}$, where $\mu$ denotes the natural projection $\mathbf{P}(W \oplus K) \backslash \mathbf{P}(K)=\mathrm{C}_{K}^{\circ} \mathbf{P}(W) \rightarrow \mathbf{P}(W)$ as well as its restriction to $X_{0}^{\prime}$.

Proof. The embedding $W^{\prime} \subset W \oplus K$ induces a map $\mathrm{S}^{2} W^{\vee} \rightarrow \mathrm{S}^{2} W^{\prime \vee}$. Denote by $V^{\prime}$ the image of $V_{X}$ under this map. Clearly, $\mathbf{P}\left(W^{\prime}\right) \cap \mathrm{C}_{K} X$ is cut out in $\mathbf{P}\left(W^{\prime}\right)$ by the quadrics in $V^{\prime}$. Hence the space $V_{X^{\prime}}$ of quadrics cutting out $X^{\prime}$ in $\mathbf{P}\left(W^{\prime}\right)$ contains $V^{\prime}$, and we obtain a canonical map $V_{X} \rightarrow V_{X^{\prime}}$. Furthermore, on the open subset $\mathbf{P}\left(W^{\prime}\right) \backslash \mathbf{P}(K) \subset \mathbf{P}\left(W^{\prime}\right)$, the pullback $\mu^{*} \mathscr{I}_{X}$ of the ideal of $X$ in $\mathbf{P}(W)$ is contained in the ideal $\mathscr{I}_{X_{0}^{\prime}}$ of $X_{0}^{\prime}$ in $\mathbf{P}\left(W^{\prime}\right) \backslash \mathbf{P}(K)$; hence we have a commutative diagram

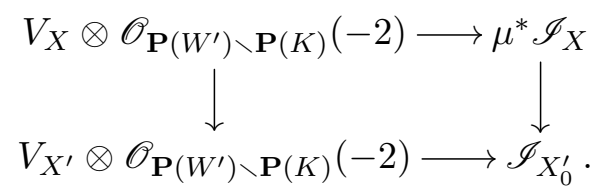

Restricting it to $X_{0}^{\prime}$ and extending lines to excess conormal sequences proves the claim.

In some cases, the vertical map in (A.2) between excess conormal sheaves is an isomorphism. 


\section{Gushel-Mukai VARIETIES}

Lemma A.4. In the situation of Proposition A.3, assume $W^{\prime}=W \oplus \mathbf{k}$ and $X^{\prime}=\mathrm{C}_{\mathbf{k}} X \subset \mathbf{P}\left(W^{\prime}\right)$, so that $X_{0}^{\prime}=\mathrm{C}_{\mathbf{k}}^{\circ} X$ is the punctured cone. If $\mu: X_{0}^{\prime} \rightarrow X$ is the natural projection, we have $\left.\mathscr{E} \mathscr{N}_{X^{\prime}}^{\vee}\right|_{X_{0}^{\prime}} \simeq \mu^{*} \mathscr{E} \mathscr{N}_{X}^{\vee}$

Proof. The intersection in $\mathbf{P}\left(W^{\prime}\right)$ of the quadrics in $V_{X} \subset \mathrm{S}^{2} W^{\vee} \subset \mathrm{S}^{2} W^{\prime \vee}$ is the cone $X^{\prime}=\mathrm{C}_{\mathbf{k}} X$. Moreover, $\mathscr{N}_{X_{0}^{\prime} / \mathbf{P}\left(W^{\prime}\right)} \simeq \mu^{*} \mathscr{N}_{X / \mathbf{P}(W)}$. Thus, the central and right vertical arrows in (A.2) are isomorphisms. Moreover, the map $\mu$ is flat; hence the top line is exact on the left. Therefore the left arrow is an isomorphism as well.

Lemma A.5. Assume that $X \subset \mathbf{P}(W)$ is an intersection of quadrics and is linearly normal. Let $W^{\prime} \subset W$ be a hyperplane such that $X^{\prime}:=X \cap \mathbf{P}\left(W^{\prime}\right)$ is a dimensionally transverse intersection. Then $X^{\prime} \subset \mathbf{P}\left(W^{\prime}\right)$ is an intersection of quadrics and $\left.\mathscr{E} \mathscr{N}_{X_{\mathrm{lci}}^{\prime}}^{\vee} \simeq \mathscr{E} \mathscr{N}_{X_{\mathrm{lci}}}^{\vee}\right|_{X_{\mathrm{lci}}^{\prime}}$, where $X_{\text {lci }}$ is the lci locus of $X$ and $X_{\mathrm{lci}}^{\prime}=X^{\prime} \cap X_{\mathrm{lci}}$.

Proof. We have an exact sequence

$$
0 \rightarrow \mathscr{I}_{X}(-1) \rightarrow \mathscr{I}_{X} \rightarrow \mathscr{I}_{X^{\prime} / \mathbf{P}\left(W^{\prime}\right)} \rightarrow 0
$$

Twisting it by $\mathscr{O}_{\mathbf{P}(W)}(2)$ and using the linear normality of $X$, we conclude that $X^{\prime}$ is an intersection of quadrics and $V_{X^{\prime}}=V_{X}$. The diagram of Proposition A.3 gives a commutative diagram

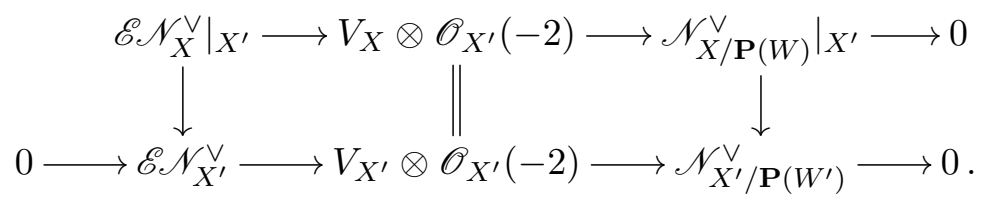

The right vertical arrow is an isomorphism by the dimensionally transverse intersection condition. Moreover, the top line is exact on the lci locus, since the conormal bundle is locally free. Hence the left arrow is an isomorphism.

Lemma A.6. Assume $H^{0}\left(X, \mathscr{O}_{X}\right)=\mathbf{k}$, and let $Q \subset \mathbf{P}(W)$ be a quadratic hypersurface such that $X^{\prime}=X \cap Q$ is a dimensionally transverse intersection. Then $\left.\mathscr{E}_{X_{\mathrm{lci}}^{\prime}}^{\vee} \simeq \mathscr{E} \mathscr{N}_{X_{\mathrm{lci}}}^{\vee}\right|_{X_{\mathrm{lci}}^{\prime}}$, where again $X_{\text {lci }}$ is the lci locus of $X$ and $X_{\text {lci }}^{\prime}=X^{\prime} \cap X_{\text {lci }}$.

Proof. Tensoring the exact sequences

$$
0 \rightarrow \mathscr{I}_{X} \rightarrow \mathscr{O}_{\mathbf{P}(W)} \rightarrow \mathscr{O}_{X} \rightarrow 0 \quad \text { and } \quad 0 \rightarrow \mathscr{O}_{\mathbf{P}(W)}(-2) \rightarrow \mathscr{O}_{\mathbf{P}(W)} \rightarrow \mathscr{O}_{Q} \rightarrow 0
$$

and taking into account the dimensional transversality of the intersection $X^{\prime}=X \cap Q$, we obtain an exact sequence

$$
0 \rightarrow \mathscr{I}_{X}(-2) \rightarrow \mathscr{I}_{X} \oplus \mathscr{O}_{\mathbf{P}(W)}(-2) \rightarrow \mathscr{I}_{X^{\prime}} \rightarrow 0
$$

Twisting it by $\mathscr{O}_{\mathbf{P}(W)}(2)$ and taking into account that the condition $H^{0}\left(X, \mathscr{O}_{X}\right)=\mathbf{k}$ implies $H^{0}\left(\mathbf{P}(W), \mathscr{I}_{X}\right)=H^{1}\left(\mathbf{P}(W), \mathscr{I}_{X}\right)=0$, we obtain an isomorphism $V_{X^{\prime}}=V_{X} \oplus \mathbf{k}$. It follows that 


\section{O. Debarre And A. KuZnetsov}

the diagram of Proposition A.3 extends to a commutative diagram

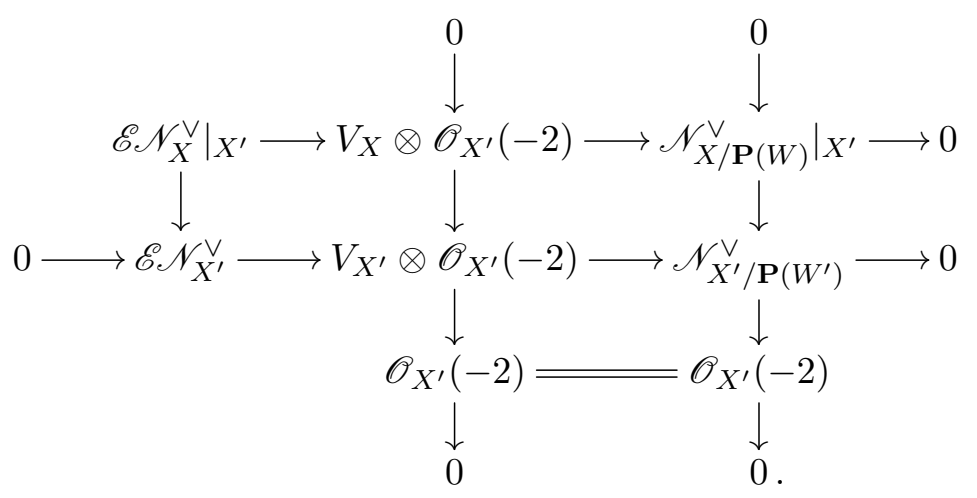

Its middle column is exact by the above argument, and the right column is exact by the dimensionally transverse intersection condition. Moreover, the top line is exact on $X_{\text {lci }}$; hence the left vertical arrow is an isomorphism.

Finally, we compute the excess conormal bundle for some examples. Let $\mathscr{U}$ be the rank 2 tautological vector bundle on the Grassmannian $\operatorname{Gr}\left(2, V_{5}\right)$.

Proposition A.7. Let $X=\operatorname{Gr}\left(2, V_{5}\right) \subset \mathbf{P}\left(\bigwedge^{2} V_{5}\right)$. Then $\mathscr{E}_{X}^{\vee} \simeq \operatorname{det}\left(V_{5}^{\vee}\right) \otimes \mathscr{U}(-2)$.

Proof. The standard resolution of the structure sheaf $\mathscr{O}_{X}$ in $\mathbf{P}\left(\bigwedge^{2} V_{5}\right)$ is

$0 \rightarrow \operatorname{det}\left(V_{5}^{\vee}\right)^{\otimes 2} \otimes \mathscr{O}(-5) \rightarrow \operatorname{det}\left(V_{5}^{\vee}\right) \otimes V_{5}^{\vee} \otimes \mathscr{O}(-3) \stackrel{\alpha}{\longrightarrow} \operatorname{det}\left(V_{5}^{\vee}\right) \otimes V_{5} \otimes \mathscr{O}(-2) \rightarrow \mathscr{O} \rightarrow \mathscr{O}_{X} \rightarrow 0$,

where the map $\alpha$ is induced by the contraction $V_{5}^{\vee} \otimes \bigwedge^{2} V_{5} \rightarrow V_{5}$. In particular, the space $\operatorname{det}\left(V_{5}^{\vee}\right) \otimes V_{5}=\bigwedge^{4} V_{5}^{\vee}$ can be identified with the space of quadrics through $X$. Tensoring this resolution with $\mathscr{O}_{X}$ and taking into account that $\operatorname{Tor}_{i}\left(\mathscr{O}_{X}, \mathscr{O}_{X}\right)=\bigwedge^{i} \mathscr{N}_{X}^{\vee}$, we deduce an exact sequence

$$
0 \rightarrow \bigwedge^{2} \mathscr{N}_{X}^{\vee} \rightarrow \operatorname{det}\left(V_{5}^{\vee}\right) \otimes V_{5}^{\vee} \otimes \mathscr{O}_{X}(-3) \stackrel{\left.\alpha\right|_{X}}{\longrightarrow} \operatorname{det}\left(V_{5}^{\vee}\right) \otimes V_{5} \otimes \mathscr{O}_{X}(-2) \rightarrow \mathscr{N}_{X}^{\vee} \rightarrow 0 .
$$

The above description of $\alpha$ shows that $\left.\alpha\right|_{X}$ is the twist by $\operatorname{det}\left(V_{5}^{\vee}\right)$ of the composition of the epimorphism $V_{5}^{\vee} \otimes \mathscr{O}_{X}(-3) \rightarrow \mathscr{U}^{\vee}(-3)$, an isomorphism $\mathscr{U}^{\vee}(-3) \stackrel{\sim}{\longrightarrow} \mathscr{U}(-2)$, and the monomorphism $\mathscr{U}(-2) \hookrightarrow V_{5} \otimes \mathscr{O}_{X}(-2)$, whence the claim.

Let $V_{6}$ be a $\mathbf{k}$-vector space of dimension 6 , and let again $\mathscr{U}$ be the rank 2 tautological vector bundle on the Grassmannian $\operatorname{Gr}\left(2, V_{6}\right)$.

Proposition A.8. Let $X=\operatorname{Gr}\left(2, V_{6}\right) \subset \mathbf{P}\left(\bigwedge^{2} V_{6}\right)$. Then

$$
\mathscr{E} \mathscr{N}_{X}^{\vee} \simeq \operatorname{det}\left(V_{6}^{\vee}\right) \otimes\left(\left(V_{6} \otimes \mathscr{U}(-2)\right) /\left(\mathrm{S}^{2} \mathscr{U}\right)(-2)\right) .
$$

Proof. The standard resolution [Wey03, Theorem 6.4.1] of the structure sheaf of $X$ in $\mathbf{P}\left(\bigwedge^{2} V_{6}\right)$ is

$$
\cdots \rightarrow \operatorname{det}\left(V_{6}^{\vee}\right) \otimes \mathfrak{s l}\left(V_{6}\right) \otimes \mathscr{O}(-3) \stackrel{\beta}{\longrightarrow} \operatorname{det}\left(V_{6}^{\vee}\right) \otimes \bigwedge^{2} V_{6} \otimes \mathscr{O}(-2) \rightarrow \mathscr{O} \rightarrow \mathscr{O}_{X} \rightarrow 0,
$$

where $\beta$ is given by the natural Lie algebra action $\mathfrak{s l}\left(V_{6}\right) \otimes \bigwedge^{2} V_{6} \rightarrow \bigwedge^{2} V_{6}$. Tensoring this resolution with $\mathscr{O}_{X}$, we deduce an exact sequence

$$
\operatorname{det}\left(V_{6}^{\vee}\right) \otimes \mathfrak{s l}\left(V_{6}\right) \otimes \mathscr{O}_{X}(-3) \stackrel{\left.\beta\right|_{X}}{\longrightarrow} \operatorname{det}\left(V_{6}^{\vee}\right) \otimes \bigwedge^{2} V_{6} \otimes \mathscr{O}_{X}(-2) \rightarrow \mathscr{N}_{X}^{\vee} \rightarrow 0 .
$$

The above description of $\beta$ shows that $\left.\beta\right|_{X}$ is the twist by $\operatorname{det}\left(V_{6}^{\vee}\right)$ of the composition

$$
\mathfrak{s l}\left(V_{6}\right) \otimes \mathscr{O}_{X}(-3) \rightarrow V_{6} \otimes \mathscr{U}^{\vee}(-3) \stackrel{\sim}{\longrightarrow} V_{6} \otimes \mathscr{U}(-2) \rightarrow \bigwedge^{2} V_{6} \otimes \mathscr{O}_{X}(-2) .
$$




\section{Gushel-Mukai VARIETIES}

Since the first arrow is surjective, the image of the composition (that is, the excess conormal bundle of the Grassmannian) is isomorphic (up to a twist) to the image of $V_{6} \otimes \mathscr{U}(-2)$. The kernel of the last map is $\left(\mathrm{S}^{2} \mathscr{U}\right)(-2)$, whence the claim.

\section{Appendix B. Eisenbud-Popescu-Walter sextics}

These sextics were introduced by Eisenbud, Popescu, and Walter in [EPW01, Example 9.3] and were thoroughly investigated, over $\mathbf{C}$, by O'Grady in the series of articles [OGr06, OGr08a, OGr08b, OGr12, OGr13, OGr15, OGr16]. For their remarkable properties, we refer to these articles. In this appendix, we sketch the original construction of EPW sextics from Lagrangian subspaces and discuss some of their properties. The only new results here are Lemmas B.5 and B.6. To be able to use O'Grady's results, we work over $\mathbf{C}$, although many of his results are valid over more general fields.

\section{B.1 An overview of EPW sextics}

Let $V_{6}$ be a 6-dimensional complex vector space, and let $A \subset \wedge^{3} V_{6}$ be a Lagrangian subspace for the $\operatorname{det}\left(V_{6}\right)$-valued symplectic form $\omega$ defined by

$$
\omega(\xi, \eta)=\xi \wedge \eta \text {. }
$$

Definition B.1. For any integer $\ell$, we set

$$
Y_{A}^{\geqslant \ell}:=\left\{[v] \in \mathbf{P}\left(V_{6}\right) \mid \operatorname{dim}\left(A \cap\left(v \wedge \wedge^{2} V_{6}\right)\right) \geqslant \ell\right\}
$$

and endow it with a scheme structure as in [OGr06, Section 2]. The locally closed subsets

$$
Y_{A}^{\ell}:=Y_{A}^{\geqslant \ell} \backslash Y_{A}^{\geqslant \ell+1}
$$

form the EPW stratification of $\mathbf{P}\left(V_{6}\right)$, and the sequence of inclusions

$$
\mathbf{P}\left(V_{6}\right)=Y_{A}^{\geqslant 0} \supset Y_{A}^{\geqslant 1} \supset Y_{A}^{\geqslant 2} \supset \cdots
$$

is called the EPW sequence. When the scheme $Y_{A}:=Y_{A}^{\geqslant 1}$ is not the whole space $\mathbf{P}\left(V_{6}\right)$, it is a sextic hypersurface [OGr06, (1.8)] called an EPW sextic. The scheme $Y_{A}^{\geqslant 2}$ is non-empty and has everywhere dimension at least 2 [OGr06, (2.9)].

Non-zero elements of $A$ which can be written as $v_{1} \wedge v_{2} \wedge v_{3}$ are called decomposable vectors, so that $A$ contains no decomposable vectors if the scheme

$$
\Theta_{A}:=\mathbf{P}(A) \cap \operatorname{Gr}\left(3, V_{6}\right)
$$

is empty. When $\mathbf{P}\left(V_{6}\right) \neq \bigcup_{V_{3} \in \Theta_{A}} \mathbf{P}\left(V_{3}\right)$ (for example, when $\operatorname{dim}\left(\Theta_{A}\right) \leqslant 2$ ), the scheme $Y_{A}$ is not $\mathbf{P}\left(V_{6}\right)$ and its singular locus can be described [OGr12, Corollary 2.5] as

$$
\operatorname{Sing}\left(Y_{A}\right)=Y_{A}^{\geqslant 2} \cup\left(\bigcup_{V_{3} \in \Theta_{A}} \mathbf{P}\left(V_{3}\right)\right) .
$$

The following theorem gathers various results of O'Grady.

Theorem B.2 (O'Grady). Let $A \subset \wedge^{3} V_{6}$ be a Lagrangian subspace. If $A$ contains only finitely many decomposable vectors, then

(a) $Y_{A}$ is an integral normal sextic hypersurface in $\mathbf{P}\left(V_{6}\right)$;

(b) $Y_{A}^{\geqslant 2}$ is a surface; 


\section{O. Debarre and A. Kuznetsov}

(c) $Y_{A}^{\geqslant 3}$ is finite if the scheme $\Theta_{A}$ is moreover reduced;

(d) $Y_{A}^{\geqslant 5}$ is empty.

If, moreover, A contains no decomposable vectors, then

$\left(\mathrm{b}^{\prime}\right) Y_{A}^{\geqslant 2}=\operatorname{Sing}\left(Y_{A}\right)$ is an integral normal Cohen-Macaulay surface of degree 40;

(c') $Y_{A}^{\geqslant 3}=\operatorname{Sing}\left(Y_{A}^{\geqslant 2}\right)$ is finite and smooth, and is empty for A general;

(d') $Y_{A}^{\geqslant 4}$ is empty.

Note that if $A$ is the dual Lagrangian (see Section B.2) associated with a strongly smooth ordinary GM curve, $A$ contains five decomposable vectors and $Y_{A}^{\geqslant 4}$ is non-empty (Remark 3.17).

Proof. The finite union $S:=\bigcup_{V_{3} \in \Theta_{A}} \mathbf{P}\left(V_{3}\right)$ is a surface if $\Theta_{A}$ is non-empty (finite), and it is empty otherwise. Relation (B.3) holds; hence claim (a) will follow from claim (b).

As we already mentioned, the scheme $Y_{A}^{\geqslant 2}$ has everywhere dimension at least 2 [OGr06, (2.9)]. We now show that $Y_{A}^{\geqslant 2}$ has dimension at most 2 at any point $v \notin S$. If $v \in Y_{A}^{2}$, this is [OGr12, Proposition 2.9]. Assume $v \in Y_{A}^{\geqslant 3}$; the proof of [OGr13, Claim 3.7] still applies because $v \notin S$. It gives $v \in Y_{A}^{3}$ and that $v$ is isolated in $Y_{A}^{\geqslant 3}$, which proves that $Y_{A}^{\geqslant 3} \backslash S$ is finite. This implies that in any positive-dimensional component $T$ of $Y_{A}^{\geqslant 2} \backslash S$, the open subset $T \cap Y_{A}^{2}$ is dense and has dimension at most 2 . Therefore, $\operatorname{dim}(T) \leqslant 2$ and claim (b) is proved.

To finish the proof of claim (c), we show that the set $Y_{A}^{\geqslant 3} \cap \mathbf{P}\left(V_{3}\right)$ is finite for each $V_{3} \in \Theta_{A}$, under the additional assumption that $\Theta_{A}$ is reduced. Since

$$
\mathbf{T}_{\Theta_{A},\left[V_{3}\right]}=\mathbf{P}(A) \cap \mathbf{T}_{\mathrm{Gr}\left(3, V_{6}\right),\left[V_{3}\right]}=\mathbf{P}\left(A \cap\left(\bigwedge^{2} V_{3} \wedge V_{6}\right)\right) \subset \mathbf{P}\left(\bigwedge^{3} V_{6}\right),
$$

we have $A \cap\left(\bigwedge^{2} V_{3} \wedge V_{6}\right)=\bigwedge^{3} V_{3}$. In the notation of [OGr16, Definition 3.3.3], this implies

$$
\mathscr{B}\left(V_{3}, A\right)=\left\{[v] \in \mathbf{P}\left(V_{3}\right) \mid \exists V_{3}^{\prime} \in \Theta_{A}, V_{3}^{\prime} \neq V_{3} \text { and } v \in V_{3}^{\prime}\right\} \text {. }
$$

But this set is finite: if $V_{3}, V_{3}^{\prime} \in \Theta_{A}$ and $\operatorname{dim}\left(V_{3} \cap V_{3}^{\prime}\right) \geqslant 2$, the line spanned by [V $V_{3}$ ] and $\left[V_{3}^{\prime}\right]$ in $\mathbf{P}\left(\bigwedge^{3} V_{6}\right)$ is contained in $\operatorname{Gr}\left(3, V_{6}\right)$, hence in $\Theta_{A}$, contradicting the finiteness of $\Theta_{A}$. The finiteness of $Y_{A}^{\geqslant 3} \cap \mathbf{P}\left(V_{3}\right)$ then follows from [OGr16, Proposition 3.3.6] and claim (c) is proved.

Finally, assume $v \in Y_{A}^{\geqslant 5}$. Inside $\mathbf{P}\left(\bigwedge^{2}\left(V_{6} / v\right)\right)=\mathbf{P}^{9}$, the linear space $\mathbf{P}\left(A \cap\left(v \wedge \bigwedge^{2}\left(V_{6} / v\right)\right)\right)$ (of dimension at least 4 ) and the Grassmannian $\operatorname{Gr}\left(2, V_{6} / v\right)$ (of dimension 6) meet along a positivedimensional locus contained in $\Theta_{A}$, which is absurd. This proves claim (d).

For the proof of claims $\left(b^{\prime}\right),\left(c^{\prime}\right)$, and $\left(d^{\prime}\right)$, we have $\Theta_{A}=\varnothing$.

Again, we have $\operatorname{Sing}\left(Y_{A}\right)=Y_{A}^{\geqslant 2}$ by (B.3); moreover, $Y_{A}^{2}$ is smooth of the expected dimension 2 [OGr12, Proposition 2.9], so that $\operatorname{Sing}\left(Y_{A}^{\geqslant 2}\right) \subset Y_{A}^{\geqslant 3}$. On the other hand, [OGr13, Claim 3.7] says that claim $\left(\mathrm{d}^{\prime}\right)$ holds; the proof actually shows that the tangent space to $Y_{A}^{\geqslant 3}$ at any point is zero, so that $Y_{A}^{\geqslant 3}$ is smooth. It is moreover empty for $A$ general [OGr06, Claim 2.6].

All this implies that the determinantal locus $Y_{A}^{\geqslant 2}$ has everywhere the expected dimension, 2 . Therefore, it is Cohen-Macaulay and $Y_{A}^{\geqslant 3} \subset \operatorname{Sing}\left(Y_{A}^{\geqslant 2}\right)$. This completes the proof of claim $\left(\mathrm{c}^{\prime}\right)$. It also implies that $Y_{A}^{\geqslant 2}$ is normal since it is Cohen-Macaulay and its singular locus is finite. Its degree is 40 by [OGr06, (2.9)].

It remains to show that $Y_{A}^{\geqslant 2}$ is irreducible. When $A$ is general, this follows from [OGr08b, Theorem 1.1]. To deduce irreducibility for any $A$ with no decomposable vectors, we use a standard trick. Let $(T, 0)$ be a smooth, pointed, connected curve that parametrizes Lagrangians $\left(A_{t}\right)_{t \in T}$ with no decomposable vectors such that for $t \in T$ general, $Y_{A_{t}}^{\geqslant 2}$ is smooth irreducible and $A_{0}=A$. 


\section{Gushel-Mukai VARIETIES}

Let $H \subset \mathbf{P}\left(V_{6}\right)$ be a general hyperplane, so that $H \cap Y_{A_{0}}^{\geqslant 2}$ is a smooth curve and $H \cap Y_{A_{t}}^{\geqslant 2}$ is smooth irreducible for general $t$. In $T \times \mathbf{P}^{5}$, consider the union of all surfaces $\{t\} \times Y_{A_{t}}^{\geqslant 2}$. As a determinantal locus, it has everywhere codimension at most 3 ; hence its intersection $\mathscr{Y}$ with $T \times H$ has everywhere dimension at least 2 . Since the fiber $\mathscr{Y}_{0}=H \cap Y_{A_{0}}^{\geqslant 2}$ is 1-dimensional, any component of $\mathscr{Y}_{0}$ deforms to a neighborhood of 0 in $T$. Since $\mathscr{Y}_{t}$ is irreducible for $t$ general, so is $\mathscr{Y}_{0}$, hence so is $Y_{A_{0}}^{\geqslant 2}$. This finishes the proof of the theorem.

\section{B.2 The duality of EPW sextics}

If $A \subset \bigwedge^{3} V_{6}$ is a Lagrangian subspace, its orthogonal $A^{\perp} \subset \bigwedge^{3} V_{6}^{\vee}$ is also a Lagrangian subspace. In the dual projective space $\mathbf{P}\left(V_{6}^{\vee}\right)=\operatorname{Gr}\left(5, V_{6}\right)$, the EPW sequence for $A^{\perp}$ can be described in terms of $A$ as

$$
Y_{A^{\perp}}^{\geqslant \ell}=\left\{V_{5} \in \operatorname{Gr}\left(5, V_{6}\right) \mid \operatorname{dim}\left(A \cap \bigwedge^{3} V_{5}\right) \geqslant \ell\right\} .
$$

The canonical identification $\operatorname{Gr}\left(3, V_{6}\right) \simeq \operatorname{Gr}\left(3, V_{6}^{\vee}\right)$ induces an isomorphism between the scheme $\Theta_{A}$ of decomposable vectors in $\mathbf{P}(A)$ and the scheme $\Theta_{A^{\perp}}$ of decomposable vectors in $\mathbf{P}\left(A^{\perp}\right)$ [OGr12, (2.82)]. In particular, $A$ contains no decomposable vectors if and only if the same is true for $A^{\perp}$.

One of the interesting properties of an EPW sextic is that its projective dual is often also an EPW sextic. The proof we give is essentially equivalent to O'Grady's [OGr08a, Corollary 3.6] but is written in more geometrical terms.

Consider the scheme

$$
\widehat{Y}_{A}:=\left\{\left(v, V_{5}\right) \in \operatorname{Fl}\left(1,5 ; V_{6}\right) \mid A \cap\left(v \wedge \wedge^{2} V_{5}\right) \neq 0\right\}
$$

and the projections

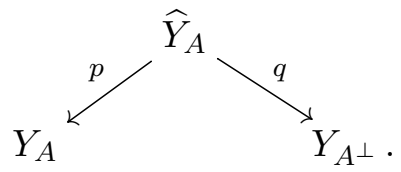

Proposition B.3. If the scheme $\Theta_{A}$ of decomposable vectors in $\mathbf{P}(A)$ is finite and reduced, the hypersurfaces $Y_{A} \subset \mathbf{P}\left(V_{6}\right)$ and $Y_{A^{\perp}} \subset \mathbf{P}\left(V_{6}^{\vee}\right)$ are projectively dual. If $A$ contains no decomposable vectors, $\widehat{Y}_{A}$ is irreducible and realizes the projective duality between these two hypersurfaces.

Proof. We keep the notation $S=\bigcup_{V_{3} \in \Theta_{A}} \mathbf{P}\left(V_{3}\right)$ and define

$$
\widehat{Y}_{A}^{\prime}:=\left\{\left(a, v, V_{5}\right) \in \mathbf{P}(A) \times \operatorname{FI}\left(1,5 ; V_{6}\right) \mid a \in \mathbf{P}\left(A \cap\left(v \wedge \wedge^{2} V_{5}\right)\right)\right\},
$$

with the forgetful map $\widehat{Y}_{A}^{\prime} \rightarrow \widehat{Y}_{A}$ and the maps $p^{\prime}: \widehat{Y}_{A}^{\prime} \rightarrow \widehat{Y}_{A} \stackrel{p}{\rightarrow} Y_{A}$ and $q^{\prime}: \widehat{Y}_{A}^{\prime} \rightarrow \widehat{Y}_{A} \stackrel{q}{\rightarrow} Y_{A^{\perp}}$.

Let us determine the fiber of $p^{\prime}$ over $v \in Y_{A}$. Take $a \in A \cap\left(v \wedge \wedge^{2} V_{6}\right)$ non-zero, and write $a=v \wedge \eta$ with $\eta \in \wedge^{2}\left(V_{6} / v\right)$.

If $a$ is not decomposable, so is $\eta$; hence its rank is 4 , and there is a unique hyperplane $V_{5} \subset V_{6}$ containing $v$ such that $\eta \in \bigwedge^{2}\left(V_{5} / v\right)$. Moreover, the space $V_{5}$ corresponding to $a=v \wedge \eta$ is given by the 4 -form

$$
\eta \wedge \eta \in \bigwedge^{4}\left(V_{6} / v\right) \simeq\left(V_{6} / v\right)^{\vee}=v^{\perp} \subset V_{6}^{\vee}
$$

If $a$ is decomposable, that is, $a$ belongs to some $\bigwedge^{3} V_{3} \subset \Lambda^{3} V_{6}$, the hyperplanes $V_{5} \subset V_{6}$ such that $a \in v \wedge \wedge^{2} V_{5}$ satisfy $\wedge^{3} V_{3} \subset \wedge^{3} V_{5}$, hence $V_{3} \subset V_{5}$. This means that the natural map

$$
p^{\prime-1}(v) \rightarrow \mathbf{P}\left(A \cap\left(v \wedge \wedge^{2} V_{6}\right)\right)
$$




\section{O. Debarre and A. KuZnetsov}

is a $\mathbf{P}^{2}$-fibration over $\mathbf{P}\left(A \cap\left(v \wedge \wedge^{2} V_{6}\right)\right) \cap \operatorname{Gr}\left(3, V_{6}\right)$ and is an isomorphism over its complement. If $v \notin S$, the first case does not happen, hence

$$
p^{\prime-1}(v) \simeq \mathbf{P}\left(A \cap\left(v \wedge \wedge^{2} V_{6}\right)\right) .
$$

Since $\widehat{Y}_{A}^{\prime}$ can be defined as the zero-locus of a section of a rank 14 vector bundle on the smooth 18-dimensional variety $\mathbf{P}(A) \times \mathrm{FI}\left(1,5 ; V_{6}\right)$, any irreducible component of $\widehat{Y}_{A}^{\prime}$ has dimension at least 4. On the other hand, the description (B.6) of the fibers of $p^{\prime}$ outside of the surface $S$ implies $\operatorname{dim}\left(p^{-1}\left(Y_{A}^{1} \backslash S\right)\right)=4$, and, using also Theorem B.2,

$$
\begin{aligned}
\operatorname{dim}\left(p^{-1}\left(Y_{A}^{\geqslant 2} \backslash S\right)\right) & \leqslant \max \left\{\operatorname{dim}\left(p^{\prime-1}\left(Y_{A}^{2} \backslash S\right)\right), \operatorname{dim}\left(p^{-1}\left(Y_{A}^{3} \backslash S\right)\right), \operatorname{dim}\left(p^{\prime-1}\left(Y_{A}^{4} \backslash S\right)\right)\right\} \\
& \leqslant \max \{2+1,0+2,0+3\}=3 .
\end{aligned}
$$

Using the description of the fibers of $p^{\prime}$ over the surface $S$, we conclude that the irreducible components of $\widehat{Y}_{A}^{\prime}$ are

- a "main" component $\widehat{Y}_{A}^{\prime \prime}$ which dominates $Y_{A}$,

- one component $\{a\} \times \mathbf{P}\left(V_{3}\right) \times \mathbf{P}\left(V_{3}^{\perp}\right)$ for each decomposable vector $a \in \bigwedge^{3} V_{3} \subset A$.

It now remains to prove that $\widehat{Y}_{A}^{\prime \prime}$ defines the projective duality between $Y_{A}$ and $Y_{A^{\perp}}$. Let $v \in Y_{A}^{1} \backslash S$, and set $V_{5}:=q\left(p^{-1}(v)\right)$. Let us show that the hyperplane $\mathbf{P}\left(V_{5}\right) \subset \mathbf{P}\left(V_{6}\right)$ is tangent to $Y_{A}$ at $v$. Since $p^{\prime}$ is an isomorphism over a neighborhood of $v$, the tangent space $\mathbf{T}_{Y_{A}^{1}, v}$ is identified with the tangent space $\mathbf{T}_{\widehat{Y}_{A}^{\prime},\left(a, v, V_{5}\right)}$. Let $\left(a+a^{\prime} t, v+v^{\prime} t, V_{5}+V_{5}^{\prime} t\right)$ be a tangent vector to $\widehat{Y}_{A}^{\prime}$, that is, a $\mathbf{k}[t] / t^{2}$-point of this variety, with $a^{\prime} \in A$. Since $A$ is Lagrangian, we have

$$
a \wedge a^{\prime}=0 \text {. }
$$

On the other hand, by the definition of $\widehat{Y}_{A}^{\prime}$, we can write $a+a^{\prime} t=\left(v+v^{\prime} t\right) \wedge\left(\eta+\eta^{\prime} t\right)$, which gives $a=v \wedge \eta$ and $a^{\prime}=v \wedge \eta^{\prime}+v^{\prime} \wedge \eta$. Substituting into (B.7), we get

$$
0=(v \wedge \eta) \wedge\left(v \wedge \eta^{\prime}+v^{\prime} \wedge \eta\right)=v \wedge v^{\prime} \wedge \eta \wedge \eta
$$

But $v^{\prime}$ is the image of the tangent vector in $\mathbf{T}_{Y_{A}^{1}, v}$, while $v \wedge \eta \wedge \eta$ is the equation of $V_{5}$. Equation (B.8) therefore implies $v^{\prime} \in V_{5}$. Since this holds for any $v^{\prime}$, we deduce $\mathbf{T}_{Y_{A}^{1}, v} \subset \mathbf{P}\left(V_{5}\right)$. Since $v$ is a smooth point of the hypersurface $Y_{A}$, this implies $\mathbf{T}_{Y_{A}, v}=\mathbf{P}\left(V_{5}\right)$, as required.

Remark B.4. Assume that $A$ contains no decomposable vectors. First, the forgetful map $\widehat{Y}_{A}^{\prime} \rightarrow \widehat{Y}_{A}$ is an isomorphism: if $\left(a, v, V_{5}\right),\left(a^{\prime}, v, V_{5}\right) \in \widehat{Y}_{A}^{\prime}$ with $a \neq a^{\prime}$, there would be decomposable vectors in $A$ in the span of $a$ and $a^{\prime}$.

Second, the definition of $\widehat{Y}_{A}$ is symmetric with respect to $V_{6}$ and $V_{6}^{\vee}$. In particular, we obtain from (B.6) an identification $q^{-1}\left(V_{5}\right) \simeq \mathbf{P}\left(A \cap \bigwedge^{3} V_{5}\right)$, and $p$ takes $a \in A \cap \bigwedge^{3} V_{5}$ to the kernel of $a$ considered as a skew form on $V_{5}$ via the isomorphism $\bigwedge^{3} V_{5} \simeq \bigwedge^{2} V_{5}^{\vee}$.

The following two lemmas were used in this article.

Lemma B.5. If $A$ contains no decomposable vectors, we have

$$
\left(Y_{A}^{2} \times Y_{A^{\perp}}^{2}\right) \cap \mathrm{FI}\left(1,5 ; V_{6}\right) \not \subset(p \times q)\left(\widehat{Y}_{A}\right) .
$$

Proof. Assume, to the contrary, that we have $\left(Y_{A}^{\geqslant 2} \times Y_{A^{\perp}}^{\geqslant 2}\right) \cap \mathrm{FI}\left(1,5 ; V_{6}\right) \subset(p \times q)\left(\widehat{Y}_{A}\right)$. The left side is the intersection in $\mathbf{P}\left(V_{6}\right) \times \mathbf{P}\left(V_{6}^{\vee}\right)$ of the fourfold $Y_{A}^{\geqslant 2} \times Y_{A^{\perp}}^{\geqslant 2}$ with the hypersurface $\mathrm{FI}\left(1,5 ; V_{6}\right)$, hence has dimension everywhere at least 3 . On the other hand, it sits in $E:=p^{-1}\left(Y_{A}^{\geqslant 2}\right)$, the 


\section{Gushel-Mukai VARIETIES}

exceptional divisor of the birational morphism $p$, and it follows from the proof of Proposition B.3 that $E$ is irreducible of dimension 3 . Therefore, we have

$$
\left(Y_{A}^{\geqslant 2} \times Y_{A^{\perp}}^{\geqslant 2}\right) \cap \mathrm{FI}\left(1,5 ; V_{6}\right)=E .
$$

By symmetry, we also have

$$
\left(Y_{A}^{\geqslant 2} \times Y_{A^{\perp}}^{\geqslant 2}\right) \cap \mathrm{FI}\left(1,5 ; V_{6}\right)=q^{-1}\left(Y_{A^{\perp}}^{\geqslant 2}\right)=: E^{\prime},
$$

where the right side is the exceptional divisor of the birational morphism $q$. It follows that $E=E^{\prime}$.

Denote by $H$ and $H^{\prime}$ the respective restrictions to $Y_{A}$ and $Y_{A^{\perp}}$ of the hyperplane classes from $\mathbf{P}\left(V_{6}\right)$ and $\mathbf{P}\left(V_{6}^{\vee}\right)$. The birational isomorphism $q \circ p^{-1}: Y_{A} \rightarrow Y_{A \perp}$ is given by the Gauss map (that is, by the partial derivatives of the equation of $Y_{A}$ ). Since the equation of $Y_{A}$ has degree 6 and any point of $Y_{A}^{2}$ has multiplicity 2 on $Y_{A}$ [OGr12, Corollary 2.5], the partial derivatives have degree 5 and multiplicity 1 , and we get

$$
H^{\prime} \sim_{\operatorname{lin}} 5 H-E .
$$

By symmetry, we also have $H \sim_{\text {lin }} 5 H^{\prime}-E^{\prime}$. Combining these two equations, we obtain

$$
E^{\prime} \sim_{\operatorname{lin}} 24 H-5 E .
$$

From $E^{\prime}=E$, we obtain $24 H \sim_{\operatorname{lin}} 6 E$, which gives a contradiction, since $E$ has negative intersection with curves contracted by $p$ while $H$ has zero intersection with such curves.

Lemma B.6. If $A$ contains no decomposable vectors, the scheme $Y_{A}^{\geqslant 2} \subset \mathbf{P}\left(V_{6}\right)$ is not contained in a hyperplane.

Proof. We use the notation introduced in the proof of Lemma B.5. If $Y_{A}^{\geqslant 2}$ is contained in a hyperplane, the linear system $|H-E|$ is non-empty; hence the linear system

$$
\left|E^{\prime}\right|=|24 H-5 E|=|19 H+5(H-E)|
$$

is movable. But $E^{\prime}$ is the exceptional divisor of the birational morphism $q$; hence $E^{\prime}$ is rigid, so this gives a contradiction.

\section{B.3 Double EPW sextics}

The properties of EPW sextics that are of most interest come from the existence of a (finite) canonical double cover $f_{A}: \widetilde{Y}_{A} \rightarrow Y_{A}$ [OGr13, Section 1.2] with the following properties.

Theorem B.7 (O'Grady). Let $A \subset \wedge^{3} V_{6}$ be a Lagrangian subspace which contains no decomposable vectors, and let $Y_{A} \subset \mathbf{P}\left(V_{6}\right)$ be the associated EPW sextic.

(a) The double cover $f_{A}: \tilde{Y}_{A} \rightarrow Y_{A}$ is branched over the surface $Y_{A}^{\geqslant 2}$ and induces the universal cover of $Y_{A}^{1}$.

(b) The variety $\widetilde{Y}_{A}$ is irreducible and normal, and its singular locus is the finite set $f_{A}^{-1}\left(Y_{A}^{\geqslant 3}\right)$.

(c) When $Y_{A}^{\geqslant 3}$ is empty, $\widetilde{Y}_{A}$ is a smooth, hyperkähler fourfold which is a deformation of the symmetric square of a $K 3$ surface.

Proof. Item (a) is proved in [OGr13, Proof of Theorem 4.15, p. 179], item (b) follows from statement (3) in the introduction of [OGr13], and item (c) is [OGr06, Theorem 1.1(2)].

We draw a consequence of this theorem. 


\section{O. Debarre and A. Kuznetsov}

Proposition B.8. Let $A \subset \bigwedge^{3} V_{6}$ and $A^{\prime} \subset \bigwedge^{3} V_{6}^{\prime}$ be Lagrangian subspaces, and let $Y_{A} \subset \mathbf{P}\left(V_{6}\right)$ and $Y_{A^{\prime}} \subset \mathbf{P}\left(V_{6}^{\prime}\right)$ be the schemes defined in (B.1).

(a) Any linear isomorphism $\varphi: V_{6} \stackrel{\sim}{\longrightarrow} V_{6}^{\prime}$ such that $\left(\bigwedge^{3} \varphi\right)(A)=A^{\prime}$ induces an isomorphism $Y_{A} \stackrel{\sim}{\longrightarrow} Y_{A^{\prime}}$.

(b) Assume $Y_{A} \neq \mathbf{P}\left(V_{6}\right)$. Any isomorphism $Y_{A} \stackrel{\sim}{\longrightarrow} Y_{A^{\prime}}$ is induced by a linear isomorphism $\varphi: V_{6} \stackrel{\sim}{\longrightarrow} V_{6}^{\prime}$, and, if $A$ contains no decomposable vectors, $A^{\prime}$ contains no decomposable vectors and $\left(\bigwedge^{3} \varphi\right)(A)=A^{\prime}$.

Proof. Item (a) follows from the definition of $Y_{A}$.

(b) If $Y_{A} \neq \mathbf{P}\left(V_{6}\right)$, it follows from the Lefschetz theorem [Gro05, XII, corollaire 3.7] that $\operatorname{Pic}\left(Y_{A}\right)$ is generated by the hyperplane class. Any isomorphism $Y_{A} \stackrel{\sim}{\longrightarrow} Y_{A^{\prime}}$ is therefore induced by a linear isomorphism $\varphi: V_{6} \stackrel{\sim}{\longrightarrow} V_{6}^{\prime}$.

If $A$ contains no decomposable vectors, $\operatorname{Sing}\left(Y_{A}\right)$ is an integral surface of degree 40 (Theorem B.2), hence contains no planes, and the same holds for $\operatorname{Sing}\left(Y_{A^{\prime}}\right)$. It follows from (B.3) that $A^{\prime}$ contains no decomposable vectors. Applying Theorem B.2 again, we obtain $\varphi\left(Y_{A}^{1}\right)=Y_{A^{\prime}}^{1}$. This isomorphism lifts to the universal covers to an isomorphism $f_{A}^{-1}\left(Y_{A}^{1}\right) \stackrel{\sim}{\longrightarrow} f_{A^{\prime}}^{-1}\left(Y_{A^{\prime}}^{1}\right)$ and extends to the normal completions to an isomorphism $\widetilde{Y}_{A} \stackrel{\sim}{\longrightarrow} \widetilde{Y}_{A^{\prime}}$ (Theorem B.7). By [OGr16, Proof of Proposition 1.0.5], this implies $\left(\bigwedge^{3} \varphi\right)(A)=A^{\prime}$.

Finally, we characterize the automorphism group of an EPW sextic.

Proposition B.9 (O'Grady). Let $A \subset \bigwedge^{3} V_{6}$ be a Lagrangian subspace which contains no decomposable vectors, and let $Y_{A} \subset \mathbf{P}\left(V_{6}\right)$ be the associated EPW sextic.

(a) The automorphism group of $Y_{A}$ is finite and equal to the group

$$
\operatorname{PGL}\left(V_{6}\right)_{A}:=\left\{g \in \operatorname{PGL}\left(V_{6}\right) \mid\left(\bigwedge^{3} g\right)(A)=A\right\} .
$$

(b) When $A$ is very general, these groups are trivial.

Proof. (a) The equality $\operatorname{Aut}\left(Y_{A}\right)=\operatorname{PGL}\left(V_{6}\right)_{A}$ follows from Proposition B.8. These groups are finite because $A$ is a stable point of $\operatorname{LGr}\left(\bigwedge^{3} V_{6}\right)$ for the action of $\operatorname{PGL}\left(V_{6}\right)$ : the non-stable locus has 12 components, listed in [OGr16, Table 1], and one checks that they are all contained in the locus of Lagrangians which contain decomposable vectors.

(b) Let $f_{A}: \widetilde{Y}_{A} \rightarrow Y_{A}$ be the canonical double cover, where $\tilde{Y}_{A}$ is a smooth hyperkähler fourfold (Theorem B.7(c)). As explained in the proof of Proposition B.8(b), any automorphism $g$ of $Y_{A}$ is linear and lifts to an automorphism $\tilde{g}$ of $\widetilde{Y}_{A}$.

Let $h$ be the class in $H^{2}\left(\widetilde{Y}_{A}, \mathbf{Z}\right)$ of $f_{A}^{*}\left(\mathscr{O}_{Y_{A}}(1)\right)$. The automorphism $\tilde{g}$ fixes $h$, hence acts on its orthogonal $h^{\perp} \subset H^{2}\left(\widetilde{Y}_{A}, \mathbf{C}\right)$ for the Beauville-Bogomolov form as a linear map, which we denote by $\tilde{g}^{*}$. The line $H^{2,0}\left(\widetilde{Y}_{A}\right) \subset H^{2}\left(\widetilde{Y}_{A}, \mathbf{C}\right)$ is contained in an eigenspace of $\tilde{g}^{*}$, and the small deformations $A_{t}$ of $A$ for which $g$ extends must maintain $H^{2,0}\left(\widetilde{Y}_{A_{t}}\right)$ in that same eigenspace. It follows that if $g$ extends for all small deformations of $A$, the eigenspace containing $H^{2,0}\left(\widetilde{Y}_{A}\right)$ is all of $h^{\perp}$. Thus $\tilde{g}^{*}=\zeta \operatorname{Id}_{h^{\perp}}$, where $\zeta$ is some root of unity. Since $\tilde{g}^{*}$ is real, we have $\zeta= \pm 1$.

But the morphism $\operatorname{Aut}\left(\widetilde{Y}_{A}\right) \rightarrow \operatorname{Aut}\left(H^{2}\left(\widetilde{Y}_{A}, \mathbf{Z}\right)\right)$ is injective: its kernel is deformation invariant [HT13, Theorem 2.1], and it is trivial for symmetric squares of K3 surfaces [Bea83, Proposition 10]; the statement therefore follows from Theorem B.7(c). This implies that $\tilde{g}$ is either the identity or the involution associated with the double covering $f_{A}$. In both cases, $g=\operatorname{Id}_{Y_{A}}$. 


\section{Gushel-Mukai VARIETIES}

\section{B.4 EPW quartics}

We introduce certain quartic hypersurfaces in $\operatorname{Gr}\left(3, V_{6}\right)$ also associated with Lagrangian subspaces $A \subset \bigwedge^{3} V_{6}$. For each $\ell \geqslant 0$, we set

$$
Z_{A}^{\geqslant \ell}:=\left\{V_{3} \in \operatorname{Gr}\left(3, V_{6}\right) \mid \operatorname{dim}\left(A \cap\left(V_{6} \wedge \wedge^{2} V_{3}\right)\right) \geqslant \ell\right\} .
$$

The subschemes $Z_{A}^{\geqslant \ell} \subset \operatorname{Gr}\left(3, V_{6}\right)$ were recently studied in [IKKR17]. In particular, if $A$ contains no decomposable vectors, the scheme $Z_{A}=Z_{A}^{\geqslant 1}$ is an integral quartic hypersurface [IKKR17, Corollary 2.10]. We will call it the EPW quartic associated with $A$. We will only need the following duality property.

LEMmA B.10. The natural isomorphism $\operatorname{Gr}\left(3, V_{6}\right) \simeq \operatorname{Gr}\left(3, V_{6}^{\vee}\right)$ induces an identification between the EPW quartics $Z_{A} \subset \mathrm{Gr}\left(3, V_{6}\right)$ and $Z_{A^{\perp}} \subset \mathrm{Gr}\left(3, V_{6}^{\vee}\right)$.

Proof. The isomorphism of the Grassmannians takes $V_{3} \subset V_{6}$ to $V_{3}^{\perp} \subset V_{6}^{\vee}$. It is therefore enough to show that the isomorphism $\wedge^{3} V_{6} \rightarrow \wedge^{3} V_{6}^{\vee}$ given by the symplectic form takes the subspace $V_{6} \wedge \wedge^{2} V_{3}$ to the subspace $V_{6}^{\vee} \wedge \wedge^{2} V_{3}^{\perp}$. As both subspaces are Lagrangian, it is enough to verify that the pairing between $V_{6} \wedge \wedge^{2} V_{3}$ and $V_{6}^{\vee} \wedge \wedge^{2} V_{3}^{\perp}$ induced by the pairing between $V_{6}$ and $V_{6}^{\vee}$ is trivial, and this is straightforward.

\section{Appendix C. The projective duality of quadrics and Lagrangian subspaces}

Let $V$ be a $\mathbf{k}$-vector space. We discuss the projective duality of quadrics (of all possible dimensions) in $\mathbf{P}(V)$ and its interpretation in terms of Lagrangian geometry. To simplify the statements, it is convenient to define a quadric $Q$ in $\mathbf{P}(V)$ as a subvariety of a (possibly empty) linear subspace $\mathbf{P}(W) \subset \mathbf{P}(V)$ defined by a (possibly zero) quadratic form $q \in \mathrm{S}^{2} W^{\vee}$ (if $q=0$, then $Q=\mathbf{P}(W)$ is a linear subspace of $\mathbf{P}(V)$; it should not be confused with rank 1 quadrics, which are double linear subspaces). With each such form $q$, we can associate its kernel space $K \subset W$. The induced form on the (possibly zero) quotient space $W / K$ is then non-degenerate.

The projective dual variety $Q^{\vee} \subset \mathbf{P}\left(V^{\vee}\right)$ is also a quadric, which can be constructed as follows. The quadratic form $q$ induces an isomorphism

$$
q: W / K \stackrel{\sim}{\longrightarrow}(W / K)^{\vee}=K^{\perp} / W^{\perp},
$$

where $W^{\perp} \subset K^{\perp} \subset V^{\vee}$ are the orthogonals. The inverse isomorphism

$$
q^{-1}: K^{\perp} / W^{\perp} \stackrel{\sim}{\longrightarrow} W / K=\left(K^{\perp} / W^{\perp}\right)^{\vee}
$$

defines a quadratic form $q^{\vee}$ on the space $K^{\perp} \subset V^{\vee}$ with kernel $W^{\perp}$. The corresponding quadric in $\mathbf{P}\left(K^{\perp}\right) \subset \mathbf{P}\left(V^{\vee}\right)$ is the projective dual $Q^{\vee}$ of $Q$.

It is a classical observation that the projective duality of quadrics can be described in terms of Lagrangian subspaces in a symplectic vector space equipped with a Lagrangian direct sum decomposition. For the reader's convenience, we summarize this relation and develop it a bit.

We recall how a Lagrangian subspace gives a pair of projectively dual quadrics.

Proposition C.1. Let $(\mathbb{V}, \omega)$ be a symplectic $\mathbf{k}$-vector space, and let $\mathbb{L}_{1}$ and $\mathbb{L}_{2}$ be Lagrangian subspaces such that $\mathbb{V}=\mathbb{L}_{1} \oplus \mathbb{L}_{2}$. Denote by $\mathrm{pr}_{1}$ and $\mathrm{pr}_{2} \in \operatorname{End}(\mathbb{V})$ the projectors to $\mathbb{L}_{1}$ and $\mathbb{L}_{2}$.

(a) For any Lagrangian subspace $A \subset \mathbb{V}$, the bilinear form $q^{A}$ on $A$ defined by

$$
q^{A}(x, y):=\omega\left(\operatorname{pr}_{1}(x), \operatorname{pr}_{2}(y)\right)
$$




\section{O. Debarre And A. KuZnetsov}

is symmetric. Its kernel is given by

$$
\operatorname{Ker}\left(q^{A}\right)=\left(A \cap \mathbb{L}_{1}\right) \oplus\left(A \cap \mathbb{L}_{2}\right) .
$$

(b) The quadratic form $q^{A}$ induces quadratic forms $q_{1}^{A}$ and $q_{2}^{A}$ on the subspaces

$$
W_{1}:=\operatorname{pr}_{1}(A)=\left(A \cap \mathbb{L}_{2}\right)^{\perp} \subset \mathbb{L}_{1} \quad \text { and } \quad W_{2}:=\operatorname{pr}_{2}(A)=\left(A \cap \mathbb{L}_{1}\right)^{\perp} \subset \mathbb{L}_{2}
$$

with respective kernels

$$
K_{1}:=A \cap \mathbb{L}_{1} \subset W_{1} \quad \text { and } \quad K_{2}:=A \cap \mathbb{L}_{2} \subset W_{2} .
$$

The corresponding quadrics $Q_{1}^{A} \subset \mathbf{P}\left(W_{1}\right) \subset \mathbf{P}\left(\mathbb{L}_{1}\right)$ and $Q_{2}^{A} \subset \mathbf{P}\left(W_{2}\right) \subset \mathbf{P}\left(\mathbb{L}_{2}\right)$ are projectively dual with respect to the duality between $\mathbb{L}_{1}$ and $\mathbb{L}_{2}$ induced by the symplectic form $\omega$.

Proof. (a) For all $x, y \in A$, we have

$$
\begin{aligned}
q^{A}(x, y)-q^{A}(y, x) & =\omega\left(\operatorname{pr}_{1}(x), \operatorname{pr}_{2}(y)\right)+\omega\left(\operatorname{pr}_{2}(x), \operatorname{pr}_{1}(y)\right) \\
& =\omega\left(\operatorname{pr}_{1}(x)+\operatorname{pr}_{2}(x), \operatorname{pr}_{1}(y)+\operatorname{pr}_{2}(y)\right)-\omega\left(\operatorname{pr}_{1}(x), \operatorname{pr}_{1}(y)\right)-\omega\left(\operatorname{pr}_{2}(x), \operatorname{pr}_{2}(y)\right) .
\end{aligned}
$$

In this last expression, the first term equals $\omega(x, y)$, hence is zero since $A$ is Lagrangian; the second and the third terms are zero since $\mathbb{L}_{1}$ and $\mathbb{L}_{2}$ are Lagrangian. Thus the expression vanishes and $q^{A}$ is symmetric.

It remains to compute the kernel of $q^{A}$. Since $x=\operatorname{pr}_{1}(x)+\operatorname{pr}_{2}(x)$ and $\mathbb{L}_{2}$ is Lagrangian, we have

$$
q^{A}(x, y)=\omega\left(\operatorname{pr}_{1}(x), \operatorname{pr}_{2}(y)\right)=\omega\left(x-\operatorname{pr}_{2}(x), \operatorname{pr}_{2}(y)\right)=\omega\left(x, \operatorname{pr}_{2}(y)\right) .
$$

Since $A$ is Lagrangian, its $\omega$-orthogonal coincides with $A$, so $y \in \operatorname{Ker}\left(q^{A}\right)$ is equivalent to $\operatorname{pr}_{2}(y) \in A$. Writing $y=\operatorname{pr}_{1}(y)+\operatorname{pr}_{2}(y)$, this implies that also $\operatorname{pr}_{1}(y) \in A$. Thus $\operatorname{pr}_{1}(y) \in A \cap \mathbb{L}_{1}$ and $\operatorname{pr}_{2}(y) \in A \cap \mathbb{L}_{2}$. This proves one inclusion. For the other inclusion, both $A \cap \mathbb{L}_{1}$ and $A \cap \mathbb{L}_{2}$ are in the kernel of $q^{A}$ : for $A \cap \mathbb{L}_{1}$, this is because $\operatorname{pr}_{2}(y)=0$ for $y \in A \cap \mathbb{L}_{1}$, and for $A \cap \mathbb{L}_{2}$, it is because $\operatorname{pr}_{1}(x)=0$ for $x \in A \cap \mathbb{L}_{2}$.

(b) By (C.1), the kernels $A \cap \mathbb{L}_{2}$ and $A \cap \mathbb{L}_{1}$ of the restrictions $\left.\operatorname{pr}_{1}\right|_{A}$ and $\left.\mathrm{pr}_{2}\right|_{A}$ are contained in the kernel of $q^{A}$; hence $q^{A}$ induces quadratic forms on $W_{1}=\operatorname{pr}_{1}(A) \subset \mathbb{L}_{1}$ and $W_{2}=\operatorname{pr}_{2}(A) \subset \mathbb{L}_{2}$. The equalities $W_{1}=\left(A \cap \mathbb{L}_{2}\right)^{\perp}$ and $W_{2}=\left(A \cap \mathbb{L}_{1}\right)^{\perp}$ follow from the Lagrangian property of $A$. The kernels $K_{1}$ and $K_{2}$ of the induced quadratic forms $q_{1}^{A}$ and $q_{2}^{A}$ are the respective images of the kernel of $q^{A}$ under $\mathrm{pr}_{1}$ and $\mathrm{pr}_{2}$, so (C.2) implies $K_{1}=A \cap \mathbb{L}_{1}$ and $K_{2}=A \cap \mathbb{L}_{2}$.

Note that the subspace $A /\left(K_{1} \oplus K_{2}\right) \subset W_{1} / K_{1} \oplus W_{2} / K_{2}$ is the graph of an isomorphism $W_{1} / K_{1} \simeq W_{2} / K_{2}$. The duality between $W_{1} / K_{1}$ and $W_{2} / K_{2}$ given by the symplectic form $\omega$ identifies this isomorphism with the quadratic form $q_{1}^{A}$ and its inverse with $q_{2}^{A}$. This means that the quadrics $Q_{1}^{A}$ and $Q_{2}^{A}$ are projectively dual.

The following construction shows that any pair of projectively dual quadrics comes from a Lagrangian subspace.

Lemma C.2. Let $Q \subset \mathbf{P}(\mathbb{L})$ be a quadric, and let $Q^{\vee} \subset \mathbf{P}\left(\mathbb{L}^{\vee}\right)$ be its projective dual. In the space $\mathbb{V}:=\mathbb{L} \oplus \mathbb{L}^{\vee}$ endowed with the symplectic form

$$
\omega(x, y)=\left(x_{1}, y_{2}\right)-\left(y_{1}, x_{2}\right),
$$

there is a unique Lagrangian subspace $A \subset \mathbb{V}$ such that $Q_{1}^{A}=Q$ and $Q_{2}^{A}=Q^{\vee}$.

Proof. Let $K \subset \mathbb{L}$ and $W \subset \mathbb{L}$ be the kernel and span of $Q$, and let $q \in \mathrm{S}^{2} W^{\vee}$ be a quadratic form defining $Q$. Let $A$ be the kernel of the sum of the induced map $q: W \rightarrow W^{\vee}$ with the 


\section{Gushel-Mukai VARIETiES}

canonical surjection $\mathbb{L}^{\vee} \rightarrow W^{\vee}$. It fits into an exact sequence

$$
0 \rightarrow A \rightarrow W \oplus \mathbb{L}^{\vee} \rightarrow W^{\vee} \rightarrow 0 .
$$

The embedding $W \rightarrow \mathbb{L}$ induces an embedding $A \rightarrow \mathbb{L} \oplus \mathbb{L}^{\vee}=\mathbb{V}$. Let us show that the image is Lagrangian. It can be explicitly described as

$$
A=\left\{\left(x_{1}, x_{2}\right) \in \mathbb{L} \oplus \mathbb{L}^{\vee} \mid x_{1} \in W \text { and } x_{2} \equiv q\left(x_{1}\right) \bmod W^{\perp}\right\} .
$$

Given $x=\left(x_{1}, x_{2}\right)$ and $y=\left(y_{1}, y_{2}\right)$ in $A$, we have

$$
\omega(x, y)=\left(x_{1}, y_{2}\right)-\left(y_{1}, x_{2}\right)=\left(x_{1}, q\left(y_{1}\right)\right)-\left(y_{1}, q\left(x_{1}\right)\right)=q\left(y_{1}, x_{1}\right)-q\left(x_{1}, y_{1}\right)=0,
$$

so $A$ is indeed Lagrangian. Note that $\operatorname{pr}_{1}(A)=W$ by the definition of $A$. Moreover, if $q^{A}$ is the quadratic form on $A$ defined in Lemma C.1, for $x=\left(x_{1}, x_{2}\right)$ and $y=\left(y_{1}, y_{2}\right)$ in $A$, we have

$$
q^{A}(x, y)=\omega\left(\operatorname{pr}_{1}(x), \operatorname{pr}_{2}(y)\right)=\omega\left(x_{1}, y_{2}\right)=\left(x_{1}, q\left(y_{1}\right)\right)=q\left(x_{1}, y_{1}\right)=q\left(\operatorname{pr}_{1}(x), \operatorname{pr}_{1}(y)\right) .
$$

This shows $q_{1}^{A}=q$, so $Q_{1}^{A}=Q$. Since $Q_{2}^{A}$ is projectively dual to $Q_{1}^{A}$, we obtain $Q_{2}^{A}=Q^{\vee}$.

For the uniqueness, it is enough to check that the construction of this lemma is inverse to the construction of Proposition C.1, which is straightforward.

We now discuss the so-called "isotropic reduction" of Lagrangian subspaces.

Let $(\mathbb{V}, \omega)$ be a symplectic vector space. Let $\mathbb{I} \subset \mathbb{V}$ be an isotropic subspace, and let $\mathbb{I}^{\perp} \subset \mathbb{V}$ be its $\omega$-orthogonal. The restriction of the symplectic form $\omega$ to $\mathbb{I}^{\perp}$ is degenerate, and its kernel is $\mathbb{I} \subset \mathbb{I}^{\perp}$. Hence the form descends to a symplectic form $\bar{\omega}$ on $\overline{\mathbb{V}}:=\mathbb{I}^{\perp} / \mathbb{I}$. Moreover, for each Lagrangian subspace $\mathbb{L} \subset \mathbb{V}$, the subspace

$$
\overline{\mathbb{L}}:=\left(\mathbb{L} \cap \mathbb{I}^{\perp}\right) /(\mathbb{L} \cap \mathbb{I}) \subset \overline{\mathbb{V}}
$$

is Lagrangian. We call the symplectic space $\overline{\mathbb{V}}$ the isotropic reduction of $\mathbb{V}$ and the Lagrangian subspace $\overline{\mathbb{L}}$ the isotropic reduction of $\mathbb{L}$.

Let $\mathbb{V}=\mathbb{L}_{1} \oplus \mathbb{L}_{2}$ be a Lagrangian direct sum decomposition, and let $\mathbb{I} \subset \mathbb{L}_{1}$ (this is automatically isotropic). Isotropic reduction gives us a Lagrangian direct sum decomposition

$$
\overline{\mathbb{V}}=\overline{\mathbb{L}}_{1} \oplus \overline{\mathbb{L}}_{2},
$$

where $\overline{\mathbb{L}}_{1}=\mathbb{L}_{1} / \mathbb{I}$ and $\overline{\mathbb{L}}_{2}=\mathbb{L}_{2} \cap \mathbb{I}^{\perp}$. Furthermore, if $A \subset \mathbb{V}$ is Lagrangian, its isotropic reduction $\bar{A} \subset \overline{\mathbb{V}}$ produces a projectively dual pair of quadrics $\left(Q_{1}^{\bar{A}}, Q_{2}^{\bar{A}}\right)$. We will say that this pair is obtained from the pair $\left(Q_{1}^{A}, Q_{2}^{A}\right)$ by isotropic reduction with respect to $\mathbb{I}$. The next lemma gives a geometric relation between a pair of projectively dual quadrics and their isotropic reduction.

LEMma C.3. In the situation described above, we have the following:

(a) The quadratic form $q^{\bar{A}}$ on $\bar{A}$ is induced by the restriction of the quadratic form $q^{A}$ to $A \cap \mathbb{I}^{\perp} \subset$ A. In particular,

$$
Q^{A} \cap \mathbf{P}\left(A \cap \mathbb{I}^{\perp}\right)=\mathrm{C}_{A \cap \mathbb{I}}\left(Q^{\bar{A}}\right) .
$$

(b) The quadric $Q_{2}^{\bar{A}}$ is the linear section $Q_{2}^{A} \cap \mathbf{P}\left(\overline{\mathbb{L}}_{2}\right)$ of $Q_{2}^{A}$, and the quadric $Q_{1}^{\bar{A}}$ is its projective dual. Moreover, the span and the kernel of $Q_{2}^{\bar{A}}$ are

$$
\operatorname{Span}\left(Q_{2}^{\bar{A}}\right)=\left(\left(A \cap \mathbb{L}_{1}\right) /(A \cap \mathbb{I})\right)^{\perp} \subset \overline{\mathbb{L}}_{2}, \quad \operatorname{Ker}\left(Q_{2}^{\bar{A}}\right)=\left(A \cap\left(\mathbb{I} \oplus \overline{\mathbb{L}}_{2}\right)\right) /(A \cap \mathbb{I}) .
$$

Proof. (a) First, we have $A \cap \mathbb{I} \subset A \cap \mathbb{L}_{1} \subset \operatorname{Ker}\left(q^{A}\right)$. Thus $q^{A}$ induces a quadratic form on $\bar{A}$. Moreover, the projectors $\overline{\mathrm{pr}}_{1}$ and $\overline{\mathrm{pr}}_{2}$ onto the components of the decomposition $\overline{\mathbb{V}}=\overline{\mathbb{L}}_{1} \oplus \overline{\mathbb{L}}_{2}$ are 


\section{O. Debarre and A. Kuznetsov}

induced by the projectors $\mathrm{pr}_{1}$ and $\mathrm{pr}_{2}$. Hence, for $x, y \in A \cap \mathbb{I}^{\perp}$, we have

$$
q^{\bar{A}}(x, y)=\bar{\omega}\left(\overline{\operatorname{pr}}_{1}(x), \overline{\operatorname{pr}}_{2}(y)\right)=\omega\left(\operatorname{pr}_{1}(x), \operatorname{pr}_{2}(y)\right)=q^{A}(x, y) .
$$

This means that the quadratic form induced on $\bar{A}$ is $q^{\bar{A}}$ and thus proves the first equality.

(b) We prove that $Q_{2}^{\bar{A}}$ is a linear section of $Q_{2}^{A}$. Since $\mathbb{L}_{1} \subset \mathbb{I}^{\perp}$, we have

$$
\bar{W}_{2}:=\overline{\operatorname{pr}}_{2}(\bar{A})=\operatorname{pr}_{2}\left(A \cap \mathbb{I}^{\perp}\right)=\operatorname{pr}_{2}(A) \cap \operatorname{pr}_{2}\left(\mathbb{I}^{\perp}\right)=W_{2} \cap \overline{\mathbb{L}}_{2},
$$

and the kernel of the projection $A \cap \mathbb{I}^{\perp} \rightarrow \operatorname{pr}_{2}\left(A \cap \mathbb{I}^{\perp}\right)$ is $A \cap \mathbb{L}_{1}$. Therefore we have a commutative diagram

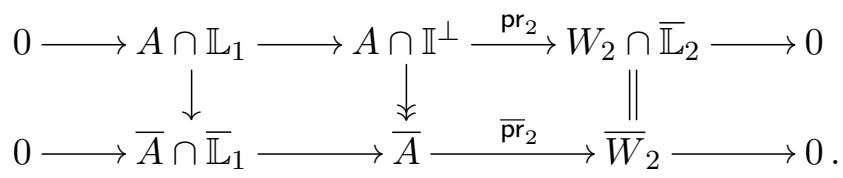

Since $A \cap \mathbb{L}_{1}$ is contained in the kernel of the quadratic form $\left.q^{A}\right|_{A \cap \mathbb{I} \perp}$, the quadratic form induced by it on $W_{2} \cap \overline{\mathbb{L}}_{2}$ via the projection $\mathrm{pr}_{2}$ coincides with the quadratic form obtained in two steps, by first inducing a quadratic form on $\bar{A}$ via the middle vertical arrow, and then on $\bar{W}_{2}$ via $\overline{\mathrm{pr}}_{2}$. The quadratic form induced via $\mathrm{pr}_{2}$ is $\left.q_{2}^{A}\right|_{W_{2} \cap \overline{\mathbb{L}}_{2}}$, the quadratic form induced via the vertical map is $q^{\bar{A}}$, and the quadratic form induced by it via $\overline{\mathrm{pr}}_{2}$ is $q_{2}^{\bar{A}}$. Therefore, by the commutativity of the right square, we deduce $\left.q_{2}^{A}\right|_{W_{2} \cap \overline{\mathbb{L}}_{2}}=q_{2}^{\bar{A}}$.

We now identify the span and the kernel of $Q_{2}^{\bar{A}}$. By Proposition C.1, the span is $\left(\bar{A} \cap \overline{\mathbb{L}}_{1}\right)^{\perp}$ and the kernel is $\bar{A} \cap \overline{\mathbb{L}}_{2}$. To describe the first, consider the commutative diagram above. Since the middle vertical map is surjective with kernel $A \cap \mathbb{I}$, the same is true for the left vertical map; hence $\bar{A} \cap \overline{\mathbb{L}}_{1}=\left(A \cap \mathbb{L}_{1}\right) /(A \cap \mathbb{I})$.

For the kernel, recall that $\bar{A}$ is the image of $A \cap \mathbb{I}^{\perp}$ under the projection $\mathbb{I}^{\perp} \rightarrow \overline{\mathbb{V}}$ with kernel $\mathbb{I}$. Therefore, $\bar{A} \cap \overline{\mathbb{L}}_{2}$ is the image in $\overline{\mathbb{V}}$ of the intersection of $A \cap \mathbb{I}^{\perp}$ with the preimage of $\overline{\mathbb{L}}_{2}$ in $\mathbb{I}^{\perp}$, that is, with $\mathbb{I} \oplus \overline{\mathbb{L}}_{2}$. Thus it is the image in $\overline{\mathbb{V}}$ of $A \cap\left(\mathbb{I} \oplus \overline{\mathbb{L}}_{2}\right)$. Since the kernel of the projection $\mathbb{I}^{\perp} \rightarrow \overline{\mathbb{V}}$ is $\mathbb{I}$, we see that $\bar{A} \cap \overline{\mathbb{L}}_{2}=\left(A \cap\left(\mathbb{I} \oplus \overline{\mathbb{L}}_{2}\right)\right) /(A \cap \mathbb{I})$.

Choosing an isotropic space appropriately, we can realize any subquadric in $Q_{2}$ as the result of an isotropic reduction applied to the pair $\left(Q_{1}, Q_{2}\right)$. This gives a convenient way to control the span and the kernel of a subquadric.

Corollary C.4. Let $\left(Q_{1}, Q_{2}\right)$ be a projectively dual pair of quadrics corresponding to a Lagrangian decomposition $\mathbb{V}=\mathbb{L}_{1} \oplus \mathbb{L}_{2}$ and a Lagrangian subspace $A \subset \mathbb{V}$. Let $\overline{\mathbb{L}}_{2} \subset \mathbb{L}_{2}$ be an arbitrary subspace, and set

$$
\mathbb{I}:=\mathbb{L}_{1} \cap \overline{\mathbb{L}}_{2}^{\perp} .
$$

The isotropic reduction with respect to $\mathbb{I}$ produces a projectively dual pair of quadrics $\left(\bar{Q}_{1}, \bar{Q}_{2}\right)$, where $\bar{Q}_{2}=Q_{2} \cap \mathbf{P}\left(\overline{\mathbb{L}}_{2}\right)$. In particular, the span and the kernel of $\bar{Q}_{2}$ are given by (C.4).

Proof. Since the pairing between $\mathbb{L}_{1}$ and $\mathbb{L}_{2}$ is non-degenerate and $\mathbb{I}$ is the orthogonal of $\overline{\mathbb{L}}_{2}$ in $\mathbb{L}_{1}$, it follows that $\overline{\mathbb{L}}_{2}$ is the orthogonal of $\mathbb{I}$ in $\mathbb{L}_{2}$, that is, $\overline{\mathbb{L}}_{2}=\mathbb{L}_{2} \cap \mathbb{I}^{\perp}$, and Lemma C.3 applies.

To conclude, we discuss a family version of the correspondence discussed in this appendix. Let $S$ be a scheme, let $\mathscr{V}$ be a vector bundle on $S$ equipped with a symplectic form $\omega: \bigwedge^{2} \mathscr{V} \rightarrow \mathscr{M}$ with values in a line bundle $\mathscr{M}$, and let $\mathscr{V}=\mathscr{L}_{1} \oplus \mathscr{L}_{2}$ be a Lagrangian direct sum decomposition. The latter induces an isomorphism

$$
\mathscr{L}_{2}^{\vee} \simeq \mathscr{L}_{1} \otimes \mathscr{M}^{-1}
$$




\section{Gushel-Mukai VARIETIES}

Let $\mathscr{A} \subset \mathscr{V}$ be another Lagrangian subbundle. Formula (C.1) then defines a quadratic form $q_{\mathscr{A}}: \mathscr{M}^{-1} \rightarrow \mathrm{S}^{2} \mathscr{A}^{\vee}$ on $\mathscr{A}$, but if we want to consider one of the two projectively dual quadrics, we should impose some constant-rank condition to ensure that the linear span of the quadric in question is a vector bundle.

Lemma C.5. Assume that the morphism $\mathscr{A} \hookrightarrow \mathscr{V} \stackrel{\mathrm{pr}_{2}}{\longrightarrow} \mathscr{L}_{2}$ has constant rank, and let $\mathscr{W}_{2} \subset \mathscr{L}_{2}$ be its image. Then the family of quadrics $q_{\mathscr{A}}$ induces a family $q_{\mathscr{A}, 2}: \mathscr{M}^{-1} \rightarrow \mathrm{S}^{2} \mathscr{W}_{2}^{\vee}$ of quadrics on $\mathscr{W}_{2}$, and there are isomorphisms of sheaves

$$
\begin{aligned}
\operatorname{Coker}\left(\mathscr{W}_{2} \otimes \mathscr{M}^{-1} \stackrel{q_{\mathscr{A}, 2}}{\longrightarrow} \mathscr{W}_{2}^{\vee}\right) & \simeq \operatorname{Coker}\left(\mathscr{A} \otimes \mathscr{M}^{-1} \stackrel{\mathrm{pr}_{1}}{\longrightarrow} \mathscr{L}_{2}^{\vee}\right) \\
& \simeq \operatorname{Coker}\left(\mathscr{L}_{2} \otimes \mathscr{M}^{-1} \stackrel{\mathrm{pr}_{1}^{\vee}}{\longrightarrow} \mathscr{A}^{\vee}\right)
\end{aligned}
$$

and similarly for the kernels.

Proof. By the definition of $\mathscr{W}_{2}$, there are epimorphisms $\mathscr{A} \rightarrow \mathscr{W}_{2}$ and $\mathscr{L}_{1} \otimes \mathscr{M}^{-1} \simeq \mathscr{L}_{2}^{\vee} \rightarrow \mathscr{W}_{2}^{\vee}$. They combine into a commutative diagram

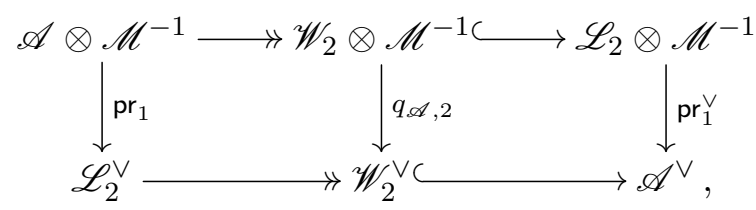

where the kernels and cokernels of the horizontal compositions are identified by the maps $\operatorname{pr}_{1}$ and $\operatorname{pr}_{1}^{\vee}$ (the kernels also can be characterized as the intersection of the subbundles $\mathscr{A}$ and $\mathscr{L}_{1}$ inside $\mathscr{V}$, twisted by $\mathscr{M}^{-1}$, and the cokernels are dual to the kernels, up to a twist). It follows that the cokernels of the vertical maps $\mathrm{pr}_{1}, q_{\mathscr{A}, 2}$, and $\mathrm{pr}_{1}^{\vee}$ are isomorphic, whence the lemma.

We also discuss a family version of isotropic reduction. Assume that in addition to the above, we are given a vector subbundle $\mathscr{I} \subset \mathscr{L}_{1}$. The vector bundle $\overline{\mathscr{V}}$ defined as the cohomology of the monad $\mathscr{I} \hookrightarrow \mathscr{V} \rightarrow \mathscr{I}^{\vee} \otimes \mathscr{M}$ then has a natural symplectic structure, and if $\mathscr{L} \subset \mathscr{V}$ is a Lagrangian subbundle such that the composition $\mathscr{L} \hookrightarrow \mathscr{V} \rightarrow \mathscr{I} \vee \otimes \mathscr{M}$ has constant rank, the sheaf $\overline{\mathscr{L}}:=\operatorname{Ker}\left(\mathscr{L} \rightarrow \mathscr{I}^{\vee} \otimes \mathscr{M}\right) / \operatorname{Ker}\left(\mathscr{I} \rightarrow \mathscr{L}^{\vee} \otimes \mathscr{M}\right)$ is locally free and is a Lagrangian subbundle in $\overline{\mathscr{V}}$. In particular, when applied to $\mathscr{L}=\mathscr{L}_{1}$ and $\mathscr{L}=\mathscr{L}_{2}$, we get

$$
\overline{\mathscr{L}}_{1}=\mathscr{L}_{1} / \mathscr{I} \quad \text { and } \quad \overline{\mathscr{L}}_{2}=\operatorname{Ker}\left(\mathscr{L}_{2} \rightarrow \mathscr{I}^{\vee} \otimes \mathscr{M}\right) \text {. }
$$

Lemma C.6. One has a Lagrangian direct sum decomposition $\overline{\mathscr{V}}=\overline{\mathscr{L}}_{1} \oplus \overline{\mathscr{L}}_{2}$. Moreover, if $\mathscr{A} \subset \mathscr{V}$ is a Lagrangian subbundle such that the composition $\mathscr{A} \rightarrow \mathscr{V} \rightarrow \mathscr{I}^{\vee} \otimes \mathscr{M}$ has constant rank, the vector bundle $\overline{\mathscr{W}}_{2}$ is a subbundle in $\mathscr{W}_{2}$ and the quadratic form $q_{\mathscr{A}_{2}}$ on $\overline{\mathscr{W}}_{2}$ equals the restriction of the quadratic form $q_{\mathscr{A}, 2}$. Finally, the subbundle $\widetilde{\mathscr{L}}_{2}:=\mathscr{I} \oplus \overline{\mathscr{L}}_{2} \subset \mathscr{L}_{1} \oplus \mathscr{L}_{2}=\mathscr{V}$ is Lagrangian, and there is an exact sequence

$0 \rightarrow \operatorname{Coker}\left(\overline{\mathscr{W}}_{2} \otimes \mathscr{M}^{-1} \rightarrow \overline{\mathscr{W}}_{2}^{\vee}\right) \rightarrow \operatorname{Coker}\left(\mathscr{A} \otimes \mathscr{M}^{-1} \rightarrow \widetilde{\mathscr{L}}_{2}^{\vee}\right) \rightarrow \operatorname{Coker}\left(\mathscr{A} \otimes \mathscr{M}^{-1} \rightarrow \mathscr{I}^{\vee}\right) \rightarrow 0$.

In particular, if the map $\mathscr{A} \rightarrow \mathscr{I}^{\vee} \otimes \mathscr{M}$ is an epimorphism, there are isomorphisms

$$
\operatorname{Coker}\left(\overline{\mathscr{W}}_{2} \otimes \mathscr{M}^{-1} \rightarrow \overline{\mathscr{W}}_{2}^{\vee}\right) \simeq \operatorname{Coker}\left(\mathscr{A} \otimes \mathscr{M}^{-1} \rightarrow \widetilde{\mathscr{L}}_{2}^{\vee}\right) \simeq \operatorname{Coker}\left(\widetilde{\mathscr{L}}_{2} \otimes \mathscr{M}^{-1} \rightarrow \mathscr{A}^{\vee}\right)
$$

Proof. Just repeat the proof of Lemma C.3.

\section{ACKNOWLEDGEMENTS}

This article owns much to the ideas and work of Kieran O'Grady, Atanas Iliev, and Laurent 


\section{O. Debarre and A. Kuznetsov}

Manivel. We would also like to thank Olivier Benoist, Alex Perry, and Yuri Prokhorov for their help and many interesting discussions, and the referee for useful comments.

\section{REFERENCES}

ACGH85 E. Arbarello, M. Cornalba, P. A. Griffiths, and J. Harris, Geometry of algebraic curves, Vol. I, Grundlehren math. Wiss., vol. 267 (Springer-Verlag, New York, 1985); https://doi.org/10. 1007/978-1-4757-5323-3.

AH81 E. Arbarello and J. Harris, Canonical curves and quadrics of rank 4, Compos. Math. 43 (1981), no. 2, 145-179; http://www.numdam.org/item?id=CM_1981_44_2_145_0.

Ale91 V. A. Alexeev, Theorems about good divisors on log Fano varieties (case of index $r>n-2$ ), Algebraic Geometry (Chicago, IL, 1989), Lecture Notes in Math., vol. 1479 (Springer, Berlin, 1991), 1-9; https://doi.org/10.1007/BFb0086258.

Bea83 A. Beauville, Some remarks on Kähler manifolds with $c_{1}=0$, Classification of Algebraic and Analytic Manifolds (Katata, 1982), Progr. Math., vol. 39 (Birkhäuser Boston, Boston, MA, 1983), 1-26; https://doi.org/10.1007/BF02592068.

DIM12 O. Debarre, A. Iliev, and L. Manivel, On the period map for prime Fano threefolds of degree 10, J. Algebraic Geom. 21 (2012), no. 1, 21-59; https://doi.org/10.1090/ S1056-3911-2011-00594-8.

DIM15_ Special prime Fano fourfolds of degree 10 and index 2, Recent Advances in Algebraic Geometry, London Math. Soc. Lecture Note Ser., vol. 417, eds C. Hacon, M. Mustaţă, and M. Popa (Cambridge Univ. Press, Cambridge, 2015), 123-155.

DK16 O. Debarre and A. Kuznetsov, Gushel-Mukai varieties: linear spaces and periods, Kyoto J. Math., to appear, arXiv:1605.05648.

Dol12 I. V. Dolgachev, Classical algebraic geometry. A modern view (Cambridge Univ. Press, Cambridge, 2012); https://doi.org/10.1017/CB09781139084437.

EPW01 D. Eisenbud, S. Popescu, and C. Walter, Lagrangian subbundles and codimension 3 subcanonical subschemes, Duke Math. J. 107 (2001), no. 3, 427-467; https://doi.org/10.1215/ S0012-7094-01-10731-X.

GL87 M. Green and R. Lazarsfeld, Special divisors on curves on a K3 surface, Invent. Math. 89 (1987), no. 2, 357-370; https://doi.org/10.1007/BF01389083.

GLT15 F. Greer, Z. Li, and Z. Tian, Picard groups on moduli of K3 surfaces with Mukai models, Int. Math. Res. Not. 2015 (2015), no. 16, 7238-7257; https://doi.org/10.1093/imrn/rnu152.

Gro05 A. Grothendieck, Cohomologie locale des faisceaux cohérents et théorèmes de Lefschetz locaux et globaux (SGA 2), Séminaire de Géométrie Algébrique du Bois Marie, 1962, Revised reprint of the 1968 original, Doc. Math. (Paris), vol. 4 (Soc. Math. France, Paris, 2005).

Gus83 N.P. Gushel', On Fano varieties of genus 6, Izv. Math. 21 (1983), no. 3, 445-459; https: //doi.org/10.1070/IM1983v021n03ABEH001801.

HT13 B. Hassett and Yu. Tschinkel, Hodge theory and Lagrangian planes on generalized Kummer fourfolds, Mosc. Math. J. 13 (2013), no. 1, 33-56; http://www.mathjournals.org/mmj/ 2013-013-001/2013-013-001-003.html.

IKKR17 A. Iliev, G. Kapustka, M. Kapustka, and K. Ranestad, EPW cubes, J. reine angew. Math., published online on 11 August 2016, https://doi.org/10.1515/crelle-2016-0044, to appear in print.

IM11 A. Iliev and L. Manivel, Fano manifolds of degree ten and EPW sextics, Ann. Sci. Éc. Norm. Supér. (4) 44 (2011), no. 3, 393-426; http://www.numdam.org/item?id=ASENS_2011_4_44_ 3_393_0.

IP99 V. A. Iskovskikh and Yu. G. Prokhorov, Fano varieties, in AlgebraicGometry, V, Encyclopaedia Math. Sci., vol. 47, eds A. Parshin and I. Shafarevich (Springer, Berlin, 1999), 1-247. 


\section{Gushel-Mukai varieties}

Isk77 V. A. Iskovskih, Fano 3-folds. I, Math. USSR Izv. 11 (1977), no. 3, 485-527; https://doi. org/10.1070/IM1977v011n03ABEH001733.

JK04 T. Johnsen and A. L. Knutsen, K3 projective models in scrolls, Lecture Notes in Math., vol. 1842 (Springer-Verlag, Berlin, 2004); https://doi.org/10.1007/b97183.

KP16 A. Kuznetsov and A. Perry, Derived categories of Gushel-Mukai varieties, 2016, arXiv:1605.06568.

Kuz16 A. Kuznetsov, Küchle fivefolds of type c5, Math. Z. 284 (2016), no. 3-4, 1245-1278; https: //doi.org/10.1007/s00209-016-1707-9.

Mel99 M. Mella, Existence of good divisors on Mukai varieties, J. Algebraic Geom. 8 (1999), no. 2, 197-206.

MM83 S. Mori and S. Mukai, On Fano 3-folds with $B_{2} \geqslant 2$, Algebraic Varieties and Analytic Varieties (Tokyo, 1981), Adv. Stud. Pure Math., vol. 1 (North-Holland, Amsterdam, 1983), 101-129.

Muk89 S. Mukai, Biregular classification of Fano 3-folds and Fano manifolds of coindex 3, Proc. Nat. Acad. Sci. USA 86 (1989), no. 9, 3000-3002; https://doi.org/10.1073/pnas.86.9.3000.

Muk93_, Curves and Grassmannians, Algebraic Geometry and Related Topics (Inchon, 1992), Conf. Proc. Lecture Notes Algebraic Geom., I (Int. Press, Cambridge, MA, 1993), 19-40.

Muk02 New development of theory of Fano 3-folds: vector bundle method and moduli problem, Sugaku Expositions 11 (2002), no. 2, 125-150.

Mum70 D. Mumford, Varieties defined by quadratic equations, Questions on Algebraic Varieties (C.I.M.E., III Ciclo, Varenna, 1969) (Edizioni Cremonese, Rome, 1970), 29-100.

OGr06 K. G. O'Grady, Irreducible symplectic 4-folds and Eisenbud-Popescu-Walter sextics, Duke Math. J. 134 (2006), no. 1, 99-137; https://doi.org/10.1215/S0012-7094-06-13413-0. Extended version available at arXiv:math/0507398,

OGr08a , Dual double EPW-sextics and their periods, Pure Appl. Math. Q. 4 (2008), no. 2, 427-468; https://doi.org/10.4310/PAMQ.2008.v4.n2.a6.

OGr08b Irreducible symplectic 4-folds numerically equivalent to (K3) ${ }^{[2]}$, Commun. Contemp. Math. 10 (2008), no. 4, 553-608; https://doi.org/10.1142/S0219199708002909.

OGr12 , EPW-sextics: taxonomy, Manuscripta Math. 138 (2012), no. 1-2, 221-272; https: //doi.org/10.1007/s00229-011-0472-7.

OGr13 D Double covers of EPW-sextics, Michigan Math. J. 62 (2013), no. 1, 143-184; https: //doi.org/10.1307/mmj/1363958245.

OGr15_ Periods of double EPW-sextics, Math. Z. 280 (2015), no. 1-2, 485-524; https://doi. org/10.1007/s00209-015-1434-7.

OGr16_ Moduli of double EPW-sextics, Mem. Amer. Math. Soc. 240 (2016), no. 1136; https: //doi.org/10.1090/memo/1136.

PCS05 V.V. Przhyjalkowski, I. A. Cheltsov, and K. A. Shramov, Hyperelliptic and trigonal Fano threefolds, Izv. Math. 69 (2005), no. 2, 365-421; https://doi.org/10.1070/ IM2005v069n02ABEH000533.

PvdV99 J. Piontkowski and A. van de Ven, The automorphism group of linear sections of the Grassmannians $\mathbf{G}(1, N)$, Doc. Math. 4 (1999), 623-664; https://www.math.uni-bielefeld.de/ documenta/vol-04/19.html.

Rot49 L. Roth, Algebraic varieties with canonical curve sections, Ann. Mat. Pura Appl. 29 (1949), 91-97; https://doi.org/10.1007/BF02413916.

RS06 G.V. Ravindra and V. Srinivas, The Grothendieck-Lefschetz theorem for normal projective varieties, J. Algebraic Geom. 15 (2006), no. 3, 563-590; https://doi.org/10.1090/ S1056-3911-05-00421-2.

She89 N. I. Shepherd-Barron, Invariant theory for $S_{5}$ and the rationality of $M_{6}$, Compos. Math. 70 (1989), no. 1, 13-25; http://www.numdam.org/item?id=CM_1989_-70_1_13_0. 


\section{O. Debarre and A. Kuznetsov}

She92 The rationality of quintic Del Pezzo surfaces - a short proof, Bull. London Math. Soc. 24 (1992), no. 3, 249-250; https://doi.org/10.1112/blms/24.3.249.

Wey03 J. Weyman, Cohomology of vector bundles and syzygies, Cambridge Tracts in Math., vol. 149 (Cambridge Univ. Press, Cambridge, 2003); https://doi.org/10.1017/CB09780511546556.

Olivier Debarre olivier.debarre@ens.fr

Université Paris Diderot, École normale supérieure, PSL Research University, CNRS, Département Mathématiques et Applications, 45 rue d'Ulm, 75230 Paris cedex 05, France

Alexander Kuznetsov akuznet@mi.ras.ru

Algebraic Geometry Section, Steklov Mathematical Institute, 8 Gubkin Str., Moscow, 119991 Russia

The Poncelet Laboratory, Independent University of Moscow, 11 Bolshoy Vlasyevskiy Pereulok, Moscow, 19902 Russia

Laboratory of Algebraic Geometry, National Research University Higher School of Economics, 6 Usacheva Str., Moscow, 119048 Russia 